\title{
2017 ESC focused update on dual antiplatelet therapy in coronary artery disease developed in collaboration with EACTS
}

\section{The Task Force for dual antiplatelet therapy in coronary artery disease of the European Society of Cardiology (ESC) and of the European Association for Cardio-Thoracic Surgery (EACTS)}

\author{
Authors/Task Force Members: Marco Valgimigli* (Chairperson) (Switzerland), \\ Héctor Bueno (Spain), Robert A. Byrne (Germany), Jean-Philippe Collet (France), \\ Francesco Costa (Italy), Anders Jeppsson ${ }^{1}$ (Sweden), Peter Jüni (Canada), \\ Adnan Kastrati (Germany), Philippe Kolh (Belgium), Laura Mauri (USA), \\ Gilles Montalescot (France), Franz-Josef Neumann (Germany), \\ Mate Petricevic ${ }^{1}$ (Croatia), Marco Roffi (Switzerland), Philippe Gabriel Steg \\ (France), Stephan Windecker (Switzerland), and Jose Luis Zamorano (Spain)
}

Additional Contributor: Glenn N. Levine (USA)

Document Reviewers: Lina Badimon (CPG Review Coordinator) (Spain), Pascal Vranckx (CPG Review Coordinator) (Belgium), Stefan Agewall (Norway), Felicita Andreotti (Italy), Elliott Antman (USA), Emanuele Barbato (Italy), Jean-Pierre Bassand (France), Raffaele Bugiardini (Italy), Mustafa Cikirikcioglu' (Switzerland), Thomas Cuisset (France), Michele De Bonis (Italy), Victora Delgado (The Netherlands), Donna Fitzsimons (UK), Oliver Gaemperli (Switzerland), Nazzareno Galiè (Italy), Martine Gilard (France),

* Corresponding author: Marco Valgimigli, Cardiology, Inselspital, Freiburgstrasse 8, 3010 Bern, Switzerland. Tel: +4131632 3077, Fax: +41 10 7035258, E-mail: marco.valgimigli@insel.ch. ESC Committee for Practice Guidelines (CPG) and National Cardiac Societies document reviewers: listed in the Appendix.

${ }^{1}$ Representing the EACTS

ESC entities having participated in the development of this document:

Associations: Acute Cardiovascular Care Association (ACCA), European Association of Preventive Cardiology (EAPC), European Association of Percutaneous Cardiovascular Interventions (EAPCI).

Working Groups: Cardiovascular Pharmacotherapy, Cardiovascular Surgery, Coronary Pathophysiology and Microcirculation, Peripheral Circulation, Pulmonary Circulation and Right Ventricular Function, Thrombosis, Valvular Heart Disease.

The content of these European Society of Cardiology (ESC) Guidelines has been published for personal and educational use only. No commercial use is authorized. No part of the ESC Guidelines may be translated or reproduced in any form without written permission from the ESC. Permission can be obtained upon submission of a written request to Oxford University Press, the publisher of the European Heart Journal and the party authorized to handle such permissions on behalf of the ESC (journals.permissions@oxfordjournals.org).

Disclaimer. The ESC Guidelines represent the views of the ESC and were produced after careful consideration of the scientific and medical knowledge and the evidence available at the time of their publication. The ESC is not responsible in the event of any contradiction, discrepancy and/or ambiguity between the ESC Guidelines and any other official recommendations or guidelines issued by the relevant public health authorities, in particular in relation to good use of healthcare or therapeutic strategies. Health professionals are encouraged to take the ESC Guidelines fully into account when exercising their clinical judgment, as well as in the determination and the implementation of preventive, diagnostic or therapeutic medical strategies; however, the ESC Guidelines do not override, in any way whatsoever, the individual responsibility of health professionals to make appropriate and accurate decisions in consideration of each patient's health condition and in consultation with that patient and, where appropriate and/or necessary, the patient's caregiver. Nor do the ESC Guidelines exempt health professionals from taking into full and careful consideration the relevant official updated recommendations or guidelines issued by the competent public health authorities, in order to manage each patient's case in light of the scientifically accepted data pursuant to their respective ethical and professional obligations. It is also the health professional's responsibility to verify the applicable rules and regulations relating to drugs and medical devices at the time of prescription.

The article has been co-published with permission in the European Heart Journal [DOI: 10.1093/eurheartj/ehx419] on behalf of the European Society of Cardiology and European Journal of Cardio-Thoracic Surgery [DOI 10.1093/ejcts/ezx334] on behalf of the European Association for Cardio-Thoracic Surgery. All rights reserved in respect of European Heart Journal, (c) European Society of Cardiology 2017. The articles are identical except for minor stylistic and spelling differences in keeping with each journal's style. Either citation can be used when citing this article.

For permissions, please email journals.permissions@oup.com. 
Christian W. Hamm (Germany), Borja Ibanez (Spain), Bernard lung (France), Stefan James (Sweden), Juhani Knuuti (Finland), UIf Landmesser (Germany), Christophe Leclercq (France), Maddalena Lettino (Italy), Gregory Lip (UK), Massimo Francesco Piepoli (Italy), Luc Pierard (Belgium), Markus Schwerzmann (Switzerland), Udo Sechtem (Germany), lain A. Simpson (UK), Miguel Sousa Uva' (Portugal), Eugenio Stabile (Italy), Robert F. Storey (UK), Michal Tendera (Poland), Frans Van de Werf (Belgium), Freek Verheugt (The Netherlands), and Victor Aboyans (CPG Supervisor) (France)

The disclosure forms of all experts involved in the development of this focused update are available on the ESC website http://www.escardio.org/guidelines.

The Addenda and Clinical Cases companion document of this focused update are available at: www.escardio. org/Guidelines/Clinical-Practice-Guidelines/20I7-focused-update-on-dual-antiplatelet-therapy-dapt Web addenda

Online publish-ahead-of-print 26 August 2017

Keywords Guidelines - Aspirin • Clopidogrel • Ticagrelor • Prasugrel • Dual antiplatelet therapy • Acute coronary syndromes - Coronary artery bypass grafting - Coronary artery disease • Drug-eluting stents • Myocardial infarction - Stent thrombosis • Bleeding - Percutaneous coronary intervention - Recommendation - Revascularization - Risk stratification - Stents - Stable angina • Stable coronary artery disease • Oral anticoagulant • Triple therapy • DAPT score • PRECISE-DAPT score • Non-cardiac surgery

\section{Table of Contents}

Abbreviations and acronyms...........................215

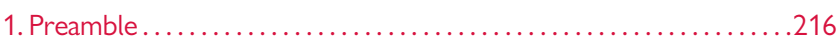

2. Introduction ............................................ 218

2.1 Short- and long-term outcomes after percutaneous coronary

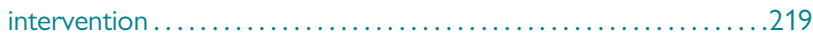

2.2 Risk of stent thrombosis in relation to stent type............ 219

2.3 Short- and long-term outcomes after coronary artery bypass

surgery.

2.4 Short- and long-term outcomes after medically managed acute coronary syndrome.

3. Efficacy and safety of dual antiplatelet therapy and risk

stratification tools

3.1 Dual antiplatelet therapy for the prevention of stent

thrombosis.....

3.2 Dual antiplatelet therapy for the prevention of spontaneous

myocardial infarction..................................219

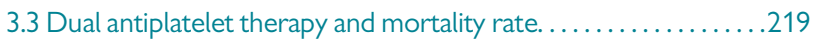

3.4 Safety of dual antiplatelet therapy .....................219

3.5 Risk stratification tools for ischaemia and bleeding risks .......219

3.6 Type of $\mathrm{P}_{2} \mathrm{Y}_{12}$ inhibitor and timing of initiation ..............221

3.7 Measures to minimize bleeding while on dual

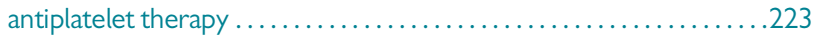

3.8 Switching between oral $\mathrm{P}_{2} \mathrm{Y}_{12}$ inhibitors. ...............225

4. Dual antiplatelet therapy and percutaneous coronary

intervention. ...

4.1 Dual antiplatelet therapy after percutaneous coronary

intervention for stable coronary artery disease.

4.2 Dual antiplatelet therapy after percutaneous coronary

intervention for acute coronary syndrome....................231

4.3 Gaps in the evidence

.233
5. Dual antiplatelet therapy and cardiac surgery ................234

5.1 Dual antiplatelet therapy in patients treated with coronary artery bypass surgery for stable coronary artery disease .........234

5.2 Dual antiplatelet therapy in patients treated with coronary

artery bypass surgery for acute coronary syndrome . ............234

5.3 Dual antiplatelet therapy for prevention of graft occlusion. . ... .237

5.4 Gaps in the evidence .............................237

6. Dual antiplatelet therapy for patients with medically managed

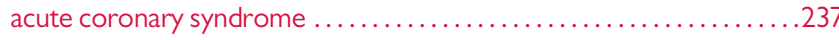

7. Dual antiplatelet therapy for patients with indication for oral anticoagulation.....................................238

7.1 Risk stratification and strategies to improve outcome after percutaneous coronary intervention. ......................238

7.2 Duration of triple therapy .............................239

7.3 Cessation of all antiplatelet agents.........................242

7.4 Type of anticoagulants..................................242

7.5 Type of stent. .................................... 242

8. Elective non-cardiac surgery in patients on dual antiplatelet

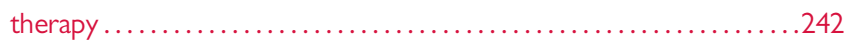

9. Gender consideration and special populations..................245

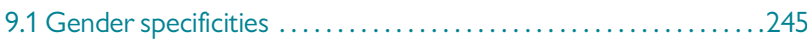

9.2 Diabetes mellitus................................. 245

9.3 Lower-extremities artery disease .......................245

9.4 Complex percutaneous coronary intervention ..............248

9.5 Dual antiplatelet therapy decision making in patients with

stent thrombosis . ....................................... 248

9.6 Patients who develop bleeding while on treatment. ............248

10. Key messages. ..........................................249

11. Evidenced-based 'to do and not to do' messages .................249

12. Web addenda and Clinical Cases companion document ..........251

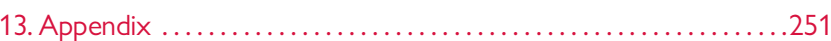

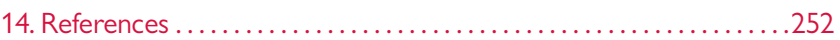




\section{Abbreviations and acronyms}

$A B C$

ACCA

ACCOAST

\section{ACS}

ADP

AF

ANTARCTIC

ARCTIC-Interruption

ART

ASA

ATACAS

ATLANTIC

BARC

b.i.d

BMS

CABG

CAD

$\mathrm{CHADS}_{2}$

$\mathrm{CHA}_{2} \mathrm{DS}_{2}-\mathrm{VASC}$

CHARISMA

$\mathrm{Cl}$

COGENT

CORONARY

CPG

$\mathrm{CrCl}$

CREDO

CRUSADE
Age, Biomarkers (GDF-15, cTnT-hs, and haemoglobin), and Clinical history (previous bleeding)

Acute Cardiovascular Care Association

A Comparison of Prasugrel at the Time of Percutaneous Coronary Intervention Or as Pretreatment At the Time of Diagnosis in Patients with Non-ST-Elevation

Myocardial Infarction

Acute coronary syndrome

Adenosine 5'-diphosphate

Atrial fibrillation

Platelet Function Monitoring to Adjust Antiplatelet Therapy in Elderly Patients Stented for an Acute Coronary Syndrome Assessment by a Double Randomisation of a Conventional Antiplatelet Strategy Versus a Monitoring-Guided Strategy for DrugEluting Stent Implantation and, of Treatment Interruption Versus Continuation 1 Year After Stenting-Interruption

Arterial Revascularisation Trial

Acetylsalicylic acid

Aspirin and Tranexamic Acid for Coronary Artery Surgery

Administration of Ticagrelor in the Cath Lab or in the Ambulance for New ST Elevation Myocardial Infarction to Open the Coronary Artery

Bleeding Academic Research Consortium Bis in die (twice a day)

Bare-metal stent

Coronary artery bypass graft surgery

Coronary artery disease

Cardiac failure, Hypertension, Age,

Diabetes, Stroke (Doubled)

Cardiac failure, Hypertension, Age $\geq 75$ (2 points), Diabetes, Stroke (2 points)-Vascular disease, Age 65-74, Sex category

Clopidogrel for High Atherothrombotic

Risk and Ischemic Stabilization,

Management, and Avoidance

Confidence interval

Clopidogrel and the Optimization of

Gastrointestinal Events Trial

CABG Off or On Pump Revascularization

Study

Committee for Practice Guidelines

Creatinine clearance

Clopidogrel for the Reduction of Events

During Observation

Can Rapid risk stratification of Unstable angina patients Suppress ADverse outcomes with Early implementation of the ACC/AHA Guidelines

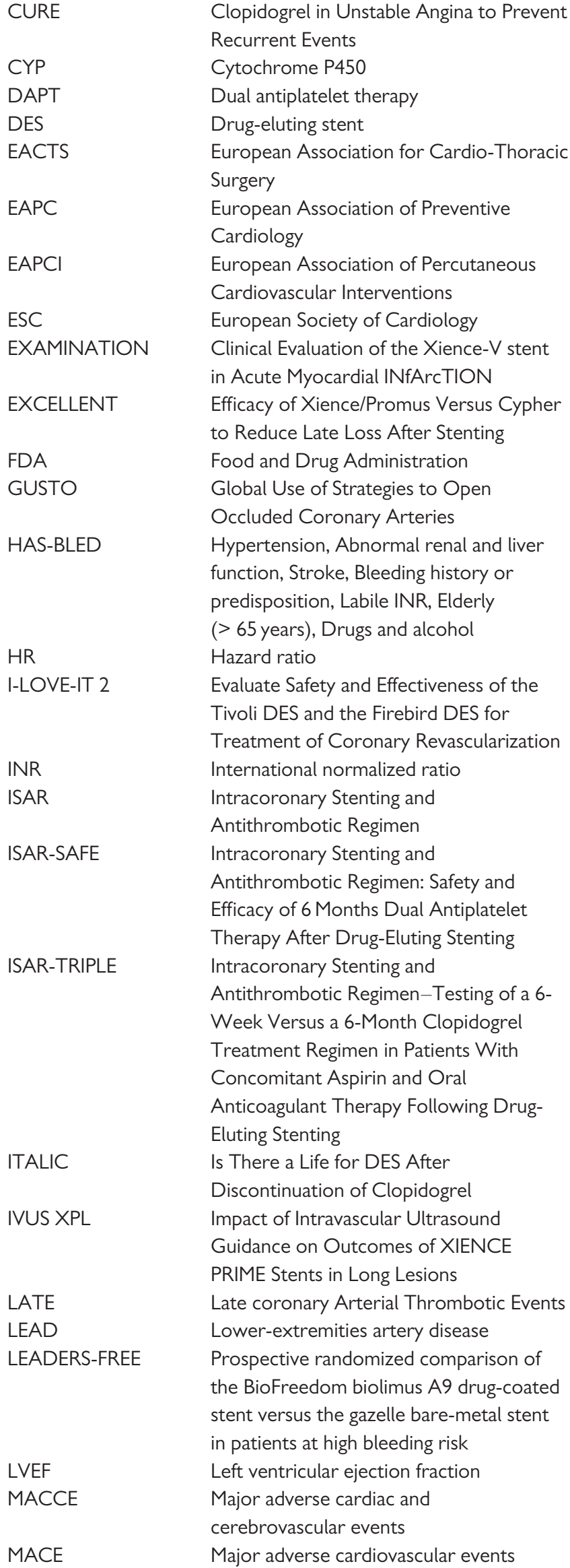


MATRIX

$\mathrm{MI}$

NACE

NCDR

NNT

NOAC

NORSTENT

NSTE-ACS

NSTEMI

OAC

o.d.

OPTIMIZE

OR

PARIS

$\mathrm{PCl}$

PEGASUS-TIMI 54

PIONEER AF-PCI

PLATO

PPI

PRECISE-DAPT

PRODIGY

PROTECT

q.d.

RCT

REDUAL-PCI

RESET

ROOBY

RR

RRR
Minimizing Adverse Haemorrhagic Events

by TRansradial Access Site and Systemic

Implementation of angioX

Myocardial infarction

Net adverse clinical events

National Cardiovascular Data Registry

Number needed to treat

Non-vitamin $\mathrm{K}$ oral anticoagulant

NORwegian coronary STENT trial

Non-ST elevation acute coronary syndrome

Non-ST-segment elevation myocardial

infarction

Oral anticoagulant

Omni die (once a day)

Optimized Duration of Clopidogrel

Therapy Following Treatment With the

Zotarolimus-Eluting Stent in Real-World

Clinical Practice

Odds ratio

Patterns of Nonadherence to Antiplatelet

Regimens in Stented Patients

Percutaneous coronary intervention

Prevention of Cardiovascular Events in

Patients with Prior Heart Attack Using

Ticagrelor Compared to Placebo on a

Background of Aspirin-Thrombolysis in

Myocardial Infarction 54

Rivaroxaban and a dose- adjusted oral

vitamin $\mathrm{K}$ antagonist treatment strategy in

subjects with atrial fibrillation who

undergo percutaneous coronary

intervention

PLATelet inhibition and patient Outcomes

Proton pump inhibitor

PREdicting bleeding Complications In

patients undergoing Stent implantation

and subsEquent Dual Anti Platelet

Therapy

PROlonging Dual antiplatelet treatment

after Grading stent-induced intimal

hYperplasia study

Patient-Related Outcomes With Endeavor

vs Cypher Stenting

Quaque die (one a day)

Randomized controlled trial

Evaluation of Dual Therapy With

Dabigatran vs. Triple Therapy With

Warfarin in Patients With AFib That

Undergo a PCl With Stenting

Real Safety and Efficacy of 3-Month Dual

Antiplatelet Therapy Following Endeavor

Zotarolimus-Eluting Stent Implantation

Veterans Affairs Randomized On/Off

Bypass trial

Relative risk

Relative risk reduction
SECURITY

Second Generation Drug-Eluting Stent Implantation Followed by Six- Versus

Twelve-Month Dual Antiplatelet Therapy

STEMI

STREAM

SYNTAX

ST-segment elevation myocardial

infarction

STrategic Reperfusion Early After

Myocardial Infarction

Synergy Between Percutaneous Coronary Intervention With Taxus and Cardiac

Surgery

TIA

TIMI

TL-PAS

TRA 2 ${ }^{\circ}$-TIMI 50

Transient ischaemic attack

Thrombolysis In Myocardial Infarction

Taxus Liberté Post Approval Study

Thrombin Receptor Antagonist in

Secondary Prevention of

Atherothrombotic Ischemic Events

TRACER

Thrombin Receptor Antagonist for

Clinical Event Reduction in Acute

Coronary Syndrome

TRILOGY ACS Targeted Platelet Inhibition to Clarify the

Optimal Strategy to Medically Manage

Acute Coronary Syndromes

TRITON-TIMI 38 Trial to Assess Improvement in Therapeutic Outcomes by Optimizing Platelet Inhibition with Prasugrel-Thrombolysis in Myocardial Infarction

TROPICAL-ACS Testing Responsiveness to Platelet Inhibition on Chronic Antiplatelet

Treatment For Acute Coronary

Syndromes Trial

VA

VKA

WOEST

Veterans' Administration

Vitamin $\mathrm{K}$ antagonist

What is the Optimal antiplatElet and anticoagulant therapy in patients with

$\mathrm{OAC}$ and coronary StenTing

ZES

ZEUS

Zotarolimus-eluting stent

Zotarolimus-eluting Endeavor sprint stent in Uncertain DES Candidates

$24 \mathrm{~h}$ a day, seven days a week

\section{Preamble}

Guidelines and Focused Updates written under the auspices of the European Society of Cardiology's (ESC) Committee for Practice Guidelines (CPG) summarize and evaluate available evidence with the aim of assisting health professionals in selecting the best management strategies for an individual patient with a given condition. The CPG Guidelines' and Focused Updates' recommendations should facilitate decision making of health professionals in their daily practice. However, the final decisions concerning an individual patient must be made by the responsible health professional(s) in consultation with the patient and caregiver as appropriate.

A great number of guidelines and focused updates have been issued in recent years by the ESC and by the European Association for Cardio-Thoracic Surgery (EACTS) as well as by other societies and organizations. Because of the impact on 
Table I Classes of recommendations

\begin{tabular}{|l|l|l}
\hline \multicolumn{1}{|c|}{$\begin{array}{c}\text { Classes of } \\
\text { recommendations }\end{array}$} & \multicolumn{1}{c}{ Definition } & $\begin{array}{c}\text { Suggested wording to } \\
\text { use }\end{array}$ \\
\hline Class I & $\begin{array}{l}\text { Evidence and/or general agreement } \\
\text { that a given treatment or } \\
\text { procedure is beneficial, useful, } \\
\text { effective. }\end{array}$ & $\begin{array}{l}\text { Is recommended/is } \\
\text { indicated }\end{array}$ \\
\hline Class II & $\begin{array}{l}\text { Conflicting evidence and/or a } \\
\text { divergence of opinion about the } \\
\text { usefulness/efficacy of the given } \\
\text { treatment or procedure. }\end{array}$ & \\
\hline Class IIa & $\begin{array}{l}\text { Weight of evidence/opinion is in } \\
\text { favour of usefulness/efficacy. }\end{array}$ & Should be considered \\
\hline Class IIb & $\begin{array}{l}\text { Usefulness/efficacy is less well } \\
\text { established by evidence/opinion. }\end{array}$ & May be considered \\
\hline Class III & $\begin{array}{l}\text { Evidence or general agreement that } \\
\text { the given treatment or procedure } \\
\text { is not useful/effective; and in some } \\
\text { cases may be harmful. }\end{array}$ & Is not recommended \\
\hline
\end{tabular}

clinical practice, quality criteria for the development of guidelines have been established in order to make all decisions transparent to the user. The recommendations for formulating and issuing ESC Guidelines can be found on the ESC website (http://www. escardio.org/Guidelines-\&-Education/Clinical-Practice-Guidelines/ Guidelines-development/Writing-ESC-Guidelines). ESC Guidelines represent the official position of the ESC on a given topic and are regularly updated.

Members of this Task Force were selected by the ESC and EACTS to represent professionals involved with the medical care of patients with this pathology. Selected experts in the field undertook a comprehensive review of the published evidence for management of a given condition according to ESC Committee for Practice Guidelines (CPG) policy and approved by the EACTS. A critical evaluation of diagnostic and therapeutic procedures was performed, including assessment of the risk-benefit ratio. The level of evidence and the strength of the recommendation of particular management options were weighed and graded according to predefined scales, as outlined in Tables 1 and 2.

The experts of the writing and reviewing panels provided declaration of interest forms for all relationships that might be perceived as real or potential sources of conflicts of interest. These forms were compiled into one file and can be found on the ESC website (http:// www.escardio.org/guidelines). Any changes in declarations of interest that arise during the writing period were notified to the ESC and updated. The Task Force received its entire financial support from the ESC without any involvement from the healthcare industry.

The ESC CPG supervises and coordinates the preparation of new Guidelines and of its Focused Updates. The Committee is also responsible for the endorsement process of these documents. These CPG documents undergo extensive review by the CPG and external
Table 2 Levels of evidence

\begin{tabular}{l|l}
\hline $\begin{array}{l}\text { Level of } \\
\text { evidence A }\end{array}$ & $\begin{array}{l}\text { Data derived from multiple randomized } \\
\text { clinical trials or meta-analyses. }\end{array}$ \\
\hline $\begin{array}{l}\text { Level of } \\
\text { evidence B }\end{array}$ & $\begin{array}{l}\text { Data derived from a single randomized } \\
\text { clinical trial or large non-randomized } \\
\text { studies. }\end{array}$ \\
\hline $\begin{array}{l}\text { Level of } \\
\text { evidence C }\end{array}$ & $\begin{array}{l}\text { Consensus of opinion of the experts and/ } \\
\text { or small studies, retrospective studies, } \\
\text { registries. }\end{array}$ \\
\hline
\end{tabular}

experts, and in this case by EACTS-appointed experts. After appropriate revisions the CPG documents are approved by all the experts involved in the Task Force. The finalized document is approved by the CPG for publication in the European Heart Journal and in the European Journal of Cardio-Thoracic Surgery. These CPG documents were developed after careful consideration of the scientific and medical knowledge and the evidence available at the time of their dating.

The task of developing this CPG Focused Update in collaboration with EACTS also includes the creation of educational tools and implementation programmes for the recommendations including condensed pocket guideline versions, summary slides, and an electronic version for digital applications (smartphones, etc.) as well as other educational tools depending on the topic. These versions are abridged and thus, if needed, one should always refer to the full text version, which is freely available via the ESC website and hosted on the EHJ website. The National Societies of the ESC are encouraged 
to endorse, translate and implement all ESC Guidelines. Implementation programmes are needed because it has been shown that the outcome of disease may be favourably influenced by the thorough application of clinical recommendations.

Surveys and registries are needed to verify that real-life daily practice is in keeping with what is recommended in the guidelines, thus completing the loop between clinical research, writing of guidelines and official focused updates, disseminating them and implementing them into clinical practice.

Health professionals are encouraged to take the ESC CPG Guidelines and Focused Updates developed in collaboration with EACTS fully into account when exercising their clinical judgment, as well as in the determination and the implementation of preventive, diagnostic or therapeutic medical strategies. However, the CPG documents do not override in any way whatsoever the individual responsibility of health professionals to make appropriate and accurate decisions in consideration of each patient's health condition and in consultation with that patient or the patient's caregiver where appropriate and/or necessary. It is also the health professional's responsibility to verify the rules and regulations applicable to drugs and devices at the time of prescription.

\section{Introduction}

The estimated number of patients requiring dual antiplatelet therapy (DAPT), consisting of the combination of aspirin and an oral inhibitor of the platelet $\mathrm{P} 2 \mathrm{Y}_{12}$ receptor for adenosine 5'-diphosphate (ADP), is considerable and has increased over time in Europe. Based on population estimates from 2015, in the region of 1400000 and 2200000 patients per year may have an indication for DAPT after coronary intervention or myocardial infarction (MI), respectively. ${ }^{1}$

This year, 2017, is the 21st anniversary of the publication of the first randomized clinical trial to establish the superiority of DAPT over anticoagulant therapy among patients undergoing percutaneous coronary intervention (PCl) (Figure 1). ${ }^{2}$ Based on over 35 randomized clinical trials, including more than 225000 patients, DAPT is among the most intensively investigated treatment options in the field of cardiovascular medicine. Along with progressive refinement of $\mathrm{P}_{2} \mathrm{Y}_{12}$ inhibition strategies - embracing firstly safer (from ticlopidine to clopidogrel) and then more potent and predictable (from clopidogrel to ticagrelor or prasugrel) drugs — research has concomitantly focused on optimal treatment duration. The need to investigate longer DAPT regimens firstly arose from concerns over late and very

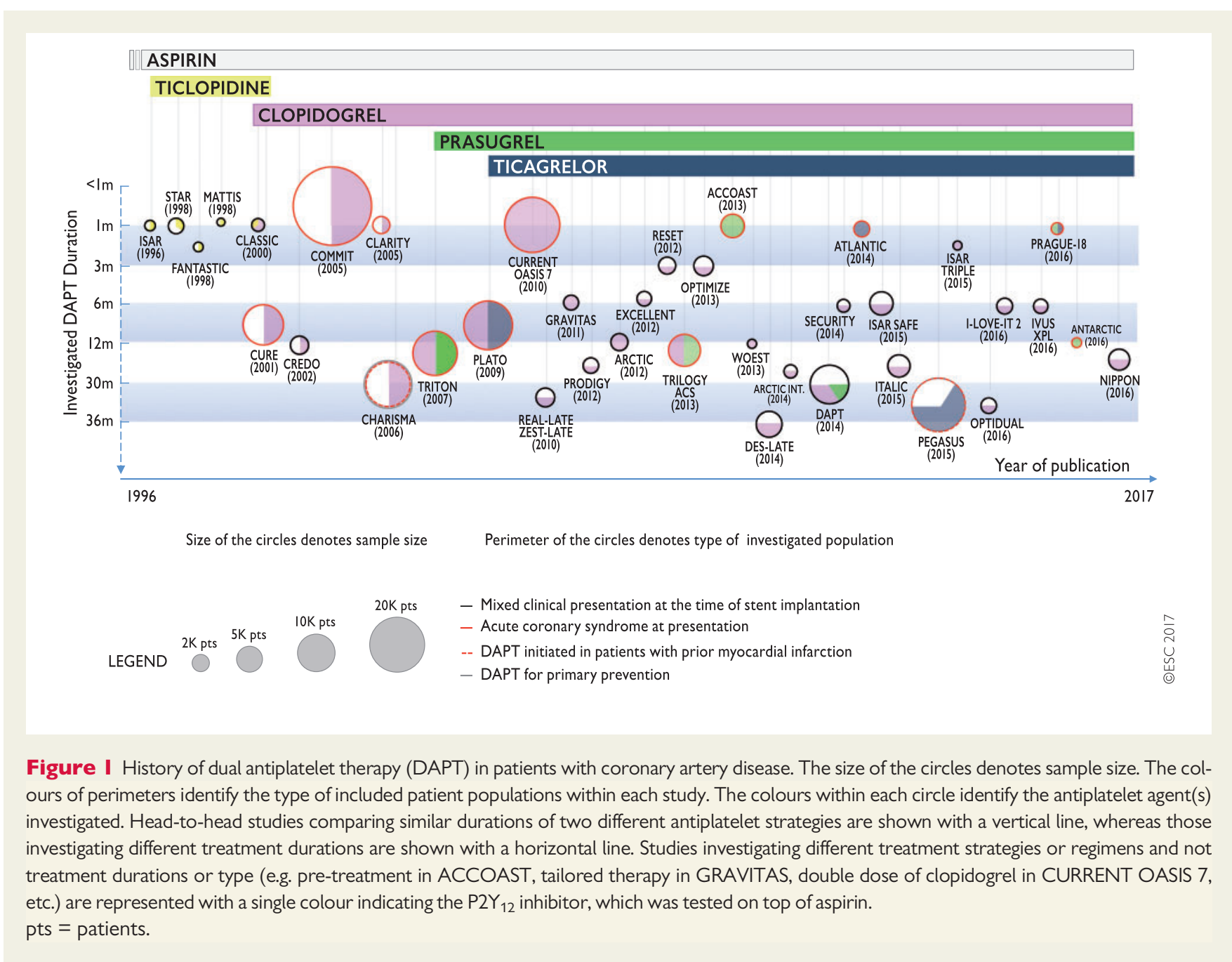


late stent thrombosis occurring after first-generation drug-eluting stent (DES) implantation. ${ }^{3}$ Yet, the advent of safer newer-generation DESs and the results of the most recent randomized controlled trials (RCTs) have established a major paradigm shift in the way DAPT should be conceived and used in clinical practice. DAPT remains a highly effective preventive treatment for stent thrombosis across the board; however, the risks of late and (even more) very late stent thrombosis have declined considerably since the advent of newergeneration DESs. Hence, the risk of bleeding associated with DAPT prolongation beyond 1 year does not seem to be justified by the small absolute benefit observed in terms of very late stent thrombosis prevention. On the other hand, there is emerging evidence that DAPT reduces the long-term risk of non-stent-related $\mathrm{MI}$ as well as stroke. Hence, after 21 years of research, DAPT has moved from a local (i.e. stent-related) to a systemic treatment strategy (i.e. capable of preventing thrombotic arterial vessel occlusion), conveying global patient protection (Figure 1).

There is, however, confusion in the community around the optimal type and duration of DAPT in patients with established coronary artery disease (CAD), undergoing coronary revascularization or not. ${ }^{4}$ This derives from apparently conflicting results arising from the available studies and limited evidence on various patient subsets (e.g. elderly patients, with comorbidities or at greater bleeding risk) in whom the trade-off between the benefits and risks of DAPT may differ from those observed in more selected patient cohorts included in trials. Therefore, the scope of this focused update is to address recommendations on DAPT in patients with CAD.

\subsection{Short- and long-term outcomes after percutaneous coronary intervention}

See Web Addenda.

\subsection{Risk of stent thrombosis in relation to stent type}

See Web Addenda.

\subsection{Short- and long-term outcomes after coronary artery bypass surgery}

See Web Addenda.

\subsection{Short- and long-term outcomes after medically managed acute coronary syndrome}

See Web Addenda.

\section{Efficacy and safety of dual antiplatelet therapy and risk stratification tools}

Current evidence suggests that DAPT mitigates the risk of stent thrombosis across the whole spectrum, from acute to very late events. However, treatment with DAPT beyond 1 year after MI, or after $\mathrm{PCl}$, exerts the majority of its benefit by reducing the rate of spontaneous $\mathrm{MI}$, which is associated with mortality rates of $15 \%{ }^{5}$
Nonetheless, because continued antiplatelet therapy is also associated with increased bleeding risk, it is necessary to weigh this risk against the potential benefit. Current evidence suggests that the risk of bleeding in patients on DAPT is proportionally related to its duration both within and beyond 1 year of treatment duration. Since the benefits of prolonged DAPT, especially for mortality endpoints, appear highly dependent on prior cardiovascular history [such as prior acute coronary syndrome (ACS)/MI vs. stable CAD], and prediction models to estimate on-DAPT bleeding risk have been developed, an individualized approach based on ischaemic vs. bleeding risk assessment is warranted

\subsection{Dual antiplatelet therapy for the prevention of stent thrombosis \\ See Web Addenda.}

\subsection{Dual antiplatelet therapy for the prevention of spontaneous myocardial infarction}

See Web Addenda.

\subsection{Dual antiplatelet therapy and mortality rate}

See Web Addenda.

\subsection{Safety of dual antiplatelet therapy} See Web Addenda.

\subsection{Risk stratification tools for ischaemia and bleeding risks}

Given the trade-off between ischaemic vs. bleeding risks for any given DAPT duration, the use of scores might prove useful to tailor DAPT duration in order to maximize ischaemic protection and minimize bleeding risks in the individual patient. ${ }^{6}$ Most of the frequently used risk scores for assessing ischaemic events ${ }^{7-9}$ and major bleeds ${ }^{10-12}$ were originally developed and validated for the prediction of events occurring mainly during hospital stay or early on thereafter. ${ }^{13,14}$ As a result, the application of these risk scores to decide upon DAPT duration remains problematic, as only limited data exist exploring their value to guide DAPT duration. ${ }^{13}$ On the other hand, the use of risk scores that were specifically designed to guide and inform decision making on DAPT duration should be prioritized over other available risk scores (Table 3).

The DAPT score was developed from 11648 patients enrolled in the DAPT trial and was initially validated in 8136 patients enrolled in the Patient-Related Outcomes With Endeavor vs. Cypher Stenting (PROTECT) trial. $^{15}$ This prediction rule identified nine factors [age, congestive heart failure/low left ventricular ejection fraction (LVEF), vein graft stenting, $\mathrm{Ml}$ at presentation, prior $\mathrm{Ml}$ or $\mathrm{PCl}$, diabetes, stent diameter $<3 \mathrm{~mm}$, smoking, and paclitaxel-eluting stent] resulting in a score ranging from -2 to +10 . Within the DAPT trial, a high-risk score (i.e. a score $\geq 2$ ) selected patients who showed a reduction in $\mathrm{Ml} / \mathrm{stent}$ thrombosis and cardiovascular or cerebrovascular events risk [number needed to treat (NNT) for benefit for ischaemic event reduction $=34$ ] after a prolonged, 30-month DAPT, with only a modest increase in bleeding risk (NNT for harm $=272$ ). In turn, a 
Table 3 Risk scores validated for dual antiplatelet therapy duration decision-making

\begin{tabular}{|c|c|c|c|c|}
\hline & PRECISE-DAPT score ${ }^{18}$ & \multicolumn{2}{|c|}{ DAPT score ${ }^{15}$} & \\
\hline Time of use & At the time of coronary stenting & \multicolumn{3}{|c|}{ After 12 months of uneventful DAPT } \\
\hline $\begin{array}{l}\text { DAPT duration } \\
\text { strategies assessed }\end{array}$ & $\begin{array}{c}\text { Short DAPT ( } 3-6 \text { months) } \\
\text { vs. } \\
\text { Standard/long DAPT (12-24 months) }\end{array}$ & \multicolumn{2}{|c|}{$\begin{array}{c}\text { Standard DAPT (12 months) } \\
\text { vs. } \\
\text { Long DAPT ( } 30 \text { months) }\end{array}$} & \\
\hline Score calculation ${ }^{\mathrm{a}}$ & 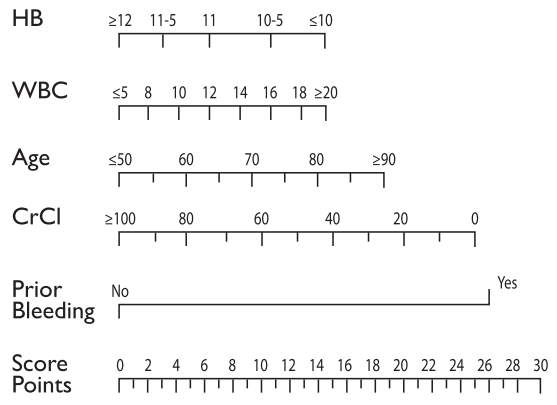 & $\begin{array}{l}\text { Age } \\
\quad \geq 75 \\
\quad 65 \text { to }<75 \\
\quad<65 \\
\text { Cigarette smoking } \\
\text { Diabetes mellitus } \\
\text { MI at presentation } \\
\text { Prior PCl or prior MI } \\
\text { Paclitaxel-eluting stent } \\
\text { Stent diameter }<3 \mathrm{~mm} \\
\text { CHF or LVEF }<30 \% \\
\text { Vein graft stent }\end{array}$ & $\begin{array}{r}-2 \mathrm{pt} \\
-1 \mathrm{pt} \\
0 \mathrm{pt} \\
+1 \mathrm{pt} \\
+1 \mathrm{pt} \\
+1 \mathrm{pt} \\
+1 \mathrm{pt} \\
+1 \mathrm{pt} \\
+1 \mathrm{pt} \\
+2 \mathrm{pt} \\
+2 \mathrm{pt}\end{array}$ & \\
\hline Score range & 0 to 100 points & \multicolumn{2}{|c|}{-2 to 10 points } & \\
\hline $\begin{array}{l}\text { Decision making cut-off } \\
\text { suggested }\end{array}$ & $\begin{array}{c}\text { Score } \geq 25 \rightarrow \text { Short DAPT } \\
\text { Score }<25 \rightarrow \text { Standard/long DAPT }\end{array}$ & \multicolumn{2}{|c|}{$\begin{array}{c}\text { Score } \geq 2 \rightarrow \text { Long DAPT } \\
\text { Score }<2 \rightarrow \text { Standard DAPT }\end{array}$} & 슴 \\
\hline Calculator & www.precisedaptscore.com & \multicolumn{2}{|c|}{ www.daptstudy.org } & 峞 \\
\hline $\begin{array}{l}=\text { congestive heart failur } \\
\text { ction; PCI = percutaneou } \\
\text { Platelet Therapy; WBC = } \\
\text { the PRECISE-DAPT scor } \\
\text { rmine the number of poin } \\
\text { e calculation is provided ir } \\
\text { the DAPT score summate }\end{array}$ & $\begin{array}{l}\mathrm{Cl}=\text { creatinine clearance; DAPT = dual antiplatelet the } \\
\text { ronary intervention; PRECISE-DAPT = PREdicting blee } \\
\text { te blood cell count. } \\
\text { the score nomogram: mark patient's value for each of } \\
\text { btained for each clinical variable. Then summate the poir } \\
\text { b Figure } 1 \text { of the Web Addenda. } \\
\text { tive points for each value and subtract values for age to }\end{array}$ & $\begin{array}{l}\mathrm{Hb}=\text { haemoglobin; LVEF } \\
\text { Lomplications In patients } \\
\text { ve clinical variables of the } \\
\text { tained for each clinical var } \\
\text { tal score. }\end{array}$ & $\begin{array}{l}\text { on fracti } \\
\text { tation a } \\
\text { cal line } \\
\text { A practi }\end{array}$ & $\begin{array}{l}\text { cardial } \\
\text { it Dual } \\
\text { axis to } \\
\text { ple for }\end{array}$ \\
\hline
\end{tabular}

low-risk score $(<2)$ selected patients recruited in the DAPT trial who did not derive any reduction of ischaemic events from prolonging DAPT, with a significant increase in moderate/major bleeding (NNT for harm =64). As DAPT duration was not randomized in the PROTECT trial, the value of the DAPT score in guiding the duration of therapy has so far only been shown for patients recruited to the DAPT trial. Additional validation of the DAPT score to guide DAPT duration is needed, especially in the context of less well-selected patients as compared to those recruited in the DAPT trial and undergoing treatment with new-generation DES only.

Two independent predictive scores for bleeding [age, body mass index, smoking, anaemia, creatinine clearance $(\mathrm{CrCl})$, and triple therapy at discharge] and $\mathrm{MI}$ or stent thrombosis [diabetes mellitus, ACS, smoking, $\mathrm{CrCl}$, prior $\mathrm{PCl}$, and prior coronary artery bypass graft surgery $(\mathrm{CABG})$ ] have also been developed from the Patterns of Nonadherence to Antiplatelet Regimens in Stented Patients (PARIS) registry. ${ }^{16}$ PARIS was a prospective, multicentre, observational study of patients undergoing $\mathrm{PCl}$ with stent implantation in the USA and Europe, which was designed to examine the different modes of DAPT cessation and to investigate the influence of these modes on subsequent clinical adverse events. ${ }^{17}$ This registry study included patients with an indication for oral anticoagulation. The value of the PARIS bleeding and/or ischaemic risk scores to tailor DAPT duration remains unclear, since therapy duration was not randomized in the PARIS study and no study to date has applied the results of these scores for DAPT type or duration guidance. A high ischaemic risk status was observed in roughly $40 \%$ of high bleeding risk patients ${ }^{16}$ and as many as $65.3 \%$ presented low ischaemic and bleeding risks. ${ }^{16}$ Therefore, it remains unclear how DAPT duration should be guided by the simultaneous assessment of ischaemic and bleeding risk features according to PARIS.

The PRECISE-DAPT (PREdicting bleeding Complications In patients undergoing Stent implantation and subsEquent Dual Anti Platelet Therapy) collaborative study included a total of 14963 patients with CAD who underwent elective, urgent, or emergent $\mathrm{PCl}$ and generated a five-item (age, $\mathrm{CrCl}$, haemoglobin, white blood cell count, and prior spontaneous bleeding) prediction algorithm for outof-hospital bleeding in patients treated with DAPT. ${ }^{18}$

The predictive performance of this novel score was assessed in the derivation cohort and validated in 8595 and 6172 patients treated with PCl from the PLATelet inhibition and patient Outcomes (PLATO) trial and the Bern PCl registry, ${ }^{19,20}$ respectively. The PRECISE-DAPT score showed improved integrated discrimination and reclassification performance as compared to the PARIS bleeding score in both validation cohorts. $^{18}$ The usefulness of this score was also assessed within patients randomized to different DAPT durations $(n=10081)$ to identify the effect on bleeding and ischaemia of a long (12-24 months) or short (3-6 months) treatment duration in relation to baseline bleeding risk. It was observed that among patients deemed at high bleeding risk based on PRECISE-DAPT (PRECISE-DAPT score $\geq 25$ ), prolonged 
DAPT was associated with no ischaemic benefit but a remarkable bleeding burden leading to an NNT for harm of $38 .{ }^{18}$ On the other hand, longer treatment in patients without high bleeding risk (PRECISE-DAPT score $<25$ ) was associated with no increase in bleeding and a significant reduction in the composite ischaemic endpoint of $\mathrm{Ml}$, definite stent thrombosis, stroke, and target vessel revascularization, with an NNT for benefit of $65 .^{18}$ Selecting a shorter than 12month treatment duration in patients deemed at high bleeding risk upfront may therefore prevent their exposure to an excessive bleeding hazard. In turn, patients at non-high bleeding risk might receive a standard (i.e. 12 months) or prolonged (i.e. $>12$ months) course of treatment if tolerated.

However, none of these risk prediction models have been prospectively tested in the setting of RCTs. Therefore, their value in improving patient outcomes remains unclear.

\section{Use of risk scores as guidance for the duration of dual antiplatelet therapy}

\begin{tabular}{|l|c|c|}
\hline Recommendations & Class $^{\mathrm{a}}$ & Level $^{\mathrm{b}}$ \\
\hline $\begin{array}{l}\text { The use of risk scores designed to evaluate } \\
\text { the benefits and risks of different DAPT } \\
\text { durations }^{\mathrm{c}} \text { may be considered. }\end{array}$ & Il, 18 & $\mathbf{A}$ \\
\hline
\end{tabular}

DAPT $=$ dual antiplatelet therapy.

${ }^{\mathrm{a} C}$ Class of recommendation.

'Level of evidence.

'The DAPT and PRECISE-DAPT scores are those currently fulfilling these requirements.

\subsection{Type of $\mathrm{P}_{2} \mathrm{Y}_{12}$ inhibitor and timing of initiation}

Clopidogrel: Clopidogrel is associated with a better safety profile than ticlopidine, mainly in terms of allergy, skin or gastrointestinal disorders, and neutropenia, while it has a similar degree and consistency of $\mathrm{P}_{2} \mathrm{Y}_{12}$ inhibition and bleeding risk. ${ }^{21,22}$ The wide variability in the pharmacodynamic response to ticlopidine and clopidogrel is linked to several factors, including genotype polymorphisms. ${ }^{22}$ Clinical evidence with respect to the optimal duration of clopidogrel therapy after $\mathrm{PCl}$ is discussed elsewhere (Chapter 4).

Prasugrel: Prasugrel achieves a faster, greater, and more consistent degree of $\mathrm{P}_{2} \mathrm{Y}_{12}$ inhibition as compared to clopidogrel. Prasugrel requires two metabolic steps for formation of its active metabolite, which is chemically similar to the active metabolite of clopidogrel. The Trial to Assess Improvement in Therapeutic Outcomes by Optimizing Platelet Inhibition with Prasugrel-Thrombolysis in Myocardial Infarction (TRITON-TIMI 38) included P2Y 12 inhibitornaïve ACS patients in whom coronary anatomy was deemed suitable for $\mathrm{PCl}$, or patients with ST-segment elevation myocardial infarction (STEMI) referred for primary $\mathrm{PCl}^{23}$ Duration of DAPT was up to 15 months in both study arms. The composite primary endpoint (cardiovascular death, non-fatal MI, or stroke) occurred in $9.3 \%$ of prasugrel-treated patients vs. $11.2 \%$ of clopidogrel-treated patients [hazard ratio (HR) 0.82, 95\% confidence interval (Cl) 0.73-0.93; $P=$ 0.002], mostly driven by a significant risk reduction for MI [from $9.2 \%$ to $7.1 \%$; relative risk reduction (RRR) $23.9 \%, 95 \% \mathrm{Cl} 12.7-33.7$; $P<$ 0.001). ${ }^{23}$ There was no difference in the rates of either non-fatal stroke or cardiovascular death.

Prasugrel was associated with a significant increase in the rate of non-CABG-related TIMI major bleeding (2.4\% vs. 1.8\%; HR 1.32, 95\% Cl 1.03-1.68; $P=0.03$ ). Life-threatening bleeding was significantly increased under prasugrel compared with clopidogrel (1.4\% vs. $0.9 \%$; HR 1.52, 95\% Cl 1.08-2.13; $P=0.01)$, as was fatal bleeding (0.4\% vs. 0.1\%, HR 4.19, 95\% Cl 1.58-11.11; $P=0.002)$. CABG-related bleeding was also higher in prasugrel-treated patients (13.4\% vs. $3.2 \%$; HR 4.72, 95\% Cl 1.90-11.82; $P<0.001)$. There was evidence of net harm with prasugrel in patients with a history of cerebrovascular events. In addition, there was no apparent net clinical benefit in patients $\geq 75$ years of age and in patients with low body weight $(<60 \mathrm{~kg}){ }^{23}$ Prasugrel was not tested in medically managed ACS patients in the setting of the TRITON-TIMI 38 study. In the Targeted Platelet Inhibition to Clarify the Optimal Strategy to Medically Manage Acute Coronary Syndromes (TRILOGY ACS) study, which exclusively included medically managed ACS patients, the primary endpoint of death from cardiovascular causes, MI, or stroke among patients under the age of 75 years occurred in $13.9 \%$ of the prasugrel group and $16.0 \%$ of the clopidogrel group (HR 0.91, 95\% $\mathrm{Cl} 0.79-1.05 ; P=$ $0.21)$, at a median follow-up of 17 months. ${ }^{24}$ Similar results were observed in the overall population (i.e. also including elderly patients). Hence, prasugrel is not indicated in medically managed ACS patients.

The TRITON-TIMI 38 study mandated the use of prasugrel or clopidogrel after coronary angiography if an indication to proceed to $\mathrm{PCl}$ was established. Pre-treatment was allowed only in STEMI patients undergoing primary intervention $(n=2438)$.

For the comparison of prasugrel at the time of $\mathrm{PCl}$, in the $\mathrm{A}$ Comparison of Prasugrel at the Time of Percutaneous Coronary Intervention Or as Pretreatment At the Time of Diagnosis in Patients with Non-ST-Elevation Myocardial Infarction (ACCOAST), 4033 patients with non-STEMI (NSTEMI) who were scheduled to undergo coronary angiography within 2-48 h after randomization were assigned to receive prasugrel (a $30 \mathrm{mg}$ loading dose) before angiography (pretreatment group) or placebo (control group). ${ }^{25}$ When $\mathrm{PCl}$ was indicated, an additional $30 \mathrm{mg}$ of prasugrel was given in the pre-treatment group at the time of $\mathrm{PCl}$ and $60 \mathrm{mg}$ of prasugrel was given in the control group. The rate of the primary efficacy endpoint, a composite of death from cardiovascular causes, MI, stroke, urgent revascularization, or glycoprotein Ilb/llla inhibitor rescue therapy (glycoprotein Ilb/llla bailout) through day 7 , did not differ significantly between the two groups (HR with pre-treatment $1.02,95 \% \mathrm{Cl} 0.84-1.25 ; P=0.81) .{ }^{25}$ The rate of the key safety endpoint of all TIMI major bleeding episodes, whether related to CABG or not, through day 7 was increased with pre-treatment $(H R$ 1.90, 95\% Cl 1.19-3.02; $P=0.006)$. The rates of TIMI major bleeding and life-threatening bleeding not related to CABG were increased by a factor of 3 and 6, respectively. Pre-treatment did not reduce the rate of the primary outcome among patients undergoing $\mathrm{PCl}(69 \%$ of the patients) but increased the rate of TIMI major bleeding at 7 days. ${ }^{25}$

Hence, prasugrel is not indicated in patients with ACS in whom coronary anatomy is not known and an indication for $\mathrm{PCl}$ is not clearly established, with the exception of STEMI patients scheduled to undergo immediate coronary catheterization and $\mathrm{PCl}$, if clinically indicated.

In the DAPT trial, 3461 patients (34.7\% of the total trial population) who were treated with prasugrel within the first 12 months after 
intervention were randomly allocated to stop or continue the treatment for an additional 18 months. ${ }^{26}$ The type of $\mathrm{P}_{2} \mathrm{Y}_{12}$ inhibitor or stent type were not randomized for. However, the largest cohort of prasugrel-treated patients $(n=2191)$ was provided by the TAXUS Liberté Post Approval Study (TL-PAS), which was a prospective, multicentre, open-label study developed to review the clinical performance of the Taxus Liberté paclitaxel-eluting stent in routine clinical practice in the USA. $^{27}$ Enrolled TL-PAS patients received open-label prasugrel plus aspirin for 12 months after stent placement; enrolment was not restricted to patients presenting with ACS (i.e. those with an approved indication for prasugrel). Rates of death and stroke were similar between groups, but $\mathrm{Ml}$ was significantly reduced with prolonged prasugrel treatment (1.9\% vs. 7.1\%; HR 0.255; $P<0.001)$. The DAPT coprimary endpoint, stent thrombosis, was also lower with longer therapy (0.2\% vs. 2.9\%; HR 0.063; $P<0.001$ ). The safety endpoint of GUSTO (Global Use of Strategies to Open Occluded Coronary Arteries) moderate or severe bleeding was numerically increased in the patients continuing prasugrel to 30 months, although the difference was not statistically significant ( $2.4 \%$ vs. $1.7 \%$; HR $1.438 ; P=0.234) .{ }^{27}$ No subgroup data have been provided among patients treated with prasugrel with respect to indication for $\mathrm{PCl}$ (i.e. ACS vs. stable CAD) or type of implanted stent (i.e. paclitaxel-eluting stent vs. other stent types).

Ticagrelor: Ticagrelor belongs to a novel chemical class, cyclopentyl triazolopyrimidine, and is a direct oral, reversibly binding P2 $\mathrm{Y}_{12}$ inhibitor with a plasma half-life of $\sim 12 \mathrm{~h}$. In the PLATO trial, ticagrelor proved to be superior to clopidogrel in ACS patients, who were allowed to be pre-treated with clopidogrel at hospital admission, irrespective of the final revascularization strategy (i.e. planned or not planned invasive management). ${ }^{20}$ Patients with either moderate- to high-risk non-ST elevation ACS (NSTE-ACS) (planned for either conservative or invasive management) or STEMI planned for primary $\mathrm{PCl}$ were randomized to either clopidogrel $75 \mathrm{mg}$ daily, with a loading dose of $300 \mathrm{mg}$, or ticagrelor $180 \mathrm{mg}$ loading dose followed by $90 \mathrm{mg}$ twice daily. ${ }^{20}$ Patients undergoing $\mathrm{PCl}$ were allowed to receive an additional blinded $300 \mathrm{mg}$ loading dose of clopidogrel (total loading dose $600 \mathrm{mg}$ ) or its placebo, and were also recommended to receive an additional $90 \mathrm{mg}$ of ticagrelor (or its placebo) if $>24 \mathrm{~h}$ after the initial loading dose. Treatment was continued for up to 12 months, with a minimum intended treatment duration of 6 months and a median duration of study drug exposure of 9 months. ${ }^{20}$

In the overall cohort, the primary composite efficacy endpoint (death from vascular causes, Ml, or stroke) was observed in $9.8 \%$ of the patients in the ticagrelor group and in $11.7 \%$ of the patients in the clopidogrel group (HR 0.84, 95\% Cl 0.77-0.92; $P<0.001) .{ }^{20}$ According to the pre-defined statistical analysis plan, death from vascular causes was significantly reduced from $5.1 \%$ to $4.0 \%$ (HR 0.79 , 95\% Cl 0.69-0.91; $P=0.001)$, and from $\mathrm{Ml}$ from $6.9 \%$ to $5.8 \%(\mathrm{HR}$ $0.84,95 \% \mathrm{Cl} 0.75-0.95 ; P=0.005)$. There was no significant difference in the rates of stroke (1.3\% vs. $1.5 \%$; $P=0.22)$. The rate of definite stent thrombosis was reduced from $1.9 \%$ to $1.3 \%(P<0.01)$ and total mortality from $5.9 \%$ to $4.5 \%(P<0.001)$. Overall, there was no significant difference in PLATO-defined major bleeding rates between the clopidogrel and ticagrelor groups ( $11.2 \%$ vs. $11.6 \%$, respectively; $P=$ 0.43). Major bleeding unrelated to CABG was increased from $3.8 \%$ in the clopidogrel group to $4.5 \%$ in the ticagrelor group (HR 1.19, $95 \%$ Cl 1.02-1.38; $P=0.03)$. Major bleeding related to CABG was similar with ticagrelor and clopidogrel (7.4\% vs. $7.9 \%$, respectively; $P=0.32)$.
There was no difference in the overall rates of fatal haemorrhage between the groups ( $0.3 \%$ in both groups). The superiority of ticagrelor over clopidogrel with respect to the primary study endpoint as well as cardiovascular death or overall mortality was consistent across management strategies, i.e. patients undergoing $\mathrm{PCl}$, those medically managed, and patients who underwent CABG. ${ }^{20}$

No dedicated study exists assessing the value of early (i.e. before coronary angiography) vs. delayed (i.e. after coronary angiography) ticagrelor administration in patients with NSTE-ACS. The Administration of Ticagrelor in the Cath Lab or in the Ambulance for New ST Elevation Myocardial Infarction to Open the Coronary Artery (ATLANTIC) study involved 1862 patients with STEMI <6-h duration and compared pre-hospital (in the ambulance) vs. in-hospital (in the catheterization laboratory) treatment with ticagrelor. ${ }^{28}$ The coprimary endpoints were the proportion of patients who did not have $\geq 70 \%$ resolution of ST-segment elevation before $\mathrm{PCl}$ and the proportion of patients who did not have TIMI flow grade 3 in the infarctrelated artery at initial angiography. Secondary endpoints included the rates of major adverse cardiovascular events (MACE) and definite stent thrombosis at 30 days. The median time difference between the two treatment strategies was $31 \mathrm{~min}$. The two co-primary endpoints did not differ significantly between the pre-hospital and in-hospital groups. The rates of definite stent thrombosis were lower in the prehospital group than in the in-hospital group ( $0 \%$ vs. $0.8 \%, P=0.008$ in the first $24 \mathrm{~h} ; 0.2 \%$ vs. $1.2 \%, P=0.02$ at 30 days). Rates of major bleeding events were low and virtually identical in the two groups, regardless of the bleeding definition used. ${ }^{28}$

The value of ticagrelor beyond 12 months of therapy in patients with prior ACS has been investigated in the Prevention of Cardiovascular Events in Patients with Prior Heart Attack Using Ticagrelor Compared to Placebo on a Background of Aspirin-Thrombolysis in Myocardial Infarction (PEGASUS) trial, which is described in Chapter $4 .^{29}$

$P 2 Y_{12}$ inhibitors in STEMI patients treated with lysis: Clopidogrel is the only $\mathrm{P}_{2} \mathrm{Y}_{12}$ inhibitor that has been properly investigated in patients with STEMI undergoing initial treatment with thrombolysis. ${ }^{31,32}$ Clopidogrel $300 \mathrm{mg}$ loading dose has been investigated only in patients $\leq 75$ years of age. ${ }^{31}$ While not specifically investigating the value of clopidogrel, in the STrategic Reperfusion Early After Myocardial Infarction (STREAM) study, patients aged 75 or more received clopidogrel treatment without loading dose (i.e. initiated at $75 \mathrm{mg}$ q.d.) in association with half dose of lytic therapy. ${ }^{30}$ Hence, the administration of a clopidogrel loading dose in elderly patients requires a patient-by-patient decision. While prasugre ${ }^{33}$ or ticagre$\operatorname{lor}^{20}$ were allowed as per protocol in patients with prior treatment with lysis in $\mathrm{P}_{2} \mathrm{Y}_{12}$ inhibitor-naive patients or those with prior clopidogrel administration, respectively, there are insufficient safety data to recommend their concomitant use during or soon after thrombolysis.

Timing of initiation of $\mathrm{P}_{2} \mathrm{Y}_{12}$ inhibitor: The evidence (and lack thereof) on optimal timing for the initiation of $\mathrm{P}_{2} \mathrm{Y}_{12}$ inhibitors has been extensively discussed in previous guidelines ${ }^{34}$ and reviewed elsewhere. ${ }^{35,36}$ A reasonable approach is to start treatment with a $P 2 Y_{12}$ inhibitor based on the timing with which the drug was investigated in approval studies (i.e. start as soon as possible and deemed safe for clopidogrel and ticagrelor or after the indication for $\mathrm{PCl}$ is established based on coronary anatomy for prasugrel). The decision to withhold early administration of $\mathrm{P}_{2} \mathrm{Y}_{12}$ inhibitors may also depend on planned 
use of cangrelor in the catheterization laboratory, which ensures immediate inhibition of the target receptor in oral $\mathrm{P}_{2} \mathrm{Y}_{12}$ inhibitornaïve patients. Timing of administration of $\mathrm{P}_{2} \mathrm{Y}_{12}$ inhibitors in patients receiving cangrelor infusion at the time of $\mathrm{PCl}$ should be drug-specific. ${ }^{37}$ While ticagrelor can be given any time before, during or at the end of cangrelor infusion, it is recommended that clopidogrel or prasugrel is given the time of cangrelor infusion discontinuation (or within 30 minutes before the end of infusion in the case of prasugrel administration). ${ }^{37}$ However, the comparative efficacy and safety of routine early oral $\mathrm{P}_{2} \mathrm{Y}_{12}$ inhibitor administration vs. the use of cangrelor in the catheterization laboratory in patients with ACS undergoing invasive management deserves further investigation. If coronary anatomy is known or the probability of $\mathrm{PCl}$ is high (such as for STEMI patients), there is evidence and general consensus that early administration of oral $\mathrm{P}_{2} \mathrm{Y}_{12}$ inhibitors outweighs any potential risks. On the other hand, there are no convincing data that the benefits of early administration of a P2Y 12 inhibitor outweigh the possible risks in stable CAD patients undergoing diagnostic angiography.

\subsection{Measures to minimize bleeding while on dual antiplatelet therapy}

Bleeding events after successful $\mathrm{PCl}$ are independently associated with increased mortality and morbidity and this association is likely causal. ${ }^{41,42}$ Therefore, every effort should be made to minimize bleeding. Individualization of therapy is a key measure and includes the identification of risk factors for bleeding, radial access site, dosing of therapies, use of proton pump inhibitors (PPIs), and appropriate selection of $\mathrm{P}_{2} \mathrm{Y}_{12}$ inhibitors.

Vascular access site: The Minimizing Adverse Haemorrhagic Events by Transradial Access Site and Systemic Implementation of angioX (MATRIX) trial is the most contemporary and largest trial on access site selection where 8404 ACS patients were randomly allocated to radial or femoral access. ${ }^{43}$ The first co-primary outcome of 30-day MACE - defined as death, MI, or stroke - occurred in $8.8 \%$ of patients with radial access and $10.3 \%$ of patients with femoral access [relative risk (RR) 0.85, 95\% Cl 0.74-0.99; two-sided $P=0.031$; formally nonsignificant at the pre-specified $\alpha$ of 0.025$]$. The second co-primary

\section{Recommendations on $\mathrm{P}_{2} \mathrm{Y}_{12}$ inhibitor selection and timing}

\begin{tabular}{|c|c|c|}
\hline Recommendations & Class $^{\mathrm{a}}$ & Level $^{b}$ \\
\hline $\begin{array}{l}\text { In patients with ACS, ticagrelor ( } 180 \mathrm{mg} \text { loading dose, } 90 \mathrm{mg} \text { twice daily) on top of aspirin }{ }^{c} \text { is recommended, regardless } \\
\text { of initial treatment strategy, including patients pre-treated with clopidogrel (which should be discontinued when ticagre- } \\
\text { lor is commenced) unless there are contraindications. }{ }^{20}\end{array}$ & I & B \\
\hline $\begin{array}{l}\text { In patients with ACS undergoing PCl, prasugrel ( } 60 \mathrm{mg} \text { loading dose, } 10 \mathrm{mg} \text { daily dose) on top of aspirin is recom- } \\
\text { mended for } \mathrm{P}_{2} \mathrm{Y}_{12} \text { inhibitor-naïve patients with NSTE-ACS or initially conservatively managed STEMI if indication for } \\
\mathrm{PCl} \text { is established, or in STEMI patients undergoing immediate coronary catheterization }{ }^{c} \text { unless there is a high risk of } \\
\text { life-threatening bleeding or other contraindications. }{ }^{23}\end{array}$ & I & B \\
\hline $\begin{array}{l}\text { Pre-treatment with a P2Y } 12 \text { inhibitor is generally recommended in patients in whom coronary anatomy is known and } \\
\text { the decision to proceed to PCl is made as well as in patients with STEMI. }{ }^{20,23,38}\end{array}$ & I & A \\
\hline $\begin{array}{l}\text { In patients with NSTE-ACS undergoing invasive management, ticagrelor administration ( } 180 \mathrm{mg} \text { loading dose, } 90 \mathrm{mg} \\
\text { twice daily), or clopidogrel ( } 600 \mathrm{mg} \text { loading dose, } 75 \mathrm{mg} \text { daily dose) if ticagrelor is not an option, should be considered } \\
\text { as soon as the diagnosis is established. }\end{array}$ & Ila & C \\
\hline In patients with stable CAD, pre-treatment with clopidogrel may be considered if the probability of $\mathrm{PCl}$ is high. & IIb & C \\
\hline $\begin{array}{l}\text { Clopidogrel ( } 600 \mathrm{mg} \text { loading dose, } 75 \mathrm{mg} \text { daily dose) on top of aspirin is recommended in stable CAD patients under- } \\
\text { going coronary stent implantation and in ACS patients who cannot receive ticagrelor or prasugrel, including those with } \\
\text { prior intracranial bleeding or indication for OAC. }\end{array}$ & I & A \\
\hline $\begin{array}{l}\text { Clopidogrel ( } 300 \mathrm{mg} \text { loading dose in patients aged } \leq 75,75 \mathrm{mg} \text { daily dose) is recommended on top of aspirin in STEMI } \\
\text { patients receiving thrombolysis. }{ }^{31,32}\end{array}$ & I & A \\
\hline $\begin{array}{l}\text { Ticagrelor or prasugrel on top of aspirin may be considered instead of clopidogrel in stable CAD patients undergoing } \\
\text { PCl, taking into account the ischaemic (e.g. high SYNTAX score, prior stent thrombosis, location and number of } \\
\text { implanted stents) and bleeding (e.g. according to PRECISE-DAPT score) risks. }\end{array}$ & Illb & C \\
\hline In NSTE-ACS patients in whom coronary anatomy is not known, it is not recommended to administer prasugrel. ${ }^{25}$ & III & B \\
\hline
\end{tabular}

ACS = acute coronary syndrome; CAD = coronary artery disease; DAPT = dual antiplatelet therapy; NSTE-ACS = non-ST-elevation acute coronary syndrome; OAC = oral anticoagulant; $\mathrm{PCI}$ = percutaneous coronary intervention; PRECISE-DAPT = PREdicting bleeding Complications In patients undergoing Stent implantation and subsEquent Dual Anti Platelet Therapy; STEMI = ST-elevation myocardial infarction; SYNTAX = Synergy Between Percutaneous Coronary Intervention With Taxus and Cardiac Surgery.

${ }^{\mathrm{a}}$ Class of recommendation.

bevel of evidence.

${ }^{c}$ Contraindications for ticagrelor: previous intracranial haemorrhage or ongoing bleeds. Contraindications for prasugrel: previous intracranial haemorrhage, previous ischaemic stroke or transient ischaemic attack, or ongoing bleeds; prasugrel is not recommended for patients $\geq 75$ years of age or with a body weight $<60 \mathrm{~kg}$. 
outcome of 30-day net adverse clinical events (NACE) [MACE or non-CABG BARC (Bleeding Academic Research Consortium) major bleeding] was experienced in $9.8 \%$ and $11.7 \%$ of patients, respectively (RR 0.83, 95\% Cl 0.73-0.96; $P=0.009$ ). Radial access was associated with a lower risk of all-cause mortality $(1.6 \%$ vs. $2.2 \%$; RR $0.72,95 \% \mathrm{Cl}$ $0.53-0.99 ; P=0.045)$. Major BARC 3 or 5 bleeding was significantly reduced in the radial group (1.6\% vs. $2.3 \%$; RR 0.67, 95\% Cl 0.49-0.92; $P=0.013)$. Radial access was associated with significantly lower rates of surgical access site repair or transfusion of blood products. An updated meta-analysis including MATRIX found a significant reduction in major bleeds; death, MI, or stroke; and in all-cause mortality associated with radial as compared to femoral access. ${ }^{44}$

Aspirin dosing in patients treated with DAPT: Lower aspirin doses ( $\leq 100 \mathrm{mg}$ daily) have been consistently demonstrated to be associated with less major and total bleeding than higher doses, either when used as monotherapy or when combined with the P2Y $Y_{12}$ inhibitor clopidogrel. ${ }^{45-52}$ This is because daily aspirin doses as low as $30-50 \mathrm{mg}$ are able to completely inactivate the platelet cyclooxygenase- 1 enzyme and inhibit thromboxane production. ${ }^{53,54}$ In addition, the efficacy of ticagrelor may be decreased in patients treated with higher aspirin doses ( $\geq 300 \mathrm{mg}$ daily) vs. lower aspirin doses ( $\leq 100 \mathrm{mg}$ daily). ${ }^{55}$ Although the molecular mechanism behind this finding is not entirely clear, it reinforces the use of low dose aspirin. The optimal range of aspirin dose in patients treated with DAPT that provides maximal protection from ischaemic events and minimizes bleeding risk appears to be $75-100 \mathrm{mg}$.

Platelet function testing, genetic testing, and switching of $\mathrm{P}_{2} Y_{12}$ inhibitors: High and low platelet reactivity on $\mathrm{P}_{2} \mathrm{Y}_{12}$ antagonist treatment predicts ischaemic and bleeding risks, respectively. ${ }^{56}$ These data have led to the rationale for individualized antiplatelet therapy based on platelet function monitoring to identify the patients out of the expected range of platelet inhibition. ${ }^{57}$ All randomized trials have failed to demonstrate any benefit of platelet function monitoring to adjust therapy. ${ }^{58-60}$ The low-risk level of the study populations, the exclusive use of clopidogrel, and the $\mathrm{P}_{2} \mathrm{Y}_{12}$ reaction unit thresholds to define the optimal window of $\mathrm{P}_{2} \mathrm{Y}_{12}$ inhibition have been recognized as the main limitations of these trials. ${ }^{61-63}$

The Platelet Function Monitoring to Adjust Antiplatelet Therapy in Elderly Patients Stented for an Acute Coronary Syndrome (ANTARCTIC) trial has re-evaluated the concept of individualized antiplatelet therapy by selecting only ACS patients at high risk of both ischaemic and bleeding events (based on age $\geq 75$ years) and more accurate thresholds in reflecting optimal $\mathrm{P}_{2} \mathrm{Y}_{12}$ inhibition. Clopidogrel was replaced by prasugrel using the recommended daily dose of $5 \mathrm{mg}$ for the elderly, with the possibility of adjustment up and down according to individual response. Platelet function monitoring performed 14 days after discharge and later if needed led to a change of treatment in $45 \%$ of patients who were identified as being overtreated or undertreated by measurement of the P2Y 12 inhibition level; however, this strategy did not improve ischaemic or safety outcomes. ${ }^{64}$ The influence of genetic variants on the response to antiplatelet agents, especially clopidogrel, has been well-established in patients with ACS and planned $\mathrm{PCl} .{ }^{65}$ Rapidly-obtained genetic information on the 2C19 genotype can help in reaching the optimal window of $\mathrm{P}_{2} \mathrm{Y}_{12}$ inhibition according to the cytochrome P450 (CYP) $2 C 19$ profile, ${ }^{66,67}$ but no randomized trial has ever demonstrated any clinical benefit of such an approach. Moreover, only $6-12 \%$ of the variability in on-clopidogrel platelet reactivity can be explained by the differences in genotype. ${ }^{68,69}$

For these reasons, neither platelet function testing nor genetic testing can be recommended for tailoring DAPT. It may be considered in specific situations (e.g. patients suffering from recurrent adverse events) if the results may change the treatment strategy. This is the case for patients undergoing CABG who are exposed to DAPT (see Chapter 5).

Proton pump inhibitors and DAPT: Gastrointestinal haemorrhage is the most common serious bleeding complication from the use of long-term antiplatelet therapy. ${ }^{70} \mathrm{RCT}$ s have shown that PPIs reduce the rate of recurrent gastrointestinal bleeding in high-risk patients receiving aspirin. ${ }^{71}$ Similar data exist regarding the use of famotidine, a histamine $\mathrm{H}_{2}$-receptor antagonist. ${ }^{72}$

Clopidogrel requires metabolic transformation in the liver by CYP isoenzymes (mainly CYP2C19) to elicit its antiplatelet effect. PPls are also metabolized by CYP enzymes, leading to a potential inhibition of CYP2C19 (mainly omeprazole and esomeprazole) translating into reduced metabolic activation of clopidogrel when taken together. Pharmacodynamic studies demonstrated the reduction of clopidogrel-induced antiplatelet effects when a PPI, mainly omeprazole, was concomitantly administered. ${ }^{73-76}$ Based on drug-drug interaction studies, omeprazole and esomeprazole would appear to have the highest propensity for clinically relevant interactions, lansoprazole an intermediate probability, while pantoprazole and rabeprazole have the lowest. ${ }^{77}$ However, importantly, no interaction between concomitant use of PPls and prasugrel or ticagrelor has been observed.

Only observational studies suggested an increased risk of cardiovascular ischaemic events when PPI therapy was administered concomitantly with clopidogrel. ${ }^{78}$ Conversely, randomized trials and propensity score-matched studies did not support such concerns. $^{76,79-81}$

The Clopidogrel and the Optimization of Gastrointestinal Events Trial (COGENT) was a randomized, double-blind, double-dummy, placebo-controlled, phase III study of the efficacy and safety of a fixed-dose combination of clopidogrel $(75 \mathrm{mg})$ and omeprazole (20 mg), as compared with clopidogrel alone. ${ }^{79}$ Patients were eligible if they were 21 years of age or older and if the use of clopidogrel therapy with concomitant aspirin was anticipated for at least the next 12 months, including patients presenting with an ACS or undergoing placement of a coronary stent. Patients at high risk for gastrointestinal bleeding were excluded (i.e. those in whom the need for a PPI, an $\mathrm{H}_{2-}$ receptor antagonist, sucralfate, or misoprostol was anticipated; with pre-existing erosive oesophagitis or oesophageal or gastric variceal disease or previous non-endoscopic gastric surgery; receipt of oral anticoagulation therapy that could not be safely discontinued for the duration of the study; or recent fibrinolytic therapy). Therefore, following previous evidence of benefit from a PPI or $\mathrm{H}_{2}$-receptor antagonist in high-risk patients treated with aspirin monotherapy, the COGENT study included patients at low risk of gastrointestinal bleeding undergoing DAPT, under the rationale that the risk of gastrointestinal bleeding is higher in patients taking aspirin and clopidogrel as compared to aspirin alone. This study was prematurely stopped with a total of 3761 patients instead of the planned 5000 due to financial reasons. The pre-specified primary gastrointestinal efficacy endpoint was the time from randomization to the first occurrence of a composite of upper gastrointestinal clinical events, which 
occurred in $1.1 \%$ of patients with omeprazole and $2.9 \%$ with placebo at 180 days after randomization ( $\mathrm{HR} 0.34,95 \% \mathrm{Cl} 0.18-0.63 ; P<$ $0.001) .^{79}$

Furthermore, there was no significant increase in the risk of cardiovascular events with concomitant use of clopidogrel and omeprazole (4.9\%, 95\% Cl 3.4-6.4\%, in the omeprazole group; and 5.7\%, 95\% Cl $4.0-7.3 \%$, in the placebo group; $P=0.98$ ), a finding that was consistent even in high-risk subgroups and for individual endpoints. The rate of serious adverse events did not differ significantly between the two groups (10.1\% with omeprazole and $9.4 \%$ with placebo, $P=0.48)$, nor did the rate of overall adverse events ( 41.3 and $42.8 \%$, respectively; $P=$ 0.33). Diarrhoea was reported in $3.0 \%$ of patients receiving omeprazole, as compared with $1.8 \%$ of those receiving placebo $(P=0.01)$. There were no newly diagnosed cases of osteoporosis. One case of peripheral neuropathy was reported in the placebo group.

No randomized data comparing use vs. non-use of PPI in patients taking aspirin and prasugrel or ticagrelor exist. However, the risk of gastrointestinal bleeding is higher with DAPT in the form of prasugrel $^{23}$ or ticagrelor ${ }^{82}$ as compared to clopidogrel. The short- and long-term safety profile of PPIs has been well-established..$^{79}$ Impaired magnesium absorption with PPIs has been reported only from studies in which patients had received a PPI for at least 1 year. ${ }^{83}$ Magnesaemia monitoring is recommended at follow-up, especially for longer than 1 year of therapy.

Type, dose of $P 2 Y_{12}$ inhibitor, and duration of treatment: The type and dose of $\mathrm{P}_{2} \mathrm{Y}_{12}$ inhibitor are well-established according to the various settings of CAD. Previous intracranial haemorrhage or ongoing bleeds are common contraindications for prasugrel and ticagrelor, while prasugrel should be given with caution in patients $\geq 75$ years of age or with a body weight $<60 \mathrm{~kg}$. Patients with previous stroke or transient ischaemic attack (TIA) may derive harm from prasugrel instead of clopidogrel. ${ }^{23}$ Prior stroke is a marker of frailty and of subsequent risk of haemorrhagic stroke, especially during the first year thereafter. Switching from prasugrel or ticagrelor to clopidogrel is a common practice, especially in cases of minor bleeding or in patients with low platelet reactivity, a marker of major bleeding risk. ${ }^{56,84,85}$ There are no properly powered randomized data on the long-term safety or efficacy of 'switching' patients treated for weeks or months with a P2Y 12 inhibitor to a different P2Y 12 inhibitor. Therefore, this practice is generally discouraged.

\subsection{Switching between oral ${\mathrm{P} 2 \mathrm{Y}_{12}}_{12}$ inhibitors}

Differences in the pharmacology of $\mathrm{P}_{2} \mathrm{Y}_{12}$ receptor inhibitors with regard to their binding site, half-life, and speed of onset and offset of action are important factors that might lead to drug interactions when switching from one agent to another.

The transition from clopidogrel to ticagrelor is the only switch between P2Y $_{12}$ inhibitors that has been investigated in a trial powered for clinical endpoint, even if the study was not specifically designed to assess the safety and efficacy of the transition from clopidogrel to ticagrelor. In PLATO, nearly $50 \%$ of patients randomly allocated to receive ticagrelor had been pre-treated with clopidogrel, mostly given as a $300-600 \mathrm{mg}$ loading dose. ${ }^{20}$ The efficacy and safety of ticagrelor were not affected by previous clopidogrel exposure. ${ }^{88}$ On the other hand, the TRITON-TIMI 38 trial mandated that previous exposure of patients to a $\mathrm{P}_{2} \mathrm{Y}_{12}$ receptor inhibitor

\section{Switching between oral $\mathrm{P}_{2} \mathrm{Y}_{12}$ inhibitors}

\begin{tabular}{|c|c|c|}
\hline Recommendations & Class $^{\mathrm{a}}$ & Level $^{\mathrm{b}}$ \\
\hline $\begin{array}{l}\text { In patients with ACS who were previously } \\
\text { exposed to clopidogrel, switching from clopi- } \\
\text { dogrel to ticagrelor is recommended early } \\
\text { after hospital admission at a loading dose of } \\
180 \mathrm{mg} \text { irrespective of timing and loading } \\
\text { dose }^{c} \text { of clopidogrel, unless contraindications } \\
\text { to ticagrelor exist. }^{20}\end{array}$ & I & B \\
\hline $\begin{array}{l}\text { Additional switching between oral } \mathrm{P}_{2} \mathrm{Y}_{12} \\
\text { inhibitors may be considered in cases of } \\
\text { side effects/drug intolerance according to } \\
\text { the proposed algorithms. }\end{array}$ & Illb & C \\
\hline $\begin{array}{l}\text { S = acute coronary syndrome. } \\
\text { ass of recommendation. } \\
\text { evel of evidence. } \\
\text { ontraindications for ticagrelor: previous intracrani } \\
\text { eds. }\end{array}$ & emorrha & or ongoing \\
\hline
\end{tabular}

\section{Measures to minimize bleeding while on dual antiplatelet therapy}

\begin{tabular}{|c|c|c|}
\hline Recommendations & Class $^{\mathrm{a}}$ & Level $^{\mathrm{b}}$ \\
\hline Radial over femoral access is recommended for coronary angiography and PCl if performed by an expert radial operator. ${ }^{43,44}$ & I & $\mathbf{A}$ \\
\hline In patients treated with DAPT, a daily aspirin dose of $75-100 \mathrm{mg}$ is recommended. ${ }^{45-47,51,52}$ & $\mathbf{I}$ & $\mathbf{A}$ \\
\hline A PPI in combination with DAPT ${ }^{c}$ is recommended. ${ }^{70,79,80,86,87}$ & $\mathbf{I}$ & B \\
\hline Routine platelet function testing to adjust antiplatelet therapy before or after elective stenting is not recommended. ${ }^{58-60}$ & III & A \\
\hline
\end{tabular}

DAPT = dual antiplatelet therapy; $\mathrm{PCl}=$ percutaneous coronary intervention; PPI proton pump inhibitor.

${ }^{\mathrm{a}}$ Class of recommendation.

bLevel of evidence.

'While the evidence that a PPI does not increase the risk of cardiovascular events was generated with omeprazole, based on drug-drug interaction studies, omeprazole and esomeprazole would appear to have the highest propensity for clinically relevant interactions, while pantoprazole and rabeprazole have the lowest. 
should be an exclusion criterion for study entry. ${ }^{23}$ While registry data provide reassuring information with respect to the safety profile of switching from clopidogrel to prasugrel, ${ }^{89-91}$ no randomized data exist in the setting of studies powered for clinical endpoint. Similarly, all other switching possibilities, including between prasugrel and ticagrelor or from ticagrelor/prasugrel to clopidogrel, have not been investigated with outcome data. ${ }^{92-94}$ This practice is therefore discouraged due to a lack of safety/efficacy data. As the need to switch between $\mathrm{P}_{2} \mathrm{Y}_{12}$ inhibitors may arise for clinical reasons (i.e. side effects or drug intolerance), and registry data indicate that switching is not infrequent in practice, switching algorithms based on pharmacodynamic studies are provided (Figure 2).

\section{Dual antiplatelet therapy and percutaneous coronary intervention}

An overview of all studies investigating the benefits and risks of DAPT duration beyond 1 month, largely focusing on post$\mathrm{PCl}$ patients or those with prior ACS, is shown in Web addenda Table S1 (A and B). An overview of recommendations endorsed by these guidelines regarding DAPT duration after $\mathrm{PCl}$, as well as after CABG or in medically managed ACS patients, is provided in Figure 3.

\subsection{Dual antiplatelet therapy after percutaneous coronary intervention for stable coronary artery disease}

DAPT is not indicated in purely medically managed patients (i.e. without prior $\mathrm{PCl}$ ) with stable CAD and no history of prior MI. The Clopidogrel for High Atherothrombotic Risk and Ischemic Stabilization, Management, and Avoidance (CHARISMA) study included patients with stable vascular disease or at risk of atherothrombotic events, and showed that clopidogrel plus aspirin was not significantly more effective than aspirin alone in reducing the rate of MI, stroke, or death from cardiovascular causes. ${ }^{95}$

After PCl with stent placement, DAPT is the standard of care. The Intracoronary Stenting and Antithrombotic Regimen (ISAR) trial ${ }^{2}$ and, subsequently, other studies ${ }^{96,97}$ established the 1-month course of DAPT after bare-metal stent (BMS). An arbitrary 12-month or more DAPT duration has been subsequently recommended based on expert opinions after first generation DES, irrespective of clinical presentation.

No dedicated study exists focusing on stable CAD patients undergoing $\mathrm{PCl}$ and being exposed to different DAPT durations. Hence, recommendations regarding stable $\mathrm{CAD}$ patients undergoing $\mathrm{PCl}$ derive from subgroup analyses from pertinent RCTs (Figure 4). 98,99

While no RCTs investigating the use of ticagrelor or prasugrel instead of clopidogrel in stable CAD patients undergoing PCl exist, this treatment option may be considered in selected patients in whom the use of clopidogrel is unsatisfactory based on prior clinical outcomes or potentially associated with higher risk of ischaemic events that bleeding recurrences.

Three- or 6- vs. at least 12-month DAPT duration: The Efficacy of Xience/Promus Versus Cypher to Reduce Late Loss After
Stenting (EXCELLENT) trial compared a 6-month DAPT [acetylsalicylic acid (ASA) + clopidogrel] duration with 1-year DAPT after DES. ${ }^{100}$ With 1443 patients randomized, the rates of target vessel failure - defined as the composite of cardiac death, MI, or ischaemia-driven target vessel revascularization-at 12 months were $4.8 \%$ in the 6 -month DAPT group and $4.3 \%$ in the 12 -month DAPT group ( $P=0.001$ for non-inferiority). There was a numerically lower risk of bleeding in the short DAPT arm (HR 0.50, 95\% Cl 0.09-2.73). There was no signal of heterogeneity for the primary study endpoint with respect to clinical presentation (i.e. stable CAD, $n=699$ patients vs. ACS, $n=744$ patients). The PROlonging Dual antiplatelet treatment after Grading stentinduced intimal hYperplasia (PRODIGY) trial randomized 2013 patients $^{101}$ to 6 or 24 months of DAPT (ASA + clopidogrel) and to one of four stent types (a four-by-two factorial design), including BMS and three different DES types. The 2-year incidence of allcause death, MI, and stroke or cerebrovascular accident was $10.1 \%$ with 24 -month DAPT compared with $10.0 \%$ with 6-month DAPT $(P=0.91)$. There was a lower risk of major bleeding with shorter DAPT based on both the BARC (1.9\% vs. 3.4\%; HR 0.56, $95 \% \mathrm{Cl} 0.32-0.98 ; P=0.037)$ or the TIMI scale $(0.6 \%$ vs. $1.6 \%$; HR $0.38,95 \% \mathrm{Cl} 0.15-0.97 ; P=0.041)$. After censoring events that occurred after 12 months, while keeping the original randomization design, the risk of TIMI major bleeding was $0.5 \%$ in the shortterm DAPT arm vs. $0.9 \%$ in the long-term DAPT arm (HR 0.56, 95\% Cl 0.19-1.66). In this trial, a total of 1465 (74.3\%) patients presented with ACS whereas 505 (25.7\%) had stable CAD. ${ }^{99}$ No heterogeneity was noted with respect to the primary efficacy endpoint. There was a borderline quantitative interaction between clinical presentations and bleeding outcomes ( $P$ values for interaction $=0.056$ for BARC 2, 3, or $5 ; P=0.091$ for BARC 3 or 5 ), suggesting a higher hazard of bleeding in the 24-month DAPT arm when compared with the 6-month arm in the stable CAD patients, which was not observed in the ACS patients. ${ }^{99}$ Analysis of NACE-consisting of death, MI, cerebrovascular accident, or BARC 2, 3, or 5 bleeding-revealed significant harm from extended DAPT in stable CAD patients (NACE in the 24-month vs. 6-month DAPT arm: $13.3 \%$ vs. $5.6 \%$; HR 2.5, 95\% Cl 1.35-4.69, $P=0.004$; NNT for harm $=13$ ) and no benefit in the ACS population (16.1\% vs. $14.1 \%$; HR 1.15, 95\% Cl 0.88-1.50; $P=0.29)$, with positive quantitative interaction testing $(P$ value for interaction $=$ 0.024). ${ }^{99}$ Patients with high CRUSADE (Can Rapid risk stratification of Unstable angina patients Suppress ADverse outcomes with Early implementation of the ACC/AHA Guidelines) bleeding risk score treated with 24-month DAPT experienced a threefold higher risk of major bleeding and a fivefold risk of red blood cell transfusion as compared with 6-month therapy, without clear evidence of benefit. $^{13}$

In 2014, three additional randomized studies were published that compared 6 months of DAPT to 12 or 24 months of DAPT (ASA + clopidogrel): Is There a Life for DES After Discontinuation of Clopidogrel (ITALIC), ${ }^{102}$ Second Generation Drug-Eluting Stent Implantation Followed by Six- Versus TwelveMonth Dual Antiplatelet Therapy (SECURITY), ${ }^{103}$ and Intracoronary Stenting and Antithrombotic Regimen: Safety and Efficacy of 6 Months Dual Antiplatelet Therapy After Drug-Eluting 

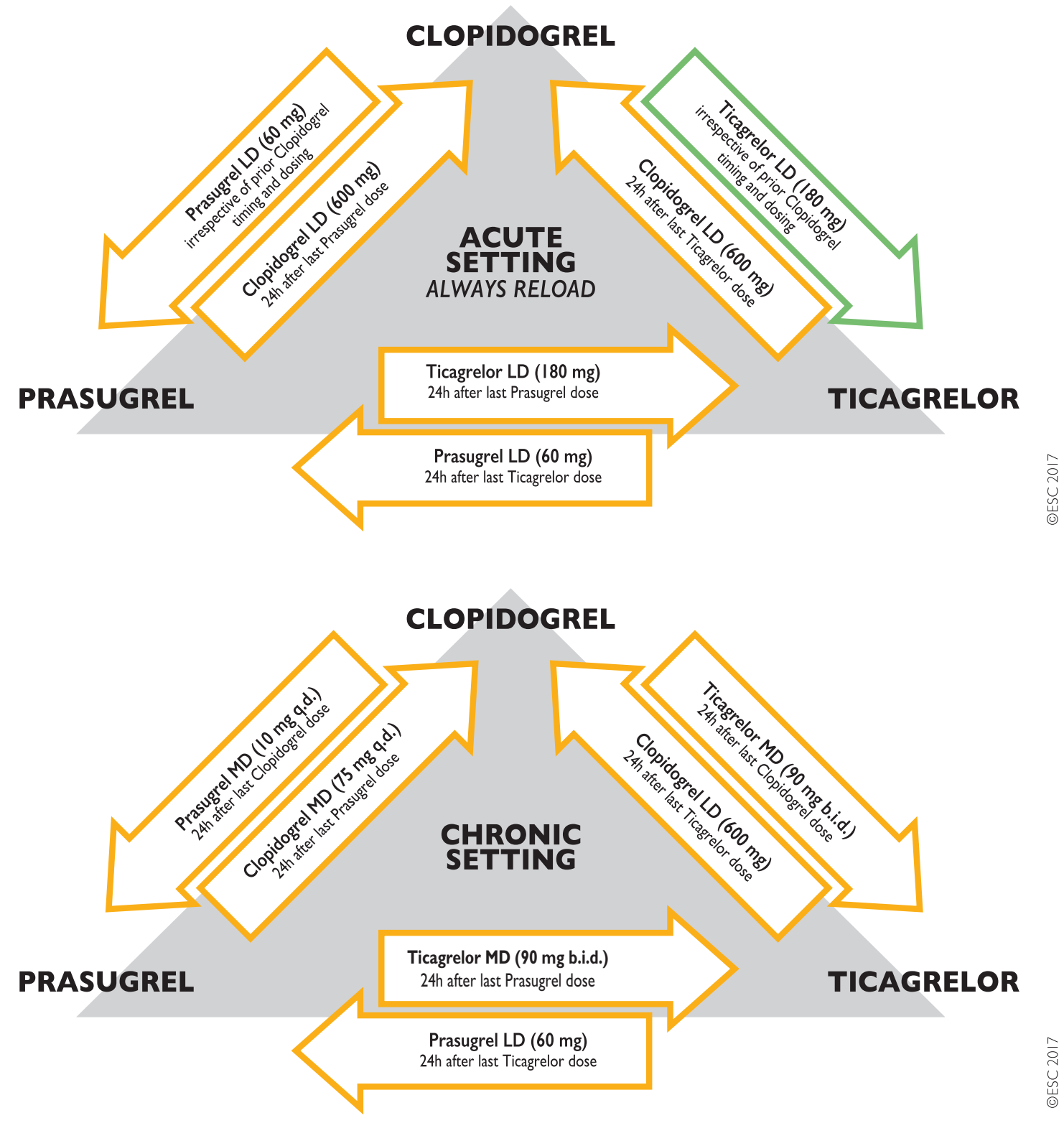

Figure 2 Algorithm for switching between oral P2Y 12 inhibitors in the acute and chronic setting. LD = loading dose; $M D=$ maintenance dose. Colour-coding refers to the ESC Classes of Recommendations (green = Class l; orange = Class llb). The green arrow from clopidogrel to ticagrelor highlights the only switching algorithm for which outcome data are available in patients with acute coronary syndrome. No outcome data (orange arrows) are available for all other switching algorithms. Acute setting is considered as a switching occurring during hospitalization.

Stenting (ISAR-SAFE). ${ }^{104}$ ISAR-SAFE was the largest of these three studies, with 4005 randomized patients, and the only double-blind investigation. It confirmed that a 12-month course of DAPT did not afford any benefit over a 6-month course with respect to ischaemic endpoints. Likewise, the net clinical benefit (composite of death, MI, stent thrombosis, stroke, and TIMI major bleeding) was neutral. At subgroup analysis, there was no signal of heterogeneity with respect to the primary study endpoint among the 2394 patients who presented with stable CAD as opposed to the 1601 patients with ACS. ${ }^{104}$ Consistent results were shown in the ITALIC and SECURITY trials. Two studies, Real Safety and Efficacy of 3-Month Dual Antiplatelet Therapy Following Endeavor Zotarolimus-Eluting Stent Implantation (RESET) ${ }^{105}$ and Optimized Duration of Clopidogrel Therapy Following Treatment With the Zotarolimus-Eluting Stent in Real-World Clinical Practice (OPTIMIZE), ${ }^{106}$ investigated a 3-month duration of DAPT (ASA + clopidogrel). RESET randomized 2117 patients to 3- or 12-month duration of DAPT and did not show significant 

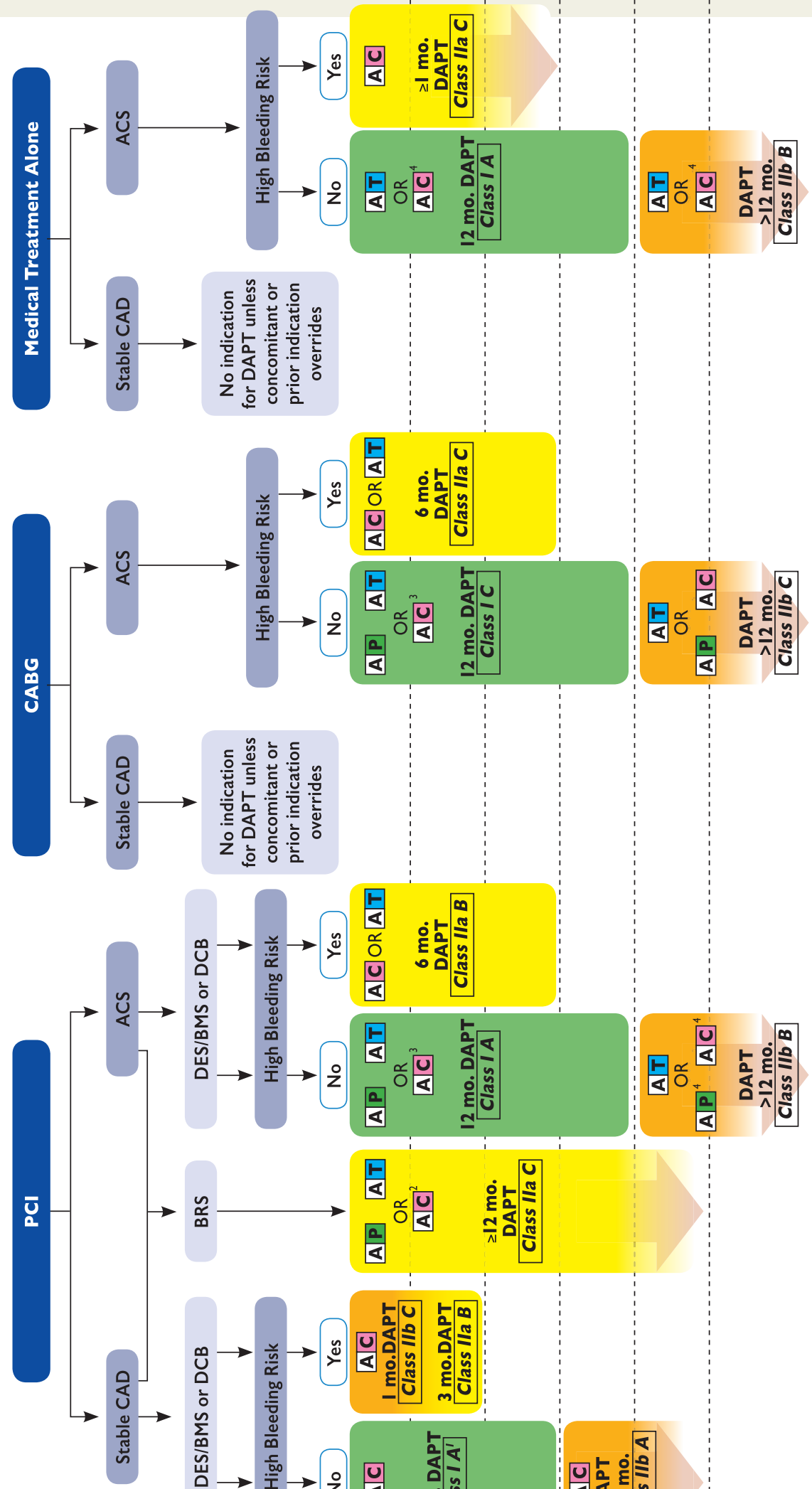

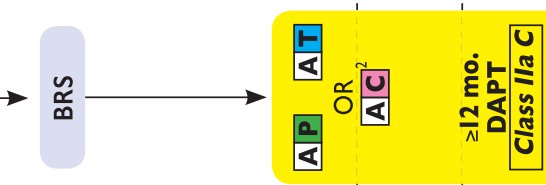
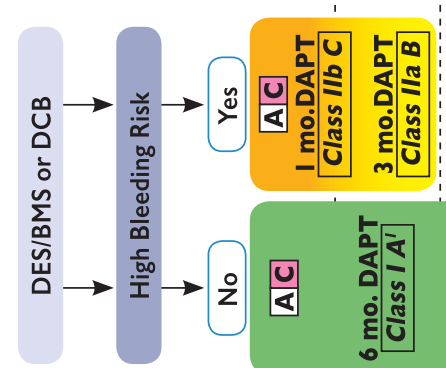

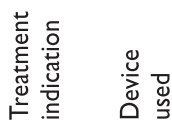
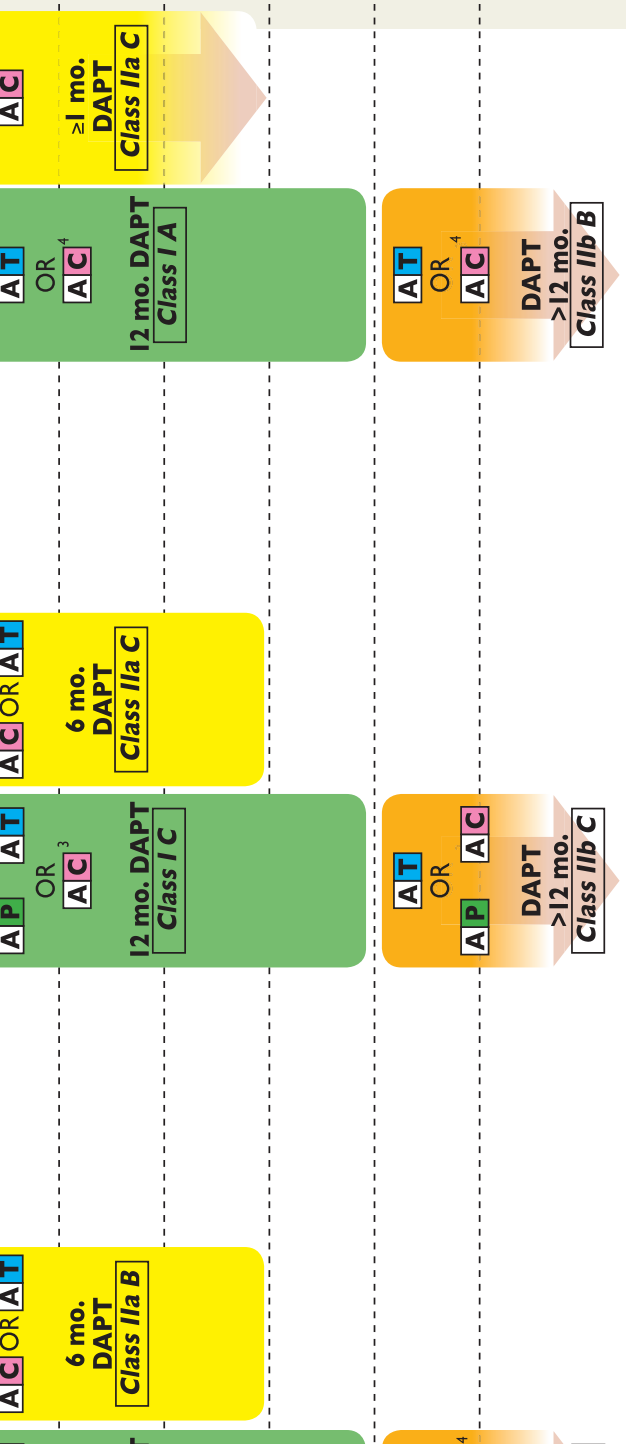


\section{Percutaneous Coronary Intervention}

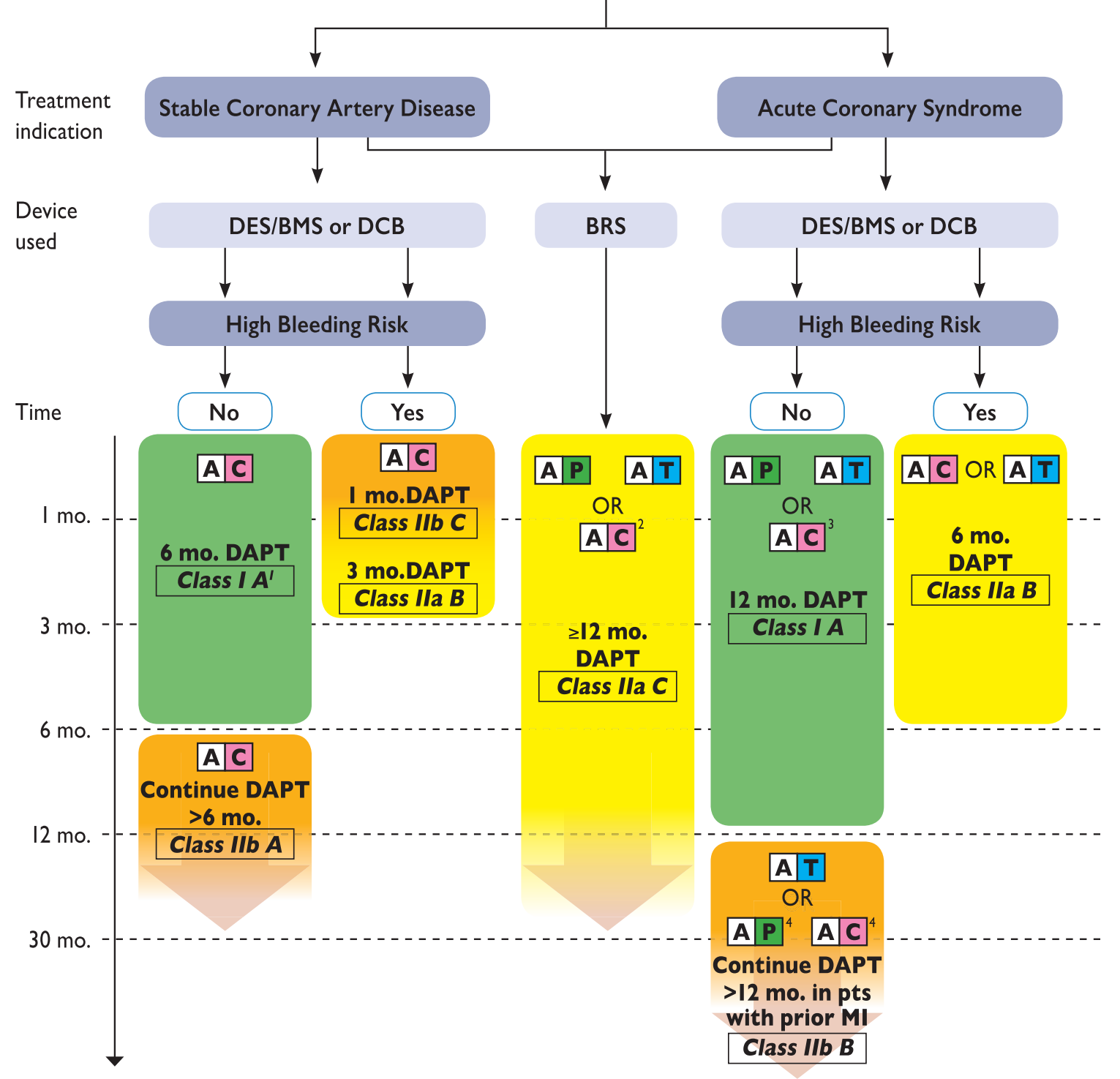

$\mathbf{A}=$ Aspirin $\quad \mathbf{C}=$ Clopidogrel $\quad \mathbf{P}=$ Prasugrel $\quad \mathbf{T}=$ Ticagrelor

Figure 4 Algorithm for dual antiplatelet therapy (DAPT) in patients treated with percutaneous coronary intervention. ACS = acute coronary syndrome; BMS = bare-metal stent; BRS = bioresorbable vascular scaffold; CABG = coronary artery bypass graft surgery; DCB = drug-coated balloon; DES: drug-eluting stent; $\mathrm{PCl}=$ percutaneous coronary intervention; Stable $\mathrm{CAD}=$ stable coronary artery disease.

High bleeding risk is considered as an increased risk of spontaneous bleeding during DAPT (e.g. PRECISE-DAPT score $\geq 25$ ).

Colour-coding refers to the ESC Classes of Recommendations (green = Class I; yellow = lla; orange = Class Ilb).

Treatments presented within the same line are sorted in alphabetic order, no preferential recommendation unless clearly stated otherwise.

1: After PCl with DCB 6 months. DAPT should be considered (Class lla B).

2: If patient presents with Stable CAD or, in case of $A C S$, is not eligible for a treatment with prasugrel or ticagrelor.

3: If patient is not eligible for a treatment with prasugrel or ticagrelor.

${ }^{4}$ : If patient is not eligible for a treatment with ticagrelor. 
harm with the shortened period (composite rates of any death, $\mathrm{MI}$, or stent thrombosis $0.8 \%$ vs. $1.3 \% ; P=0.48)$. Similar results were achieved in OPTIMIZE with 3119 patients randomized. In this study, the 1-year incidence of MACE was $8.3 \%$ in the shortterm group and $7.4 \%$ in the long-term group $(\mathrm{HR} 1.12,95 \% \mathrm{Cl}$ 0.87-1.45). Both studies mandated the use of the Endeavor zotarolimus-eluting stent (ZES) in the 3-month DAPT arms, which is no longer available on the market. It is not clear to what extent the results of RESET and OPTIMIZE are applicable to other types of DES.

Palmerini et al performed a meta-analysis addressing the outcome of a $\leq 6$-month course of DAPT vs. a 1-year course after DES. ${ }^{107}$ The 1 -year course of therapy did not confer any advantage over the shorter course of DAPT with respect to survival, stent thrombosis, or MI, but it increased the risk of major bleeding substantially. Similar results were obtained by other meta-analyses. ${ }^{108,109}$

Twelve-month vs. >12-month DAPT duration: Following the proposed landmark of 12 months as the standard DAPT duration after DES, the DAPT trial investigated whether further extension of DAPT might be beneficial. ${ }^{110}$ The DAPT study enrolled patients who, at 12 months after placement of a DES, were still on DAPT and had not suffered an ischaemic or bleeding event. Patients were randomly allocated to thienopyridine or placebo for another 18 months. Aspirin was maintained throughout the study period. Thirty-month DAPT as compared with 12-month DAPT reduced the rates of stent thrombosis $(0.4 \%$ vs. $1.4 \% ; P<0.001)$ and of major adverse cardiac and cerebrovascular events (MACCE) (4.3\% vs. 5.9\%; $P<0.001)$. This included a substantial reduction in the rate of $\mathrm{MI}(2.1 \%$ vs. $4.1 \%$; $P<0.001)$; slightly more than half of this benefit could be attributed to the prevention of spontaneous Mls (see chapter 3.2). This ischaemic protection came at the cost of an increased risk of bleeding (GUSTO moderate or severe bleeding $2.5 \%$ vs. $1.6 \%, P<0.001$ ) and an increase in total mortality with borderline statistical significance (see section 3.3).

Of 11648 randomized patients within the DAPT trial (9961 treated with DES and 1687 with BMS), $30.7 \%$ presented with MI. ${ }^{98}$ The excess of mortality observed within the 30-month DAPT arm was entirely driven by fatalities, which occurred in patients without prior $\mathrm{MI}$ (2.1\% for continued thienopyridine group vs. $1.5 \%$ for placebo; HR 1.43, 95\% Cl 1.02-2.00; $P=0.04$ ). Yet, the interaction $P$ value did not reach statistical significance (effect for $M I$ vs. no MI interaction $P=0.13) .{ }^{99}$

Three independent meta-analyses, which included 5045 patients recruited within DES-Late coronary Arterial Thrombotic Events (LATE) $)^{111}$ and 1259 patients from the Assessment by a Double Randomisation of a Conventional Antiplatelet Strategy Versus a Monitoring-Guided Strategy for Drug-Eluting Stent Implantation and, of Treatment Interruption Versus Continuation 1 Year After Stenting-Interruption (ARCTIC-Interruption) trial, $^{112}$ provided results consistent with a possible increase in mortality with prolonged DAPT as shown in the DAPT trial. A more recent metaanalysis of 11 RCTs that enrolled 33051 patients who received predominantly newer-generation DES also provided weak evidence of an increased mortality rate with prolonged DAPT. ${ }^{113}$

Thus, if DAPT is administered for a sufficient length of time after placement of DES for stable CAD, a substantial benefit in terms of secondary prevention and reduction of stent thrombosis emerges. However, this benefit is counterbalanced by an increased risk of bleeding and by a signal for increased mortality. Thus, systematic extension of DAPT beyond six months is not justified for all patients but should be based on the individual risk profile of the patient (see section 3.5).

Impact of type of DES on duration of DAPT: The benefit of extended periods of DAPT varies with stent type. However, there are differences between first- and newer-generation DES. In PRODIGY, only patients with the paclitaxel-eluting stent benefitted from extended DAPT with a significant reduction of the risk of stent thrombosis. ${ }^{114}$ Likewise, in DAPT, the benefit of extended DAPT was largest with patients with a paclitaxel-eluting stent and the smallest with an everolimus-eluting stent. ${ }^{110,115}$ There also was a significant interaction between stent type and benefit of extended DAPT with respect to MACCE. ${ }^{110}$ With an everolimuseluting stent, the 1-year NNT for prevention of stent thrombosis was 157, whereas the 1-year NNT for harm for moderate or severe bleeding was $56 .{ }^{115}$ In the meta-analysis by Giustino et al, ${ }^{109}$ the reduction of stent thrombosis by extended DAPT was significantly reduced with new-generation stents as compared with firstgeneration DES, and statistical significance was lost within the new-generation subset. No such interaction was found concerning bleeding complications. Similar results were obtained in two other meta-analyses (Sharma et al ${ }^{116}$ and Palmerini et al ${ }^{117}$ ).

Bioresorbable stents and drug-coated balloons: No dedicated studies examining the optimal duration of DAPT after implantation of a bioresorbable scaffold currently exist. In the largest randomized clinical trial investigating the treatment of patients with a poly-lactic acid-based bioresorbable scaffold, DAPT was recommended for at least 12 months. ${ }^{118}$ However, meta-analysis has shown evidence of an approximately twofold higher rate of stent thrombosis in comparison with conventional DES, especially in the first 30 days after implantation. ${ }^{119}$ This provides a rationale for considering more potent $\mathrm{P}_{2} \mathrm{Y}_{12}$ inhibitors in these patients. In addition, some concerns have been raised regarding late stent thrombosis beyond 1 year after implantation ${ }^{120,121}$ and a longer duration of DAPT therapy may be advocated, at least in patients at low bleeding risk. No large-scale clinical trials are available concerning magnesiumbased bioresorbable scaffolds.

In patients treated with drug-coated balloons, dedicated clinical trials investigating the optimal duration of DAPT are lacking. In patients treated for in-stent restenosis, the largest randomized trials investigating drug-coated balloon therapy have recommended a treatment duration of between 3-12 months. ${ }^{122-124}$ In addition, some small clinical trials, as well as larger registries, including patients with stable CAD undergoing drug-coated balloon angioplasty have recommended DAPT duration of at least 1 month. ${ }^{125}$

Plain old balloon angioplasty: no data on DAPT or DAPT duration exist after plain old balloon angioplasty, which is currently reserved for a small minority of patients in whom stent implantation is not feasible (e.g. small calibre vessel or extreme vessel tortuosity) or desirable (e.g. to avoid DAPT in patients referred to CABG). The institution of DAPT and its duration, if implemented, should depend on clinical profile (ischaemic vs. bleeding risks) and/or the reasons (e.g. planned surgery) for avoiding stent implantation. 
Dual antiplatelet therapy duration and related stent choices in patients with stable coronary artery disease treated with percutaneous coronary intervention

\begin{tabular}{|c|c|c|}
\hline Recommendations & Class $^{a}$ & Level $^{\mathrm{b}}$ \\
\hline $\begin{array}{l}\text { In patients with stable CAD treated with } \\
\text { coronary stent implantation, DAPT consist- } \\
\text { ing of clopidogrel in addition to aspirin is } \\
\text { generally recommended }{ }^{c} \text { for } 6 \text { months, irre- } \\
\text { spective of the stent type. }{ }^{100,101,104,126-130}\end{array}$ & I & A \\
\hline $\begin{array}{l}\text { Irrespective of the intended DAPT duration, } \\
D^{c} S^{c} \text { is the preferred treatment option. }{ }^{129-132}\end{array}$ & I & A \\
\hline $\begin{array}{l}\text { In patients with stable CAD considered at high } \\
\text { bleeding risk (e.g. PRECISE-DAPT } \geq 25 \text { ), DAPT } \\
\text { for } 3 \text { months }{ }^{\text {d }} \text { should be considered. }{ }^{105,106}\end{array}$ & Ila & B \\
\hline $\begin{array}{l}\text { In patients with stable CAD treated with } \\
\text { drug-coated balloon, DAPT for } 6 \text { months } \\
\text { should be considered. }\end{array}$ & Ila & B \\
\hline $\begin{array}{l}\text { In patients with stable CAD treated with } \\
\text { bioresorbable vascular scaffolds, DAPT for } \\
\text { at least } 12 \text { months should be considered. }\end{array}$ & Ila & C \\
\hline $\begin{array}{l}\text { In patients with stable CAD who have toler- } \\
\text { ated DAPT without a bleeding complication } \\
\text { and who are at low bleeding but high throm- } \\
\text { botic risk, continuation of DAPT with clopi- } \\
\text { dogrel for }>6 \text { months and } \leq 30 \text { months may } \\
\text { be considered. }{ }^{26,107-109}\end{array}$ & Illb & A \\
\hline $\begin{array}{l}\text { In patients with stable CAD in whom 3- } \\
\text { month DAPT poses safety concerns, DAPT } \\
\text { for } 1 \text { month }{ }^{\mathrm{e}} \text { may be considered. }\end{array}$ & Illb & C \\
\hline
\end{tabular}

BMS = bare-metal stent; $C A D=$ coronary artery disease; DAPT = dual antiplatelet therapy; DES = drug-eluting stent; $\mathrm{MI}=$ myocardial infarction; PRECISE-DAPT $=$ PREdicting bleeding Complications In patients undergoing Stent implantation and subsEquent Dual Anti Platelet Therapy.

${ }^{a}$ Class of recommendation.

bLevel of evidence.

'These recommendations refer to stents that are supported by large-scale randomized trials with clinical endpoint evaluation leading to unconditional CE mark, as detailed in Byrne et al. ${ }^{134}$

${ }^{\mathrm{d}}$ The evidence supporting this recommendation comes from two studies where zotarolimus-eluting Endeavour sprint stent has been investigated in conjunction with a 3-month DAPT regimen.

e1-month DAPT following implantation of zotarolimus-eluting Endeavour sprint stent or Biofreedom drug-coated stent reduced risks of re-intervention, myocardial infarction and inconsistently of stent thrombosis compared to bare-metal stent under similar DAPT duration. ${ }^{129,130}$ It is unclear if this evidence applies to other contemporary DES.

\subsection{Dual antiplatelet therapy after percutaneous coronary intervention for acute coronary syndrome}

DAPT with novel P2Y 12 inhibitors for 1 year after PCI for ACS: The evidence supporting the value of the combination of aspirin and clopidogrel in patients with ACS has been extensively reviewed in previous guidelines (NSTE-ACS), and data supporting the superiority of ticagrelor and prasugrel over clopidogrel in this setting are discussed in section 3.6.

Although both prasugrel and ticagrelor significantly increase the risk of TIMI major non-CABG related bleeds, the risk-benefit ratios were favourable with NNT for benefit of 46 and 53, respectively, and NNT for harm of 167 for both agents. These data established the 1year course of DAPT, preferably with prasugrel or ticagrelor, for patients undergoing $\mathrm{PCl}$ for $\mathrm{ACS}$, unless there are contraindications (Figure 4).

Mounting evidence for secondary prevention by intensified antiplatelet therapy: In patients presenting with ACS, the cardiovascular risk remains substantially elevated beyond the first year, even if successful revascularization has been achieved. In this setting, intensified antiplatelet therapy on top of aspirin has been shown to be an effective therapeutic strategy to prevent recurrent ischaemic events. However, the risk-benefit ratios seem less favourable than those observed in studies assessing $\leq 1$-year DAPT duration. Relevant information has been provided by the prior Ml patient subsets included in the CHARISMA ${ }^{135}(n=3846)$ and DAPT $^{98}(n=3576)$ trials, which mainly compared clopidogrel with placebo on top of aspirin; by the subset of patients who underwent coronary angiography within the TRILOGY ${ }^{136}$ trial, which compared prasugrel with clopidogrel; and by the patients with prior MI within the Thrombin Receptor Antagonist in Secondary Prevention of Atherothrombotic Ischemic Events (TRA 2 ${ }^{\circ}$ P-TIMI 50) ${ }^{137}(n=17779)$ trial, which compared vorapaxar with placebo. Taken separately, these trial results are difficult to interpret because they are based on subgroup analyses. Moreover, CHARISMA and TRILOGY had a neutral main outcome and the main results of TRA $2^{\circ} \mathrm{P}-\mathrm{TIMI} 50$ showed an unfavourable risk-benefit ratio. Therefore, a dedicated trial on prolonged DAPT for secondary prevention after ACS was needed. The PEGASUS trial filled this gap. ${ }^{29}$

DAPT with ticagrelor for secondary prevention after MI: PEGASUS recruited 21162 patients with spontaneous MI 1-3years before enrolment, who were at $\geq 50$ years old and had at least one additional high-risk feature: age $\geq 65$ years, diabetes mellitus, a second spontaneous MI, multivessel CAD, or chronic renal dysfunction. ${ }^{29}$ The patients were randomly assigned to ticagrelor $90 \mathrm{mg}$ b.i.d., ticagrelor $60 \mathrm{mg}$ b.i.d., or placebo. All the patients received low-dose aspirin. Of the patients included in PEGASUS, 53\% were enrolled after a STEMI and $83 \%$ were previously treated by $\mathrm{PCl}$. The primary efficacy endpoint was the composite of cardiovascular death, MI, or stroke at 3 years and was $7.85 \%$ in the $90 \mathrm{mg}$ arm, $7.77 \%$ in the $60 \mathrm{mg}$ arm, and $9.04 \%$ in the placebo arm $(P=0.008$ and $P=0.004$ for the higher and lower doses, respectively, vs. placebo). ${ }^{29}$ There was a consistent reduction in all components of the primary endpoint with ticagrelor vs. placebo, which reached statistical significance for $\mathrm{MI}$ with both doses of ticagrelor and for stroke with the lower dose. There was also a trend for a reduction in cardiovascular mortality. Due to a non-significant yet numerical increase in non-cardiovascular deaths in the two ticagrelor arms, the outcome was neutral with respect to all-cause death. The primary safety endpoint of TIMI major bleeding was observed more frequently with ticagrelor ( $2.60 \%$ with $90 \mathrm{mg}$ and $2.30 \%$ with $60 \mathrm{mg}$ ) than with placebo (1.06\%) ( $P<0.001$ for each dose vs. placebo). The NNT for benefit for the primary endpoint was 250 for the $90 \mathrm{mg}$ 
dose and 238 for the $60 \mathrm{mg}$ dose; the corresponding NNT for harm was 244 and 322, respectively, with the two ticagrelor doses. ${ }^{29}$

With the $90 \mathrm{mg}$ dose, the absolute benefit in terms of the primary efficacy endpoint was in the same order as the absolute harm in terms of the primary safety endpoint, and with $60 \mathrm{mg}$ the absolute benefit was only marginally larger than the absolute harm. However, the relevance of the various endpoints to the patient's overall wellbeing may differ and are, therefore, difficult to weigh against one another. The impact of $\mathrm{Ml}$ and bleeding on mortality was comparable in previous studies. ${ }^{11,138}$ A post hoc analysis from the Thrombin Receptor Antagonist for Clinical Event Reduction in Acute Coronary Syndrome (TRACER) trial suggested that while bleeding according to BARC 2 and 3 a criteria was less prognostic for death than MI, the risk of mortality was equivalent between BARC 3 b bleeding and $\mathrm{Ml}$, and was higher following BARC $3 \mathrm{c}$ bleeding. Moreover, at variance with previous analyses, both $\mathrm{Ml}$ and bleeding impacted mortality with similar time dependency. ${ }^{42}$ In view of these consistent findings throughout multiple independent studies, both the efficacy and the safety endpoints deserve attention, as both most likely similarly impact mortality. With this background, the narrow risk-benefit ratio cautions against the universal long-term administration of ticagrelor for secondary prevention after $\mathrm{Ml}$, and calls for individualized treatment decisions based on ischaemic and haemorrhagic risk.

To this end, patients who continued their thienopyridine treatment without (a major) interruption ( $\leq 30$ days) derived a larger benefit from extended ticagrelor intake than patients who interrupted their thienopyridine treatment for longer periods of time. ${ }^{139}$ Depending on the actual discontinuation time frame of previous thienopyridine therapy, the HRs $(95 \% \mathrm{Cl})$ of the primary endpoint for ticagrelor (pooled doses) vs. placebo were 0.73 (0.61-0.87) for those who continued within 30 days, 0.86 (0.71-1.04) for those who interrupted for 30 days to 1 year, and 1.01 (0.80-1.27) for those who interrupted for more than 1 year $(P$ trend for interaction $<0.001) .{ }^{139}$ There was no significant interaction of timing with the effect of ticagrelor on bleeding risk. These findings suggest that patients who can continue their initial thienopyridine treatment are those deriving relatively greater benefit from DAPT continuation with ticagrelor. Nevertheless, even in this patient subset, the absolute increase in TIMI major bleeding associated with extended ticagrelor was similar in magnitude as compared to the absolute decrease in the composite ischaemic endpoint (i.e. 1.9 percentage point difference for both the safety and the efficacy endpoints). ${ }^{139}$

Patients with lower-extremities artery disease (LEAD), who are known to be at greater ischaemic risk, also derived heightened benefit from extended ticagrelor. ${ }^{140}$ In these patients, the absolute decreases in the primary efficacy endpoint achieved by ticagrelor vs. placebo were $3.0 \%$ for the $90 \mathrm{mg}$ dose and $5.2 \%$ for the $60 \mathrm{mg}$ dose, whereas the increases in TIMI major bleeding were only $0.22 \%$ and $0.02 \%$, respectively. In addition, ticagrelor was significantly associated with fewer events related to LEAD (i.e. acute limb ischaemia and peripheral revascularization procedures).

DAPT with thienopyridines (clopidogrel or prasugrel) for secondary prevention after Ml: In the DAPT trial, 3567 patients had initially presented with $\mathrm{MI}^{98} \mathrm{~A}$ non-prespecified analysis of these patients investigated whether the benefits and risks of extended vs. standard duration of DAPT was similar among patients with or without MI. The active comparator was prasugrel in one-third of the patients with $\mathrm{Ml}$ and clopidogrel in two-thirds of the patients.

In patients with MI, extended DAPT as compared with aspirin alone reduced stent thrombosis significantly $(0.5 \%$ vs. $1.9 \%$; $P<$ 0.001). There also was a significant reduction of MACCE by extended DAPT (3.9\% vs. $6.8 \%$; $P<0.001$ ). This included a major reduction in the rate of recurrent $\mathrm{MI}(2.2 \%$ vs. $5.2 \%$; $P<0.001)$. On the other side, GUSTO moderate or severe bleeding was significantly increased by extended DAPT (1.9\% vs. $0.8 \%, P=0.005)$. Contrary to the main study, all-cause mortality was similar in the extended DAPT group as compared with the placebo group ( $1.4 \%$ vs. $1.6 \% ; P=0.61)$, even if formal interaction testing was inconclusive.

A meta-analysis on the effect of extended DAPT in patients with previous $\mathrm{MI}$ comprising PEGASUS and MI subgroups of studies with thienopyridines-CHARISMA, PRODIGY, and DESLATE with clopidogrel as well as ARCTIC-Interruption and DAPT with clopidogrel or prasugrel-has been recently published. ${ }^{141}$ Extended DAPT decreased the risk of MACCE compared with aspirin alone (6.4\% vs. $7.5 \%$; $P=0.001)$. There was a consistent significant reduction in each component of the primary endpoint (RR $0.85,95 \% \mathrm{Cl} 0.74-0.98$ for cardiovascular death; RR 0.70, $95 \% \mathrm{Cl}$ $0.55-0.88$ for MI; RR $0.81,95 \% \mathrm{Cl} 0.68-0.97$ for stroke). This benefit was achieved at the cost of a significantly increased risk of major bleeding $(1.85 \%$ vs. $1.09 \%$; $P=0.004)$. Although the reduction in cardiovascular mortality associated with prolonged DAPT was significant, the absolute risk reduction was small $(0.3 \%)$. In addition, there was no difference in all-cause mortality $(4.0 \%$ in the extended DAPT group and $4.2 \%$ in the aspirin alone group). No significant difference between study heterogeneity was identified across the appraised endpoints. This may suggest a consistent class effect among the three $\mathrm{P}_{2} \mathrm{Y}_{12}$ inhibitors (clopidogrel, ticagrelor, or prasugrel). However, caution should be used in interpreting this finding, taking into account that the PEGASUS study alone contributed $\geq 60 \%$ to pooled endpoint estimates and that PEGASUS was the only trial included in its totality (and as such the only properly powered study for post-MI patients), whereas post hoc subgroups of patients recruited in the other four investigations were pooled. In addition, when the overall included populations of the four available studies assessing DAPT for $>1$ year vs. 12 month therapy are pooled, an extended treatment with ticagrelor, as compared to a similar strategy with thienopyridines, exerted a more favourable effect on all-cause mortality due to a trend towards reduction of cardiovascular death and a null effect on non-cardiovascular death. ${ }^{142}$ Finally, PEGASUS was the only trial that allowed patients who had stopped DAPT months or years before to randomly restart therapy; this likely resulted in relatively lower efficacy endpoint estimates as compared to other studies testing duration of thienopyridines where treatment was either permanently stopped or continued without treatment interruptions in between. Therefore, it is reasonable to favour ticagrelor $60 \mathrm{mg}$ b.i.d. as the agent of choice for prolonging DAPT beyond 12 months in stabilized post-MI patients at low bleeding risk, and to reserve the use of clopidogrel (or prasugrel, the least investigated agent in this setting) as the alternative choice if ticagrelor therapy is not tolerated or feasible. 
Shortening of DAPT duration in patients at high bleeding risk: There is no dedicated RCT assessing the optimal DAPT duration in patients at high bleeding risk. Moreover, many, if not all, available DAPT studies formally excluded these patients from inclusion. The Zotarolimuseluting Endeavor sprint stent in Uncertain DES Candidates (ZEUS) and the Prospective randomized comparison of the BioFreedom biolimus $A 9$ drug-coated stent versus the gazelle BMS in patients at high bleeding risk (LEADERS-FREE) studies recruited a selected high bleeding risk population and randomized them to BMS or drugcoated stent under a protocol-mandated DAPT duration of 1 month. ${ }^{129,130}$ Both studies, as discussed in section 2.4, proved the superiority of the investigated DES technologies as compared to BMS despite a similarly short duration of DAPT. The trade-off between bleeding prevention and ischaemic protection of prolonging DAPT beyond 1 month in this patient subset remains unclear.

As discussed in section 4.1, two studies compared 3- vs. 12-month DAPT duration after DES. Patients were not selected based on high bleeding risk criteria and both studies included only a minority of patients presenting with acute $\mathrm{MI}$ (14.3 and 5.4\% in the RESET and OPTIMIZE trials, respectively). ${ }^{105,106}$ associated with an estimated increase in the risk of $\mathrm{Ml}$ or definite/probable stent thrombosis from $1.7 \%$ to $2.4 \%$ compared with 1-year DAPT. Although this increase did not reach statistical significance $(\mathrm{HR} 1.48,95 \% \mathrm{Cl} 0.98-2.22 ; P=0.059)$, it has to be kept in mind that the power of this analysis was limited since the number of patients with ACS included was roughly only one-third or one-fourth of that in TRITON or PLATO, which established the superiority of intensified antiplatelet therapy over conventional 1-year DAPT with clopidogrel. Despite this limitation, it is probably fair to conclude that the ischaemic risk of shortening DAPT to 6 months after PCl in ACS is low, although not negligible. In this respect, it is also reassuring that there was no signal with respect to cardiac or all-cause death ( $\mathrm{HR} 0.75,95 \% \mathrm{Cl} 0.45-1.27$ and $\mathrm{HR} 0.85,95 \% \mathrm{Cl} 0.58-1.26$, respectively). Only when DAPT duration was reduced to 3 months did the risk of $\mathrm{Ml}$ and definite/probable stent thrombosis increase substantially $(\mathrm{HR} \quad 2.08,95 \% \mathrm{Cl} 1.10-3.93)$. In summary, currently available evidence suggests considering discontinuation of $\mathrm{P}_{2} \mathrm{Y}_{12}$ inhibitor therapy after 6 months, when the risk of bleeding is high.

\section{Dual antiplatelet therapy duration in patients with acute coronary syndrome treated with percutaneous coronary intervention}

\begin{tabular}{|c|c|c|}
\hline Recommendations & Class $^{\mathrm{a}}$ & Level $^{\mathrm{b}}$ \\
\hline $\begin{array}{l}\text { In patients with ACS treated with coronary stent implantation, DAPT with a P2Y } 12 \text { inhibitor on top of aspirin is rec- } \\
\text { ommended for } 12 \text { months unless there are contraindications such as excessive risk of bleeding (e.g. PRECISE-DAPT } \\
\geq 25 \text { ). }{ }^{20,23,40}\end{array}$ & I & $\mathbf{A}$ \\
\hline $\begin{array}{l}\text { In patients with ACS and stent implantation who are at high risk of bleeding (e.g. PRECISE-DAPT } \geq 25 \text { ), discontinua- } \\
\text { tion of } \mathrm{P}_{2} \mathrm{Y}_{12} \text { inhibitor therapy after } 6 \text { months should be considered. }{ }^{13,18,143}\end{array}$ & Ila & B \\
\hline $\begin{array}{l}\text { In patients with ACS treated with bioresorbable vascular scaffolds, DAPT for at least } 12 \text { months should be } \\
\text { considered. }\end{array}$ & Ila & C \\
\hline $\begin{array}{l}\text { In patients with ACS who have tolerated DAPT without a bleeding complication, continuation of DAPT for longer } \\
\text { than } 12 \text { months may be considered. }{ }^{26,139}\end{array}$ & Ilb & $\mathbf{A}$ \\
\hline $\begin{array}{l}\text { In patients with } \mathrm{MI} \text { and high ischaemic risk }{ }^{c} \text { who have tolerated DAPT without a bleeding complication, ticagrelor } \\
60 \mathrm{mg} \text { b.i.d. for longer than } 12 \text { months on top of aspirin may be preferred over clopidogrel or prasugrel. } .^{29,115,142}\end{array}$ & IIb & B \\
\hline
\end{tabular}

ACS = acute coronary syndrome; b.i.d. $=$ bis in die; DAPT = dual antiplatelet therapy. $\mathrm{MI}=$ myocardial infarction; PRECISE-DAPT = PREdicting bleeding Complications In patients undergoing Stent implantation and subsEquent Dual Anti Platelet Therapy.

${ }^{a}$ Class of recommendation.

'Level of evidence.

'Defined as $\geq 50$ years of age, and one or more of the following additional high-risk features: age of 65 years or older, diabetes mellitus requiring medication, a second prior spontaneous myocardial infarction, multivessel coronary artery disease, or chronic renal dysfunction, defined as an estimated creatinine clearance $<60 \mathrm{~mL} / \mathrm{min}$.

These recommendations refer to stents that are supported by large-scale randomized trials with clinical endpoint evaluation leading to unconditional CE mark, as detailed in Byrne et al. ${ }^{134}$

After an ACS, high bleeding risk status poses even greater challenges with respect to the choice of DAPT duration. The risks of shortening DAPT below 1 year have been addressed by an individual patient data meta-analysis. ${ }^{143}$ This meta-analysis comprised six trials comparing three- or six-month DAPT with 12-month DAPT including 11473 patients, 4758 of whom had ACS. In patients with ACS, shortening DAPT to $\leq 6$ months was

\subsection{Gaps in the evidence}

With a marginal overall benefit-to-risk ratio of extended DAPT beyond 1 year after DES placement, tools to identify ideal candidates for longterm or even indefinite DAPT duration are critically needed. The DAPT score $^{15}$ as well as the subgroup analyses of PEGASUS ${ }^{139,140,144,145}$ are important steps forward, but prospective validation in contemporary cohorts of newer-generation DES patients is needed. 
The optimal level of platelet inhibition during the various stages of CAD remains an open question. The risk of ischaemic complication is highest immediately after $\mathrm{PCl}$ and then gradually declines. The same is true for patients managed for ACS, although the risk remains elevated above that of patients who never experienced an acute exacerbation for years. Thus, it is intuitive that during the chronic phase after stabilization the level of platelet inhibition may be reduced as compared with the acute phase. Until recently, there were only limited data addressing this issue from beyond the periprocedural phase to 1 year. By now, two studies addressing such a step-down concept have finished recruitment: Testing Responsiveness to Platelet Inhibition on Chronic Antiplatelet Treatment For Acute Coronary Syndromes Trial (TROPICAL-ACS) (NCT01959451) with a stepdown from prasugrel to clopidogrel after the peri-interventional phase in acute Ml; and GLOBAL-LEADERS (NCT01813435) ${ }^{146}$ with a step-down from DAPT to single antiplatelet therapy with ticagrelor beyond the first month after $\mathrm{PCl}$ in an all-comers cohort with $\mathrm{DES}$.

The risks and benefits of shortening DAPT to 3 months or even shorter is another area with limited evidence. There are only two randomized studies with a total of 5236 patients. ${ }^{105,106}$ Both studies used the first-generation ZES that, due to its limited efficacy in suppressing neointima formation, has been largely replaced by a newer generation. Thus, in most cases with high bleeding risk, the decision to shorten DAPT below 6 months needs to rely on circumstantial evidence suggesting comparable safety of different stent types.

As outlined in section 4.1, there are no dedicated studies on the optimal duration of DAPT after the application of drug-eluting balloons or after implantation of a bioresorbable scaffold. It is also unclear whether, early after placement of a bioresorbable stent, patients may benefit from the more potent $\mathrm{P}_{2} \mathrm{Y}_{12}$ inhibition achieved by prasugrel or ticagrelor as compared with the current practice of clopidogrel administration.

\section{Dual antiplatelet therapy and cardiac surgery}

\subsection{Dual antiplatelet therapy in patients treated with coronary artery bypass surgery for stable coronary artery disease}

DAPT in ACS patients significantly reduces the risk of thrombotic complications but increases the risk for both spontaneous and surgical bleeding complications. ${ }^{20,23,40}$ The bleeding risk as well as the ischaemic benefit are further increased if ticagrelor or prasugrel are used instead of clopidogrel..$^{20,23}$ Unlike for ACS, there is currently no evidence of a survival benefit or a reduction of thromboembolic complications with DAPT in patients with stable CAD undergoing CABG. However, there is limited evidence suggesting that the use of DAPT in patients with stable CAD mitigates the risk of vein (but not arterial) graft occlusions.

\subsection{Dual antiplatelet therapy in patients treated with coronary artery bypass surgery for acute coronary syndrome}

Background: DAPT, as compared to aspirin monotherapy, has been proven to be beneficial in reducing ischaemic risk in ACS patients (Figure 5).

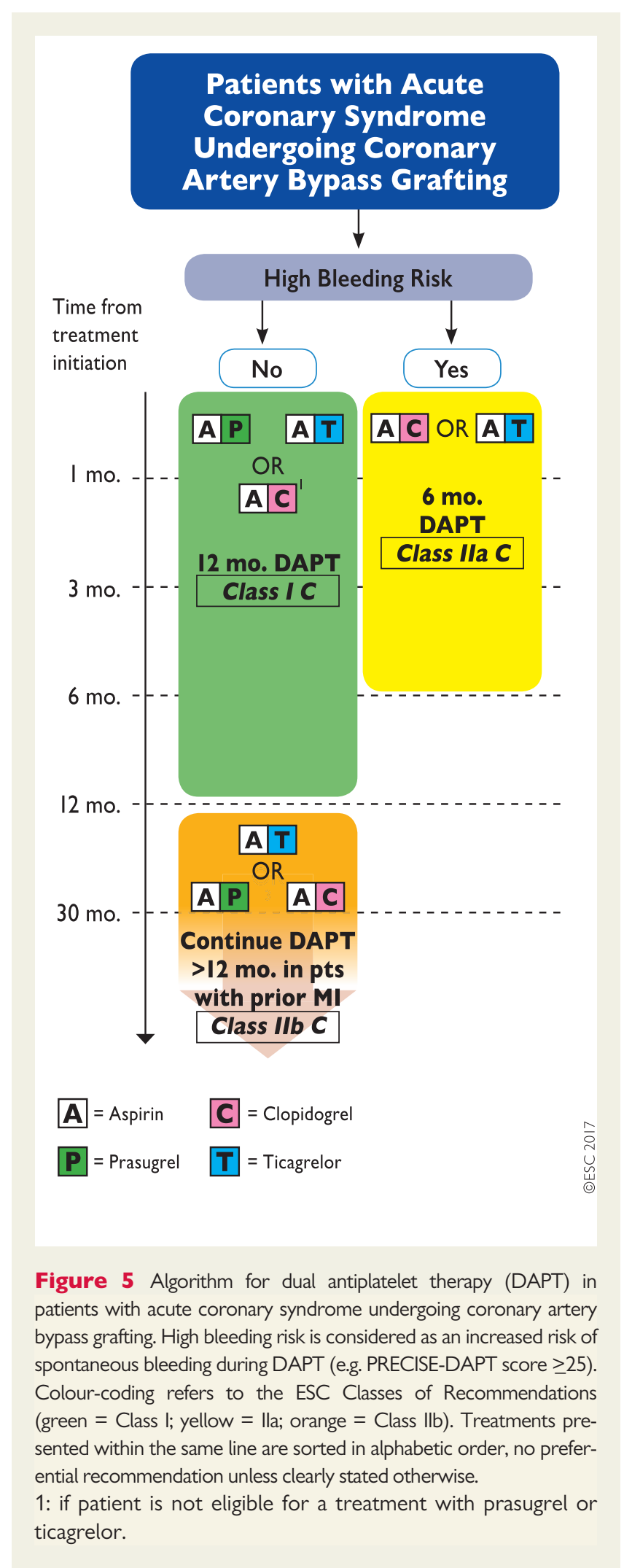

However, there is limited evidence in patients undergoing CABG as no dedicated study exists. In the Clopidogrel in Unstable Angina to Prevent Recurrent Events (CURE) trial, the outcome in the CABG 
subpopulation was consistent with the overall results of the study. ${ }^{147}$ Further support has been presented in two meta-analyses. ${ }^{148,149}$ In the CABG substudies of the TRITON-TIMI 38 and the PLATO trials where, respectively, prasugrel and ticagrelor were tested against clopidogrel in combination with ASA, both newer $\mathrm{P}_{2} \mathrm{Y}_{12}$ inhibitors were more effective than clopidogrel in preventing fatal outcomes, with a higher risk for bleeding in the former but not the latter trial. ${ }^{150,151}$

Continuation of DAPT until CABG increases the risk of excessive perioperative bleeding, transfusions, and re-exploration for bleeding as shown in RCTs, ${ }^{147,150,151}$ observational studies, ${ }^{152,153}$ and metaanalyses. ${ }^{154,155}$ Therefore, it is recommended that the $\mathrm{P} 2 \mathrm{Y}_{12}$ inhibitor be discontinued whenever possible before elective CABG. ${ }^{156,157}$ Alternatively, elective operations may be postponed until the DAPT treatment period is completed. In urgent cases, most often patients with ACS, the risk of thrombotic episodes (stent thrombosis or MI) while waiting for the effect of the $\mathrm{P}_{2} \mathrm{Y}_{12}$ inhibitor to cease must be weighed against the risk of perioperative bleeding complications. In extreme high-risk patients, e.g. those with recent DES implantation, bridging therapy with cangrelor or a glycoprotein IIb/Illa blocker may be considered. ${ }^{156,157}$

$P 2 Y_{12}$ inhibitors: The safe discontinuation interval varies between the different $P 2 Y_{12}$ inhibitors due to variations in platelet inhibitory effect and pharmacodynamic and pharmacokinetic properties. ${ }^{158}$ For clopidogrel, it was shown in the CABG substudy of the CURE trial that discontinuation $\geq 5$ days before $C A B G$ did not increase the risk of bleeding complications. ${ }^{147}$ For prasugrel, a longer time interval (7 days) is recommended due to the longer offset time compared to clopidogrel ${ }^{158}$ and the high incidence of CABG-related bleeding complications reported in the CABG substudy of the TRITON-TIMI 38 trial. $^{151}$ In CABG patients treated pre-operatively with ticagrelor, 5 days of discontinuation was initially recommended. This recommendation was based on pharmacokinetic studies and clinical data from patients with stable CAD. ${ }^{159}$ However, recent data from large observational studies in CABG patients challenge this recommendation. ${ }^{152,153,160}$ In a Swedish nationwide study, CABG-related bleeding complications in patients treated with ticagrelor or clopidogrel were thoroughly investigated with respect to timing of $\mathrm{P}_{2} \mathrm{Y}_{12}$ inhibitor discontinuation. ${ }^{152}$ When either drug was discontinued according to the instructions for use ( $>120 \mathrm{~h}$ before surgery), there was no significant difference in the incidence of major bleeding complications between ticagrelor- and clopidogrel-treated patients ( $9 \%$ vs. $12 \%$; unadjusted OR $0.72,95 \% \mathrm{Cl} 0.51-1.02 ; P=0.065)$. Within the ticagrelor group, there was no significant difference in major bleeding complications between discontinuation $72-120 \mathrm{~h}$ or $>120 \mathrm{~h}$ before surgery (OR $0.93,95 \% \mathrm{Cl}$ $0.53-1.64 ; P=0.80$ ), whereas discontinuation $0-72 \mathrm{~h}$ before surgery was associated with a significantly higher rate of major bleeding compared with both 72-120h (OR 5.17, 95\% Cl 2.89-9.27; $P<0.0001$ ) and $>120 \mathrm{~h}$ (OR 4.81, 95\% Cl 3.34-6.95; $P<0.0001$ ). In contrast, clopidogrel-treated patients had a higher incidence of major bleeding complications when discontinued $72-120 \mathrm{~h}$ compared with $>120 \mathrm{~h}$ before surgery (OR 1.71, 95\% Cl 1.04-2.79; $P=0.033)$. Likewise, in the clopidogrel group, discontinuation $0-72 \mathrm{~h}$ before surgery was associated with an increased incidence of major bleeding compared with 72-120h (OR 1.67, 95\% Cl 1.02-2.73; $P=0.042$ ) and >120h (OR 2.85, 95\% Cl 1.98-4.10; $P<0.0001$ ) (Web Figure 2, see Web Addenda). ${ }^{152}$ Further support for using 3 days as the discontinuation period in ticagrelor-treated patients comes from the PLATO trial, where a discontinuation period of 24 - $72 \mathrm{~h}$ was recommended. In a single institution Dutch registry encompassing 705 consecutive patients who underwent isolated on-pump CABG, ticagrelor discontinuation $>72 \mathrm{~h}$ and clopidogrel discontinuation $>120 \mathrm{~h}$ before surgery were not associated with an increased risk of bleeding-related complications. ${ }^{153}$

Further evidence comes from a prospective, multicentre clinical trial performed at 15 European centres, where discontinuation of ticagrelor $>2$ days before surgery was not associated with increased bleeding. ${ }^{160}$

It is unlikely that the optimal discontinuation period for any of the $\mathrm{P}_{2} \mathrm{Y}_{12}$ inhibitors will ever be tested in an RCT. As mentioned above, current guidelines recommend DAPT in all patients with ACS, independent of revascularization strategy. ${ }^{34,161}$ This applies to patients undergoing CABG and other cardiac surgical procedures as well. Furthermore, the effect of DAPT or single antiplatelet therapy after CABG has been compared in two meta-analyses based on RCTs ${ }^{148}$ or a combination of RCTs and observational studies. ${ }^{149}$ In the meta-analysis based on RCTs only (which included 3717 ACS patients), ${ }^{148}$ there were no differences in allcause mortality in ASA + clopidogrel vs. ASA only. Conversely, all-cause mortality was significantly lower in ASA + ticagrelor and ASA + prasugrel vs. ASA + clopidogrel RCTs (RR 0.49, $95 \% \mathrm{Cl}$ $0.33-0.71 ; P=0.0002$ ). There were no significant differences in occurrence of Mls, strokes, composite outcomes, or major bleeding ( $R R$ 1.31, $95 \% \mathrm{Cl} 0.81-2.10, P=0.27$ ). The meta-analysis based on both RCTs and observational studies ${ }^{149}$ included only DAPT patients treated with clopidogrel. In this analysis, inhospital or 30-day mortality was lower with ASA + clopidogrel compared to ASA alone (RR 0.38, 95\% Cl 0.26-0.57; $P<0.001$ ), while the risk of angina or perioperative $\mathrm{Ml}$ was comparable (RR 0.60, 95\% Cl 0.31-1.14; $P=0.12$ ). Long-term mortality was not reported. Patients treated with ASA + clopidogrel demonstrated a trend towards a higher incidence of major bleeding episodes as compared to patients treated with ASA alone (RR 1.17, 95\% Cl 1.00-1.37; $P=0.05)$. In both meta-analyses, there was large heterogeneity between the included studies regarding study drug (clopidogrel/prasugrel/ticagrelor), study design, patient inclusion (ACS vs. stable CAD, on-pump vs. off-pump surgery), study quality, and duration of followup. The positive effect on survival appears to be more pronounced in ACS patients and in patients treated with the second-generation $\mathrm{P}_{2} \mathrm{Y}_{12}$ inhibitors ticagrelor and prasugrel. However, re-institution of DAPT after CABG may also slightly increase the risk of bleeding complications. Thus, it is recommended that DAPT is re-started as soon as it is considered safe after CABG in ACS patients, with the exception of those on anticoagulation. There is currently no scientific support for triple antithrombotic treatment after CABG. Resuming DAPT early after CABG is most likely of special importance in patients with recent stent implantation, although strong evidence is lacking. The optimal timing of resuming DAPT remains unclear, but 24 - $96 \mathrm{~h}$ after the operation in patients without recent stent implantation appears reasonable. One reason for not starting DAPT immediately after the operation is the considerable risk ( $30 \%)$ of atrial fibrillation (AF) during the first post-operative days, which requires oral anticoagulation. ${ }^{162}$

Acetylsalicylic acid: A recent meta-analysis comparing pre-operative ASA administration vs. no treatment or placebo in CABG patients included 13 trials with a total of 2399 patients. ${ }^{163}$ The meta-analysis showed that treatment with ASA reduced the risk of perioperative 
$\mathrm{MI}(\mathrm{OR} 0.56,95 \% \mathrm{Cl} 0.33-0.96)$ but not the mortality risk (OR 1.16, $95 \% \mathrm{Cl}$ 0.42-3.22), while post-operative bleeding, red blood cell transfusions, and surgical re-explorations increased with ASA. The authors pointed out that included studies had low methodological quality. The recent Aspirin and Tranexamic Acid for Coronary Artery Surgery (ATACAS) trial compared administration of ASA $(100 \mathrm{mg})$ on the day of surgery vs. placebo in CABG patients. ${ }^{164}$ The study showed no significant effect of ASA treatment on perioperative bleeding. On the other hand, ASA treatment did not reduce the incidence of thrombotic events. It should be pointed out that the study did not directly compare discontinuation vs. no discontinuation, since the included patients were only eligible for the trial if they were not using ASA pre-operatively or had stopped ASA at least 4 days before surgery. Thus, the ATACAS study does not directly apply to the ACS-CABG population and does not change current recommendations of maintaining ASA treatment during the perioperative period.

In a case-control study on 8641 CABG patients, those pre-treated with ASA were less likely to experience in-hospital mortality in univariate (OR 0.73, 95\% Cl 0.54-0.97) and multivariate (OR 0.55, 95\% $\mathrm{Cl} 0.31-0.98)$ analysis relative to those not exposed to ASA. ${ }^{165} \mathrm{No}$ significant difference was seen in the amount of chest tube drainage, transfusion of blood products, or need for re-exploration for bleeding, between patients who were or were not exposed to ASA preoperatively.
Taken together, the evidence indicates that continuation of ASA until cardiac surgery is associated with a moderately increased risk of bleeding complications and a significant reduction in the risk of perioperative MI. If bleeding occurs during surgery, platelet transfusion has been shown to effectively counteract ASA effects. ${ }^{166-168}$ This finding further supports the possibility of continuing ASA throughout the perisurgical period as ASA allows direct antiplatelet effect reversal if clinically indicated. The increased risk of bleeding complications if ASA and other antithrombotic drugs are not discontinued should be weighed against the potentially increased risk of thrombotic complications during the pre-operative cessation period.

Platelet function testing: Besides the variance in platelet inhibitory effects between different $\mathrm{P}_{2} \mathrm{Y}_{12}$ inhibitors, there is also a large individual variation in the magnitude and duration of the antiplatelet effect. ${ }^{20,159,169-171}$ Because of the individual variation, the use of platelet function tests may aid the optimization of the timing of surgical procedures. However, platelet function tests could also be of value to establish the grade of platelet inhibition in patients in whom the time since discontinuation is unclear, e.g. in unconscious or confused patients, and in patients with uncertain compliance to the treatment.

Treatment monitoring, using bedside tests, has been suggested as an option for guiding interruption of treatment, rather than the use of an arbitrary, specified period of time. ${ }^{156,157}$ Pre-operative ADPdependent platelet aggregation capacity predicts CABG-related

\section{Dual antiplatelet therapy in patients treated with cardiac surgery with stable or unstable coronary artery disease}

\begin{tabular}{|c|c|c|}
\hline Recommendations & Class $^{a}$ & Level $^{\mathrm{b}}$ \\
\hline $\begin{array}{l}\text { It is recommended that the heart team estimates the individual bleeding and ischaemic risks, and guides the timing of } \\
C A B G \text { as well as the antithrombotic management. }\end{array}$ & I & C \\
\hline $\begin{array}{l}\text { In patients on aspirin who need to undergo non-emergent cardiac surgery, it is recommended to continue aspirin at a low } \\
\text { daily regimen throughout the perioperative period. }\end{array}$ & $\mathbf{I}$ & C \\
\hline $\begin{array}{l}\text { In patients treated with DAPT after coronary stent implantation who subsequently undergo cardiac surgery, it is recom- } \\
\text { mended to resume } \mathrm{P}_{2} \mathrm{Y}_{12} \text { inhibitor therapy post-operatively as soon as is deemed safe so that DAPT continues until the } \\
\text { recommended duration of therapy is completed. }\end{array}$ & $\mathbf{I}$ & C \\
\hline $\begin{array}{l}\text { In patients with ACS (NSTE-ACS or STEMI) treated with DAPT, undergoing CABG, and not requiring long-term OAC ther- } \\
\text { apy, resumption of } \mathrm{P} \mathrm{Y}_{12} \text { inhibitor therapy as soon as is deemed safe after surgery and continuation up to } 12 \text { months is } \\
\text { recommended. }\end{array}$ & I & C \\
\hline $\begin{array}{l}\text { In patients on } \mathrm{P}_{2} \mathrm{Y}_{12} \text { inhibitors who need to undergo non-emergent cardiac surgery, postponing surgery for at least } 3 \\
\text { days after discontinuation of ticagrelor, at least } 5 \text { days after clopidogrel, and at least } 7 \text { days after prasugrel should be } \\
\text { considered. }\end{array}$ & Ila & B \\
\hline $\begin{array}{l}\text { In CABG patients with prior MI who are at high risk of severe bleeding (e.g. PRECISE-DAPT } \geq 25 \text { ), discontinuation of P2Y } 12 \\
\text { inhibitor therapy after } 6 \text { months should be considered. }\end{array}$ & Ila & C \\
\hline $\begin{array}{l}\text { Platelet function testing may be considered to guide decisions on timing of cardiac surgery in patients who have recently } \\
\text { received } \mathrm{P}_{2} \mathrm{Y}_{12} \text { inhibitors. }{ }^{169,172-174}\end{array}$ & Ilb & B \\
\hline $\begin{array}{l}\text { In patients perceived to be at high ischaemic risk with prior MI and CABG, who have tolerated DAPT without a bleed- } \\
\text { ing complication, treatment with DAPT for longer than } 12 \text { and up to } 36 \text { months may be considered. }\end{array}$ & IIb & C \\
\hline
\end{tabular}

ACS = acute coronary syndrome; CABG = coronary artery bypass graft surgery; DAPT = dual antiplatelet therapy; MI = myocardial infarction; NSTE-ACS = non-ST-elevation acute coronary syndrome; $\mathrm{OAC}=$ oral anticoagulant; PRECISE-DAPT = PREdicting bleeding Complications in patients undergoing Stent implantation and subsEquent Dual Anti Platelet Therapy; STEMI = ST-elevation myocardial infarction.

${ }^{a}$ Class of recommendation.

bevel of evidence. 
bleeding complications in clopidogrel- ${ }^{172-174}$ and ticagrelortreated $^{169}$ ACS patients, and a strategy based on pre-operative platelet function testing, to determine the timing of CABG in clopidogreltreated patients, led to a $50 \%$ shorter waiting time than that suggested by a simple discontinuation time-based strategy. ${ }^{175}$ It should be pointed out that the different platelet function tests and their respective cut-off levels are not interchangeable. ${ }^{176}$ Taken together, these results suggest that platelet function testing in ACS patients referred for $C A B G$ is of potential value to guide the timing of surgery in patients treated with $\mathrm{P}_{2} \mathrm{Y}_{12}$ inhibitors. However, randomized studies with clinically relevant endpoints are lacking.

\subsection{Dual antiplatelet therapy for prevention of graft occlusion}

Two meta-analyses have compared graft patency in patients treated with ASA alone or ASA + clopidogrel after CABG. ${ }^{149,177}$ The studies included in the meta-analyses comprised mainly patients with stable CAD. In a meta-analysis by Deo et al, ${ }^{149}$ ASA + clopidogrel was associated with a significant reduction in saphenous vein graft occlusions (RR $0.59,95 \% \mathrm{Cl} 0.43-0.82 ; P=0.02$ ). In the meta-analysis by Nocerino et al, ${ }^{177}$ DAPT was consistently associated with a reduced occlusion rate (RR $0.63,95 \% \mathrm{Cl} 0.46-0.86)$. DAPT proved useful in preventing vein graft occlusion (RR $0.58,95 \% \mathrm{Cl} 0.42-0.83$ ), while no clear effect was shown in arterial grafts (RR $0.85,95 \% \mathrm{Cl}$ 0.39-1.85). ${ }^{177}$ Weak evidence indicates that DAPT may prevent graft occlusion in patients undergoing off-pump CABG rather than onpump CABG. ${ }^{178}$ Given the low risk of thrombotic events after $C A B G$ in stable patients, there is insufficient evidence to generally recommend DAPT post-operatively to reduce vein graft occlusion in this surgical patient subset.

\subsection{Gaps in the evidence}

There are several gaps in the evidence that pertain to the use of DAPT in cardiac surgery. Clear gaps in evidence related to DAPT in cardiac surgery patients include the question of whether DAPT should be started after CABG in patients with stable CAD. Also, the exact timing of post-operative DAPT restart remains unclear, and it remains uncertain for how long the post-operative DAPT should last. Further gaps in the evidence relate to: the optimal time point for discontinuation of the different $\mathrm{P}_{2} \mathrm{Y}_{12}$ inhibitors; the optimal use of platelet function testing in patients awaiting cardiac surgery; how to manage perioperative bleeding complications in cardiac surgery patients caused by DAPT; and whether and how an incomplete response or inadequate antiplatelet effect of aspirin after CABG should be addressed.

\section{Dual antiplatelet therapy for patients with medically managed acute coronary syndrome}

The evidence for the use of DAPT in medically managed ACS patients comes from the CHARISMA and CURE for clopidogrel, ${ }^{40,95}$ TRILOGY for prasugrel, ${ }^{24}$ and PLATO and PEGASUS for ticagrelor studies. ${ }^{20,29}$ There is no evidence in favour of prasugrel treatment in patients with ACS who are medically managed, based on the negative results of the TRILOGY study and the exclusion of this patient subset in the TRITON study. ${ }^{23,24}$ The CURE study showed a consistent benefit in ACS patients undergoing an average mean of 9 months DAPT in the form of aspirin and clopidogrel as compared to 1-month therapy in NSTE-ACS patients, irrespective of the final management strategy, including or not including coronary revascularization. ${ }^{40}$ The post-MI subset of patients in the CHARISMA trial derived significant benefit with an NNT for benefit in the range of 100, which came at the expense of higher major bleeding, with an NNT for harm of $90 .{ }^{135}$ While the post-MI population represents only a subset of those included in the CHARISMA study and the overall results of the trial did not show benefit of DAPT as compared to aspirin alone, it seems justifiable to give credit to this subanalysis based on the consistency of results within multiple recent studies; these studies showed that the long-term administration of an intensified antiplatelet regimen beyond 1 year of treatment reduced long-term ischaemic recurrences, even if at the cost of higher bleeding. ${ }^{29,179}$

Patients medically managed in the PLATO trial derived consistent benefit from ticagrelor $90 \mathrm{mg}$ b.i.d. as compared to clopidogrel. Overall mortality was also reduced in patients treated with ticagrelor $90 \mathrm{mg}$ b.i.d. $^{180}$

In the PEGASUS trial, 4271 patients had no prior coronary stent implantation and they derived consistent benefits and risks from ticagrelor vs. placebo on top of aspirin compared to patients with prior stenting.

Multiple sources have shown that medically managed ACS patients are less frequently treated with a DAPT regimen as compared to patients who received $\mathrm{PCl}^{181}$ Current evidence, especially for ticagrelor, does not support this practice and clinicians should refrain from tailoring the implementation and/or duration of a DAPT regimen depending on prior coronary stent implantation in the current era of newer-generation DES (Figure 6).

A special population that warrants specific consideration comprises patients with established NSTE-ACS in whom no lumen obstruction at coronary angiography is detected. No dedicated study exists assessing the benefits and risks of DAPT in this patient subset. However, a high prevalence of ruptured plaques has been observed at intravascular imaging modalities in this population, ${ }^{182}$ suggesting that the benefits of DAPT in preventing recurrent $\mathrm{MI}$ should not be withheld from these patients if the risk of bleeding does not outweigh the anticipated benefit.

The evidence in support of the DAPT treatment option in patients with STEMI conservatively managed or with prior lysis is limited to 1 month of treatment. ${ }^{31,32}$ Yet, in consideration of the fact that the majority of these patients would undergo invasive management afterwards, and evidence that DAPT may be beneficial irrespective of whether revascularization takes place, it is reasonable to prolong DAPT further in these patients depending on the bleeding risk. 


\section{Dual antiplatelet therapy duration in patients with acute coronary syndrome undergoing medical therapy} management.

\begin{tabular}{|c|c|c|}
\hline Recommendations & Class $^{\mathrm{a}}$ & Level $^{\mathrm{b}}$ \\
\hline $\begin{array}{l}\text { In patients with ACS who are managed with medical therapy alone and treated with DAPT, it is recommended to con- } \\
\text { tinue } \mathrm{P}_{2} \mathrm{Y}_{12} \text { inhibitor therapy (either ticagrelor or clopidogrel) for } 12 \text { months. }{ }^{20,40}\end{array}$ & $\mathbf{I}$ & $\mathbf{A}$ \\
\hline Ticagrelor is recommended over clopidogrel, unless the bleeding risk outweighs the potential ischaemic benefit. ${ }^{20}$ & $\mathbf{I}$ & B \\
\hline $\begin{array}{l}\text { In patients with medically managed ACS who are at high risk of bleeding (e.g. PRECISE-DAPT } \geq 25 \text { ), DAPT for at least } \\
1 \text { month should be considered. }\end{array}$ & Ila & C \\
\hline $\begin{array}{l}\text { In patients with prior } \mathrm{Ml} \text { at high ischaemic risk }{ }^{c} \text { who are managed with medical therapy alone and have tolerated DAPT } \\
\text { without a bleeding complication, treatment with DAPT in the form of ticagrelor } 60 \mathrm{mg} \text { b.i.d. on top of aspirin for longer } \\
\text { than } 12 \text { months and up to } 36 \text { months may be considered. }{ }^{139}\end{array}$ & Illb & B \\
\hline $\begin{array}{l}\text { In patients with prior Ml not treated with coronary stent implantation, who have tolerated DAPT without a bleeding complica- } \\
\text { tion and who are not eligible for treatment with ticagrelor, continuation of clopidogrel on top of aspirin for longer than } \\
12 \text { months may be considered. }\end{array}$ & Illb & C \\
\hline Prasugrel is not recommended in medically managed ACS patients. ${ }^{24}$ & III & B \\
\hline
\end{tabular}

ACS = acute coronary syndrome; b.i.d. = bis in die; $\mathrm{CrCl}=$ creatinine clearance; DAPT = dual antiplatelet therapy; $\mathrm{Ml}=$ myocardial infarction; PRECISE-DAPT = PREdicting bleeding Complications In patients undergoing Stent implantation and subsEquent Dual Anti Platelet Therapy.

${ }^{\mathrm{a}}$ Class of recommendation.

bLevel of evidence.

'Defined as $\geq 50$ years of age, and one or more of the following additional high-risk features: age of 65 years or older, diabetes mellitus requiring medication, a second prior spontaneous myocardial infarction, multivessel coronary artery disease, or chronic renal dysfunction, defined as an estimated creatinine clearance $<60 \mathrm{~mL} / \mathrm{min}$.

\section{Dual antiplatelet therapy for patients with indication for oral anticoagulation}

\subsection{Risk stratification and strategies to improve outcome after percutaneous coronary intervention}

Approximately $6-8 \%$ of patients undergoing $\mathrm{PCl}$ have an indication for long-term oral anticoagulants (OACs) due to various conditions such as $\mathrm{AF}$, mechanical heart valves, or venous thromboembolism. Compared with oral anticoagulation therapy alone, the addition of DAPT to OAC therapy results in at least a two- to threefold increase in bleeding complications. ${ }^{183-186}$ Therefore, these patients should be considered at high risk of bleeding, and the indication for OAC should be reassessed and treatment continued only if a compelling indication exists \{e.g. paroxysmal, persistent, or permanent $\mathrm{AF}$ with a $\mathrm{CHA}_{2} \mathrm{DS}_{2}$ VASc [Cardiac failure, Hypertension, Age $\geq 75$ (2 points), Diabetes, Stroke (2 points)-Vascular disease, Age 65-74, Sex category] score $\geq 1$ in men, $\geq 2$ in women; mechanical heart valve; recent (i.e. 6 months) or a history of recurrent deep venous thrombosis or pulmonary embolism\}. Conversely, every effort should be undertaken to implement strategies to minimize $\mathrm{PCl}$-related complications in these patients (Table 4). In particular, the duration of triple therapy should be limited or omitted after hospital discharge (i.e. confined to the periprocedural phase with aspirin being stopped thereafter), taking into account the ischaemic (e.g. complexity of treated CAD, amount of disease left untreated, technical considerations regarding stent implantation techniques, and results) as well as the bleeding risks. While ischaemic risk scores also predict bleeding outcomes in $A F,{ }^{187}$ suggesting considerable overlap among risk factors associated with ischaemic and bleeding outcomes, multiple bleeding risk scores, ${ }^{188}$ including the HAS-BLED ${ }^{189}$ [Hypertension, Abnormal renal and liver function (1 point each), Stroke, Bleeding history or predisposition, Labile INR, Elderly (> 65 years), Drugs and alcohol (1 point each)] score, have been shown to outperform $\mathrm{CHADS}_{2}$ [Cardiac failure, Hypertension, Age, Diabetes, Stroke (Doubled)] or $\mathrm{CHA}_{2} \mathrm{DS}_{2}$-VASc in predicting bleeding risk.

Importantly, HAS-BLED draws attention to the reversible bleeding risk factors to be addressed by the responsible clinician during the follow-up. Risk is not static and, particularly for bleeding, many risk factors can be modified. Hence, a high risk of bleeding (e.g. HAS-BLED score $\geq 3$ ) is not a reason to withhold OAC; instead, such patients should be 'flagged-up' for more careful review and follow-up.

More recently, the novel biomarker-based ABC [Age, Biomarkers (GDF-15, cTnT-hs, and haemoglobin), and Clinical history (previous bleeding) $]^{190}$ bleeding risk score has been generated and validated in a broad AF population treated with both vitamin $\mathrm{K}$ antagonist (VKA) and non-vitamin K oral anticoagulants (NOACs), and has shown superior prediction capability as compared to HAS-BLED. However, similar to all other bleeding risk scores, none of these risk prediction models developed for OAC patients has been prospectively tested in the setting of prospective RCTs. Therefore, their value in improving patient outcomes remains unclear.

A comprehensive list of all risk factors that have been associated with greater bleeding risk has been previously published. ${ }^{162}$

In the absence of safety and efficacy data from RCTs [only $6 \%$ of patients were treated at baseline with ticagrelor or prasugrel in the Rivaroxaban and a dose-adjusted oral VKA treatment strategy in 


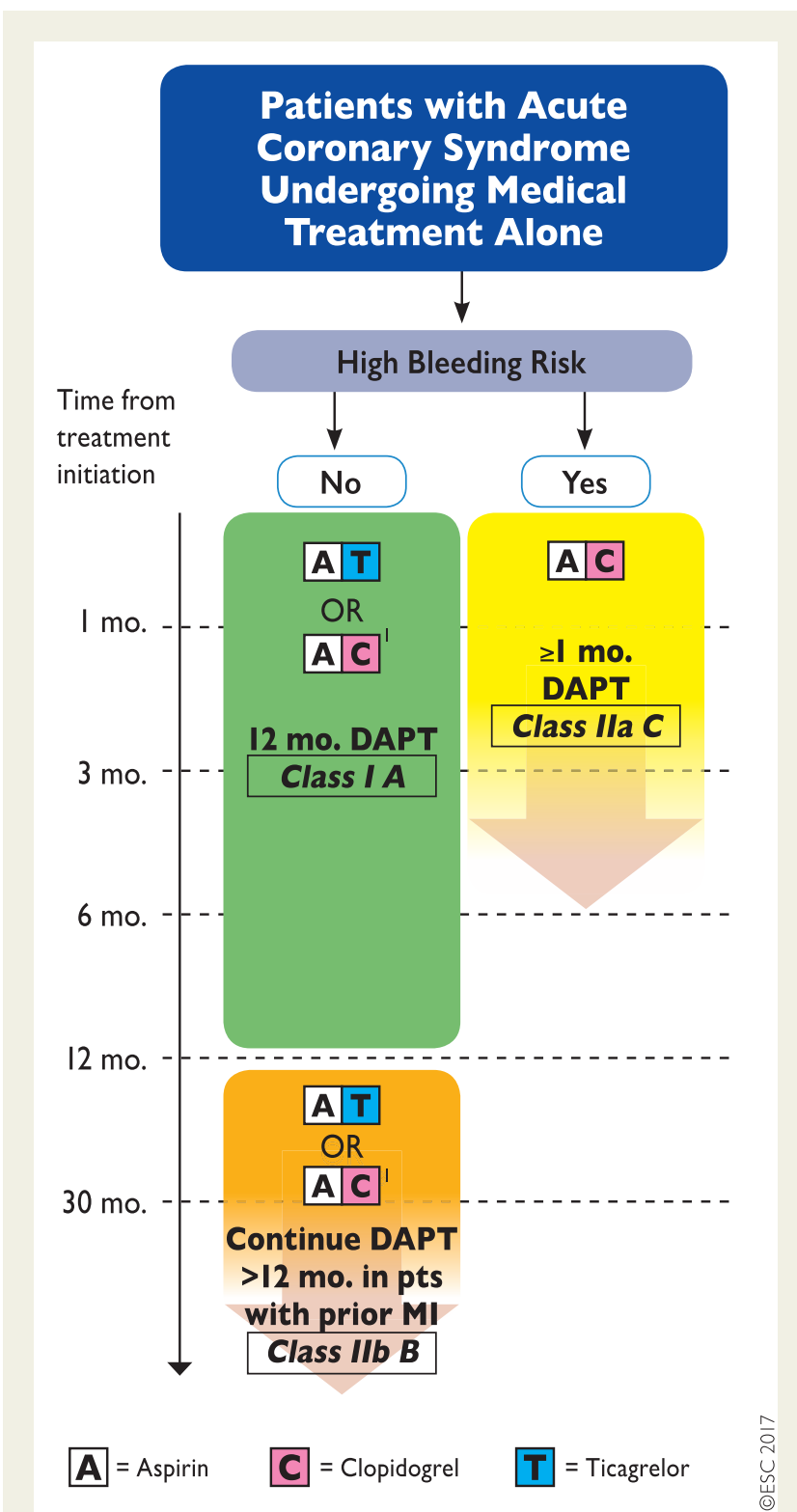

Figure 6 Algorithm for dual antiplatelet therapy (DAPT) in patients with acute coronary syndrome undergoing medical management. High bleeding risk is considered as an increased risk of spontaneous bleeding during DAPT (e.g. PRECISE-DAPT score $\geq 25$ ). Colour-coding refers to the ESC Classes of Recommendations (green $=$ Class I; yellow $=$ lla; orange $=$ Class llb). Treatments presented within the same line are sorted in alphabetic order, no preferential recommendation unless clearly stated otherwise.

1: if patient is not eligible for a treatment with ticagrelor

subjects with atrial fibrillation who undergo percutaneous coronary intervention (PIONEER AF-PCl) study ${ }^{191}$ ] and worrisome bleeding signals in registries, the use of prasugrel or ticagrelor as part of triple therapy should be avoided. ${ }^{192}$ Gastric protection with a PPI is recommended. The dose intensity of OAC should be carefully monitored with a target international normalized ratio (INR) in the lower part of the recommended target range; in patients treated with NOACs, the
Table 4 Strategies to avoid bleeding complications in patients treated with oral anticoagulant

\begin{tabular}{|l|}
\hline - Assess ischaemic and bleeding risks using validated risk predictors \\
(e.g. $\mathrm{CHA}_{2} \mathrm{DS}_{2}$-VASc, $\mathrm{ABC}, \mathrm{HAS}-\mathrm{BLED}$ ) with a focus on modifiable \\
risk factors. \\
\hline - Keep triple therapy duration as short as possible; dual therapy after \\
$\mathrm{PCl}$ (oral anticoagulant and clopidogrel) to be considered instead of \\
triple therapy. \\
\hline - Consider the use of NOACs instead of VKA. \\
\hline - Consider a target INR in the lower part of the recommended target \\
range and maximize time in therapeutic range (i.e. $>65-70 \%$ ) when \\
VKA is used. \\
\hline - Consider the lower NOAC regimen tested in approval studies and \\
apply other NOAC regimens based on drug-specific criteria for \\
drug accumulation. \\
\hline - Clopidogrel is the P2Y $\mathrm{Y}_{12}$ inhibitor of choice. \\
- Use low-dose ( $\leq 100$ mg daily) aspirin. \\
\hline - Routine use of PPIs. \\
\hline
\end{tabular}

$\mathrm{ABC}=$ Age, Biomarkers, Clinical history; $\mathrm{CHA}_{2} \mathrm{DS}_{2}-\mathrm{VASc}=$ Congestive heart failure, Hypertension, Age $\geq 75$ years (doubled), Diabetes mellitus, prior Stroke or transient ischaemic attack or thromboembolism (doubled), Vascular disease, Age 65-74 years, Sex category; HAS-BLED = Hypertension, Abnormal renal/liver function, Stroke, Bleeding history or predisposition, Labile INR, Elderly, Drugs/ alcohol concomitantly; NOAC = non-vitamin-K oral anticoagulant; INR = international normalized ratio; $\mathrm{PCl}=$ percutaneous coronary intervention; $\mathrm{PPIs}=$ proton pump inhibitors; VKA = vitamin $\mathrm{K}$ antagonist.

${ }^{\text {a Apixaban }} 5 \mathrm{mg}$ b.i.d or apixaban $2.5 \mathrm{mg}$ b.i.d. if at least two of the following: age $\geq 80$ years, body weight $\leq 60 \mathrm{~kg}$ or serum creatinine level $\geq 1.5 \mathrm{mg} / \mathrm{dL}(133 \mu \mathrm{mol} /$ L); dabigatran $110 \mathrm{mg}$ b.i.d.; edoxaban $60 \mathrm{mg}$ q.d. or edoxaban $30 \mathrm{mg}$ q.d. if any of the following: creatinine clearance $(\mathrm{CrCl})$ of $30-50 \mathrm{~mL} / \mathrm{min}$, body weight $\leq 60 \mathrm{~kg}$, concomitant use of verapamil or quinidine or dronedarone; rivaroxaban $20 \mathrm{mg}$ q.d. or rivaroxaban $15 \mathrm{mg}$ q.d. if $\mathrm{CrCl} 30-49 \mathrm{~mL} / \mathrm{min}$.

lowest effective tested dose for stroke prevention should be applied and criteria for drug accumulation for each approved NOAC should be carefully assessed. Lower NOAC regimens as compared to those tested in approval studies are expected to decrease bleeding risk, but the trade-off between bleeding and ischaemic (i.e. stroke prevention) outcomes remains largely undefined. The PIONEER AF-PCl study ${ }^{191}$ (described in detail below) tested two lower rivaroxaban doses ( $15 \mathrm{mg}$ o.d. and $2.5 \mathrm{mg}$ b.i.d.) as compared to the approved drug regimen in AF patients (20 mg q.d.). The Evaluation of Dual Therapy With Dabigatran vs. Triple Therapy With Warfarin in Patients With AF That Undergo a PCI With Stenting (REDUAL-PCl; NCT02164864) will compare two dabigatran doses $(150 \mathrm{mg}$ b.i.d. and $110 \mathrm{mg}$ b.i.d.) vs. VKA and will provide additional insights with respect to the balance between efficacy and safety for each one. Whether there are differences according to the type of OAC (NOACs vs. VKA) or stent platform as well the duration of triple therapy is further discussed. These considerations do not pertain to medically managed patients or to patients eligible for CABG surgery in whom DAPT should be avoided on top of OAC.

\subsection{Duration of triple therapy}

Cessation of aspirin after $\mathrm{PCl}$ while maintaining clopidogrel has been evaluated in the What is the Optimal antiplatElet and anticoagulant 


\section{Patients with an indication for oral anticoagulation} undergoing PCl'

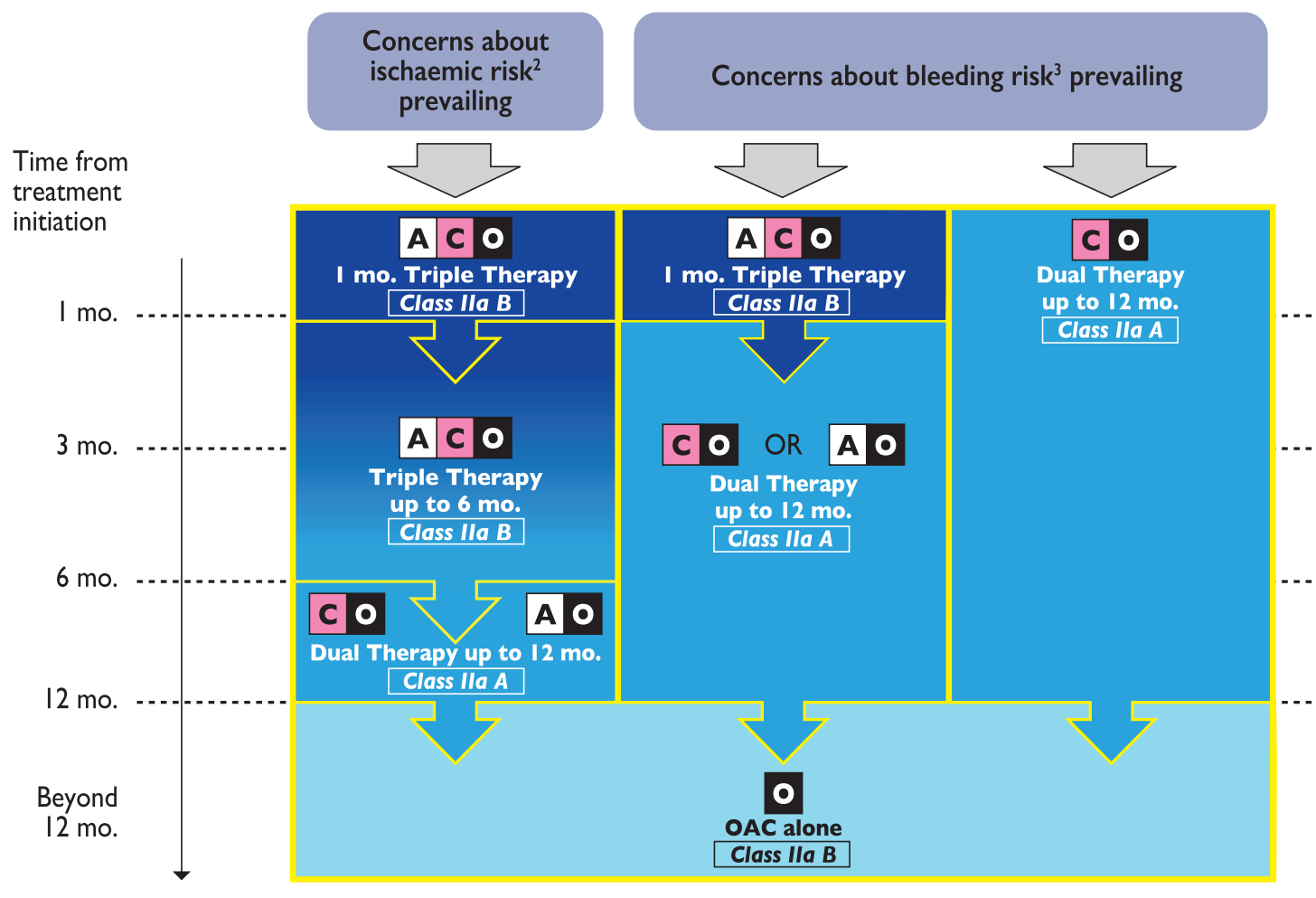

$\mathbf{A}=$ Aspirin $\quad \mathbf{C}=$ Clopidogrel $\quad \mathbf{O}=$ Oral anticoagulation

Figure 7 Algorithm for dual antiplatelet therapy (DAPT) in patients with an indication for oral anticoagulation undergoing percutaneous coronary intervention (PCl). Colour-coding refers to the number of concomitant antithrombotic medication(s). Triple therapy denotes treatment with DAPT plus oral anticoagulant (OAC). Dual therapy denotes treatment with a single antiplatelet agent (aspirin or clopidogrel) plus OAC. $\mathrm{ABC}=$ age, biomarkers, clinical history; $\mathrm{ACS}=$ acute coronary syndrome; $\mathrm{mo} .=$ month $(\mathrm{s}) ; \mathrm{PCl}=$ percutaneous coronary intervention. 1: Periprocedural administration of aspirin and clopidogrel during $\mathrm{PCl}$ is recommended irrespective of the treatment strategy.

2: High ischaemic risk is considered as an acute clinical presentation or anatomical/procedural features which might increase the risk for myocardial infarction.

3: Bleeding risk can be estimated by HAS-BLED or ABC score.

therapy in patients with OAC and coronary StenTing (WOEST) trial, which randomized 573 patients (of whom $69 \%$ of patients had AF) to dual therapy with OAC and clopidogrel $(75 \mathrm{mg} /$ day $)$ or to triple therapy with OAC, clopidogrel, and aspirin $80 \mathrm{mg} /$ day. ${ }^{193}$ Treatment was continued for 1 month after BMS placement and for 1 year after DES placement (65\% of patients). $\mathrm{PCl}$ was performed on VKA therapy in half of the patients. The primary endpoint of any TIMI bleeds assessed at 1-year follow-up was significantly reduced in the dual-therapy arm (19.5\% vs. 44.9\%; HR 0.36, 95\% Cl 0.26-0.50; $P<0.001$ ), while no significant difference in major bleeding was observed. The rates of $\mathrm{MI}$, stroke, target vessel revascularization, or stent thrombosis did not differ significantly, but all-cause mortality was lower in the dualtherapy group ( $2.5 \%$ vs. $6.4 \% ; P=0.027)$ at 1 year.
More recently, the PIONEER AF-PCI study randomized 2124 patients with non-valvular AF who had undergone $\mathrm{PCl}$ with stenting to receive, in a 1:1:1 ratio: low-dose rivaroxaban (15 mg o.d.) plus a $\mathrm{P}_{2} \mathrm{Y}_{12}$ inhibitor (and no ASA) for 12 months; very-low-dose rivaroxaban (2.5 mg b.i.d.) plus DAPT for 1, 6, or 12 months; or standard therapy with a dose-adjusted VKA plus DAPT for 1, 6, or 12 months. ${ }^{191}$ The primary safety endpoint, consisting of TIMI clinically significant bleeding, was lower in the two groups receiving rivaroxaban than in the group receiving standard therapy $[16.8 \%$ in patients treated with rivaroxaban $15 \mathrm{mg}, 18 \%$ in patients treated with rivaroxaban $2.5 \mathrm{mg}$, and $26.7 \%$ in patients treated with triple therapy $(\mathrm{HR} \mathrm{0.59,95 \%} \mathrm{Cl}$ 0.47-0.76; $P<0.001$, and HR 0.63, 95\% Cl 0.50-0.80; $P<0.001$, respectively)]. It is worth mentioning that as many as $49 \%$ of patients 
in both DAPT groups continued triple therapy for 12 months and no difference in major bleeding or transfusion was observed across the groups. Moreover, an INR range of 2-3 was recommended, instead of 2-2.5, which may have inflated bleeding risk in the control group. The rates of all-cause death, death from cardiovascular causes, MI, or stroke were similar in the three groups. ${ }^{194}$ However, this study, similar to WOEST, was largely underpowered for the assessment of meaningful differences in the incidence of relevant ischaemic events such as stent thrombosis or stroke rates. Therefore, uncertainty remains regarding the comparative performance of three tested antithrombotic regimens in patients at high stroke and/or stent thrombosis risk. Procedural characteristics of coronary intervention have not been reported so far and patients with prior stroke were excluded from participation. As a result, the balance of ischaemic and bleeding risks of relatively short (i.e. 6 months or less) triple therapy duration (possibly with NOAC instead of VKA) as compared to double therapy consisting of clopidogrel and OAC remains unknown and requires a patient-by-patient decision.

Dual therapy with clopidogrel and $\mathrm{OAC}$ after $\mathrm{PCl}$ remains an appealing alternative to triple therapy given that patients exposed to OAC are at high bleeding risk, but more data, especially on efficacy and particularly in patients at high risk for stroke and/or recurrent ACS, are needed. Cessation of clopidogrel while maintaining aspirin has also been tested in the Triple Therapy in Patients on Oral Anticoagulation After Drug Eluting Stent Implantation (ISAR-TRIPLE) trial, where 614 patients (one-third with ACS) undergoing stenting and requiring OAC were randomly assigned to receive either 6week or 6-month clopidogrel therapy in addition to aspirin and VKA. ${ }^{195}$ The primary endpoint of death, Ml, stent thrombosis, ischaemic stroke, or TIMI major bleeding at 9 months did not differ between the 6-week and 6-month triple therapy (9.8\% vs. $8.8 \%$; HR $1.14,95 \% \mathrm{Cl} 0.68-1.91$; $P=0.63)$; the same was true for the combined incidence of death, $\mathrm{Ml}$, stent thrombosis, and ischaemic stroke (4.0\% vs. 4.3\%; HR 0.93, 95\% Cl 0.43-2.05; $P=0.87$ ). Furthermore, no difference in TIMI major bleeding (5.3\% vs. $4.0 \%$; $\mathrm{HR} 1.35,95 \% \mathrm{Cl}$ $0.64-2.84 ; P=0.44$ ) was observed.

In all three studies, roughly one-third of patients presented with ACS. There was no interaction between the duration of triple therapy and clinical presentation (ACS vs. no ACS), which may reflect a real lack of increased coronary ischaemic risk in these patients or a lack of power to detect clinically meaningful differences in coronary ischaemic outcomes if these patients undergo shorter duration of DAPT regimen (i.e. 1 month ${ }^{195}$ or immediate discontinuation of aspirin after $\mathrm{PCl}^{191,193}$ ). The rate of bleeding events peaked within the first 30 days of initiation of triple therapy and was twice as high when compared with the rate of acute coronary events including recurrent $\mathrm{Ml}$ and stent thrombosis. These observations are consistent with the nationwide Danish registry of AF allcomers with $\mathrm{MI}$, where the 90 -day bleeding risk was increased on triple therapy compared with OAC plus a single antiplatelet agent (HR 1.47, 95\% Cl 1.04-2.08), with a consistent trend at 360 days (HR 1.36, 95\% $\mathrm{Cl}$ 0.95-1.95), without differences in ischaemic events (HR 1.15, 95\% $\mathrm{Cl} 0.95-1.40) .{ }^{196}$ The same registry suggests that warfarin plus clopidogrel resulted in a non-significant reduction in major bleeds (HR 0.78, 95\% Cl 0.55-1.12) compared with triple therapy, with a non-significant reduction in $\mathrm{Ml}$ or coronary death (HR 0.69, 95\% Cl 0.55-1.12). ${ }^{197}$ For these reasons, duration of triple therapy should be minimized depending on bleeding and ischaemic risks (Figure 7; Tables 5 and 6).
Dual antiplatelet therapy duration in patients with indication for oral anticoagulation

\begin{tabular}{|c|c|c|}
\hline Recommendations & Class $^{a}$ & Level $^{\mathrm{b}}$ \\
\hline $\begin{array}{l}\text { It is recommended to administer periproce- } \\
\text { durally aspirin and clopidogrel in patients } \\
\text { undergoing coronary stent implantation. }\end{array}$ & I & C \\
\hline $\begin{array}{l}\text { In patients treated with coronary stent implan- } \\
\text { tation, triple therapy with aspirin, clopidogrel, } \\
\text { and OAC should be considered for } 1 \text { month, } \\
\text { irrespective of the type of stent used. }{ }^{195}\end{array}$ & Ila & B \\
\hline $\begin{array}{l}\text { Triple therapy with aspirin, clopidogrel, and } \\
\text { OAC for longer than } 1 \text { month and up to } \\
6 \text { months should be considered in patients } \\
\text { with high ischaemic risk due to ACS or } \\
\text { other anatomical/procedural characteristics } \\
\text { that outweigh the bleeding risk. }{ }^{195}\end{array}$ & Ila & B \\
\hline $\begin{array}{l}\text { Dual therapy with clopidogrel } 75 \mathrm{mg} / \text { day } \\
\text { and OAC should be considered as an alter- } \\
\text { native to } 1 \text {-month triple antithrombotic } \\
\text { therapy in patients in whom the bleeding } \\
\text { risk outweighs the ischaemic risk. }{ }^{191,193}\end{array}$ & Ila & $\mathbf{A}$ \\
\hline $\begin{array}{l}\text { Discontinuation of antiplatelet treatment in } \\
\text { patients treated with OAC should be con- } \\
\text { sidered at } 12 \text { months. }{ }^{198}\end{array}$ & Ila & B \\
\hline $\begin{array}{l}\text { In patients with an indication for VKA in combi- } \\
\text { nation with aspirin and/or clopidogrel, the dose } \\
\text { intensity of VKA should be carefully regulated } \\
\text { with a target INR in the lower part of the rec- } \\
\text { ommended target range and a time in the ther- } \\
\text { apeutic range }>65-70 \%{ }^{193,195}\end{array}$ & Ila & B \\
\hline $\begin{array}{l}\text { When a NOAC is used in combination with } \\
\text { aspirin and/or clopidogrel, the lowest approved } \\
\text { dose effective for stroke prevention tested in } \\
\text { AF trials should be considered. }{ }^{c}\end{array}$ & Ila & C \\
\hline $\begin{array}{l}\text { When rivaroxaban is used in combination with } \\
\text { aspirin and/or clopidogrel, rivaroxaban } 15 \mathrm{mg} \text { q.d. } \\
\text { may be used instead of rivaroxaban } 20 \mathrm{mg} \text { q.d. }{ }^{191}\end{array}$ & Ilb & B \\
\hline $\begin{array}{l}\text { The use of ticagrelor or prasugrel is not rec- } \\
\text { ommended as part of triple antithrombotic } \\
\text { therapy with aspirin and OAC. }\end{array}$ & III & C \\
\hline
\end{tabular}

$\mathrm{ACS}=$ acute coronary syndrome; $\mathrm{AF}=$ atrial fibrillation; b.i.d. $=$ bis in die; $\mathrm{CrCl}=$ creatinine clearance; $I N R=$ international normalized ratio; $N O A C=$ non-vitamin $\mathrm{K}$ oral anticoagulant; $\mathrm{OAC}=$ oral anticoagulant; $q$. .d. $=$ quaque die; $\mathrm{VKA}=$ vitamin $\mathrm{K}$ antagonist.

${ }^{\mathrm{a} C}$ Class of recommendation.

bLevel of evidence.

cApixaban $5 \mathrm{mg}$ b.i.d or apixaban $2.5 \mathrm{mg}$ b.i.d. if at least two of the following: age $\geq 80$ years, body weight $\leq 60 \mathrm{~kg}$ or serum creatinine level $\geq 1.5 \mathrm{mg} / \mathrm{dL}(133 \mu \mathrm{mol} /$ L); dabigatran $110 \mathrm{mg}$ b.i.d.; edoxaban $60 \mathrm{mg}$ q.d. or edoxaban $30 \mathrm{mg}$ q.d. if any of the following: $\mathrm{CrCl}$ of $30-50 \mathrm{~mL} / \mathrm{min}$, body weight $\leq 60 \mathrm{~kg}$, concomitant use of verapamil, quinidine, or dronedarone; rivaroxaban $20 \mathrm{mg}$ q.d. or rivaroxaban $15 \mathrm{mg}$ q.d. if $\mathrm{CrCl} 30-49 \mathrm{~mL} / \mathrm{min}$. 
Table 5 High-risk features of stent-driven recurrent ischaemic events

\begin{tabular}{|l|}
\hline - Prior stent thrombosis on adequate antiplatelet therapy \\
\hline - Stenting of the last remaining patent coronary artery \\
\hline - Diffuse multivessel disease especially in diabetic patients \\
\hline - Chronic kidney disease (i.e. creatinine clearance $<60 \mathrm{~mL} / \mathrm{min}$ ) \\
\hline - At least three stents implanted \\
\hline - At least three lesions treated \\
\hline - Bifurcation with two stents implanted \\
\hline - Total stent length $>60 \mathrm{~mm}$ \\
\hline - Treatment of a chronic total occlusion \\
\hline
\end{tabular}

Table 6 Unfavourable patient profile for a combination of oral anticoagulant and antiplatelet therapy

\begin{tabular}{|l|}
\hline - Short life expectancy \\
\hline - Ongoing malignancy \\
\hline - Poor expected adherence \\
\hline - Poor mental status \\
\hline - End stage renal failure \\
\hline - Advanced age \\
\hline - Prior major bleeding/prior haemorrhagic stroke \\
\hline - Chronic alcohol abuse \\
\hline - Anaemia \\
\hline - Clinically significant bleeding on dual antithrombotic therapy \\
\hline
\end{tabular}

\subsection{Cessation of all antiplatelet agents}

Data on the timing of cessation of any antiplatelet agents in stented patients requiring chronic OAC are scarce. In stabilized event-free patients, discontinuation of any antiplatelet agent at 1 year after stenting is encouraged in this patient population based on studies demonstrating that OACs alone are superior to aspirin post-ACS, and OAC + aspirin may not be more protective but associated with excess bleeding. ${ }^{198}$ Dual therapy with OAC and one antiplatelet agent (aspirin or clopidogrel) may be considered beyond 1 year in patients at very high risk of coronary events as defined in Table $5^{34}$ and in patients with mechanical prosthesis and atherosclerotic disease.

\subsection{Type of anticoagulants}

PIONEER AF-PCl is the only randomized study comparing VKAs and NOACs in patients with $A F$ undergoing $P C I$ for ACS or for stable CAD (i.e. patients who have an indication to receive DAPT). ${ }^{191}$ However, in this study, two non-approved rivaroxaban regimens for AF patients were tested and a low (i.e. $15 \mathrm{mg}$ q.d.) or very low (i.e. $2.5 \mathrm{mg}$ b.i.d.) rivaroxaban dose in combination with a single $\mathrm{P}_{2} \mathrm{Y}_{12}$ inhibitor or DAPT was compared to VKA plus DAPT, respectively. The study was underpowered for ischaemic endpoints. Therefore, no conclusion can be made on the advantages and limitations of each OAC as compared to others. However, there was an excess of stroke events in the $2.5 \mathrm{mg}$ b.i.d. rivaroxaban arm in combination with 6-month DAPT as compared to VKA and 6-month DAPT ( 6 vs. 0 events; $P=0.02$ ).

In the four phase III NOAC AF trials, no interactions were demonstrated between treatment effect and outcome according to prior coronary status (ACS vs. no ACS), and it is likely that the benefit of NOAC over VKA is preserved in CAD patients with AF. ${ }^{199-202}$ At least, this was the case among patients exposed to antiplatelet therapy. There is no strong evidence for choosing one NOAC over another. Dabigatran is the only NOAC that has been tested in a phase III trial at reduced daily regimen (i.e. $110 \mathrm{mg}$ b.i.d.) and for which non-inferiority vs. warfarin was shown. ${ }^{199}$ Although lower doses of other NOACs (i.e. apixaban $2.5 \mathrm{mg}$ b.i.d. or edoxaban $30 \mathrm{mg}$ o.d.) might be considered to reduce bleeding risk, these dosages have been evaluated only in a subset of patients in the phase III trials based on prespecified dosing algorithms. Their benefit in stroke prevention in patients with a normal renal function is uncertain. Three ongoing large-scale outcome studies are evaluating combinations of NOACs or VKAs with antiplatelet therapy in AF patients undergoing stentPCI (NCT02164864, NCT02415400, and NCT02866175). Various dose regimens of NOAC, different types of $\mathrm{P}_{2} \mathrm{Y}_{12}$ inhibitors, and different exposure times are being evaluated.

\subsection{Type of stent}

The choice of newer-generation DES vs. BMS in patients requiring long-term anticoagulation is no longer controversial. First, data from the DAPT trial indicate a similar impact of prolonged DAPT administration irrespective of stent type (BMS vs. DES), ${ }^{128}$ and the risk of adverse events among patients with DAPT cessation and patients undergoing non-cardiac surgery indicate no differences between BMS and DES. 17,129,203 Second, two randomized trials have demonstrated the superiority of newer-generation DES over BMS in high bleeding risk patients who cannot tolerate long-term exposure to DAPT, ${ }^{130,204}$ such as those needing chronic OAC (section 2.2).

Altogether, both trials suggest that second-generation DES should be the default choice in patients with high bleeding risk.

\section{Elective non-cardiac surgery in patients on dual antiplatelet therapy}

It is estimated that $5-25 \%$ of patients with coronary stents may require non-cardiac surgery within 5 years after stent implantation. ${ }^{205}$ Management of patients on DAPT who are referred for surgical procedures involves consideration of: (1) the risk of stent thrombosis (particularly if DAPT needs to be interrupted); (2) the consequences of delaying the surgical procedure; and (3) the increased intra- and periprocedural bleeding risk and possible consequences of such bleeding if DAPT is continued. ${ }^{206-208}$ Given the complexity of these considerations, a multidisciplinary approach -involving interventional cardiologists, cardiologists, anaesthetists, haematologists, and surgeons - is required to determine the patient's risk for bleeding and thrombosis and to choose the best management strategy. 
Surgical interventions can be divided into low-risk, intermediate-risk, and high-risk groups, with estimated 30-day cardiac event rates for cardiac death or $\mathrm{Ml}$ of $<1 \%, 1-5 \%$, and $\geq 5 \%$, respectively. ${ }^{205,209}$ A practical classification of the bleeding risk associated with each type of non-cardiac surgery has been recently proposed by the Stent After Surgery group. ${ }^{210}$

In surgical procedures with low bleeding risk, every effort should be taken not to discontinue DAPT perioperatively. In surgical procedures with moderate bleeding risk, patients should be maintained on aspirin while $\mathrm{P}_{2} \mathrm{Y}_{12}$ inhibitor therapy should be discontinued whenever possible. More challenging decision making is to be faced among patients on DAPT who undergo high bleeding risk non-cardiac surgeries, including vascular reconstructions, complex visceral procedures, neurosurgery, and transbronchial operations. ${ }^{211-213}$ In these cases, particular attention should be paid to timely discontinuation of $\mathrm{P}_{2} \mathrm{Y}_{12}$ inhibitor therapy to minimize the off-therapy period before surgical intervention.

Discontinuation before non-cardiac surgery: To reduce the risk of bleeding and transfusion, it is recommended to postpone elective non-cardiac surgery until completion of the full course of DAPT. In most clinical situations, aspirin provides benefit that outweighs the bleeding risk and should be continued. ${ }^{214,215}$ Possible exceptions to this recommendation include intracranial procedures, transurethral prostatectomy, intraocular procedures, and operations with extremely high bleeding risk. ${ }^{157}$

A higher risk of ischaemic events in the case of non-cardiac surgery has been reported after first-generation $\mathrm{DES}^{203}$ and a higher risk for MACE has also been shown during the first weeks after non-cardiac surgery in patients with implanted stents. ${ }^{203,216,217}$ Furthermore, surgery per se, irrespective of the timing of DAPT discontinuation, is associated with pro-inflammatory and pro-thrombotic effects, thereby increasing the risk of coronary thrombosis at the level of the stented vascular segment as well as throughout the coronary vasculature. ${ }^{218,219}$ Therefore, in patients undergoing non-cardiac surgery after recent ACS or stent implantation, the benefits of early surgery for a specific pathology (e.g. malignant tumours or vascular aneurysm repair) should be balanced against the risk of cardiovascular events and the strategy should be discussed by a multidisciplinary team.

Prior recommendations with regard to duration of DAPT 220,221 and the timing of non-cardiac surgery ${ }^{207,222}$ in patients treated with DES were based on observations of those treated with firstgeneration DES. Compared with first-generation DES, currently used newer-generation DES are associated with a lower risk of stent thrombosis and appear to require a shorter minimum duration of DAPT. ${ }^{100,103,104,223-225}$ Furthermore, in the PARIS registry, interruption of DAPT grounded on physician judgment in patients undergoing surgery at any time point after $\mathrm{PCl}$ was not associated with an increased risk of MACE. ${ }^{17}$

In the absence of a surgical control group, it remains challenging to identify a clear time frame after ACS or coronary stenting where there is no additional risk or the risk is acceptably low for patients to undergo surgery. Therefore, almost all registries have attempted to identify such landmarks by looking at the time course of the surgical ischaemic risk over time in order to identify when it levels off and remains stable thereafter following an ACS or stent implantation procedure. ${ }^{17}$ By doing so, many registries have reported that surgeryassociated risk in DES-PCl-treated patients reaches a stable level after 3-6 months. ${ }^{17,214,215}$ However, without a surgical control group, these findings are potentially influenced by the type and urgency of the surgical procedures. To overcome this limitation, two large matched cohorts of patients undergoing surgery were recently reported. Using Danish population-based registries and individualbased record linkage of Danish registries, 4303 DES-PCl-treated patients who underwent a surgical procedure within 12 months were identified and were compared with a control group of patients without established stable CAD undergoing similar surgical procedures ( $n$ $=20232) .{ }^{226}$ This evaluation of the comparative risk associated with surgery in DES-PCl-treated patients vs. patients without known stable CAD revealed an increased overall risk for $\mathrm{MI}$ and cardiac death in the patients with previous DES-PCl, owing to higher MI rates but similar mortality risk. ${ }^{226}$ However, this difference was highly timedependent and limited to the first month after DES-PCI. ${ }^{226}$ These data suggest that surgery, if possible, should be delayed for at least 1 month after DES-PCl. Data for patients with coronary stents implanted in a Veterans' Administration (VA) hospital from 2000 to 2010 were also recently matched with VA Surgical Quality Improvement Program data to identify non-cardiac surgery within 24 months of stent placement. ${ }^{227}$ Each patient with a stent(s) was matched with two surgical patients without stents on surgical characteristics and cardiac risk factors. The two groups had similar risk of adverse cardiac events during 2 years of follow-up. However, patients with stents had a higher risk of adverse cardiac events within the 30-day post-operative period. ${ }^{227}$ The incremental risk did not vary by stent type. ${ }^{227}$ In both studies, roughly $50 \%$ of patients underwent stenting because of ACS and no incremental risk was observed in this higher risk population as compared to stable CAD patients.

Therefore, a minimum of 1 month of DAPT should be considered, independently of the type of implanted stent (i.e. BMS or newer-generation DES), in cases when surgery cannot be delayed for a longer period; however, such surgical procedures should be performed in hospitals where catheterization laboratories are available $24 / 7$, so as to treat patients immediately in case of perioperative thrombotic events (Figure 8). In patients at high ischaemic risk due to ACS presentation or complex coronary revascularization procedure, delaying surgery up to 6 months after index ACS or $\mathrm{PCl}$ may be reasonable as an additional safeguard to minimize the risk of perisurgical $\mathrm{MI}$, and based on unmatched retrospective registry data if the risks of further delaying surgery are acceptable.

In patients needing surgery within a few days, it was previously recommended to withhold clopidogrel and ticagrelor for 5 days and prasugrel for 7 days prior to surgery unless there is a high risk of thrombosis. ${ }^{228}$ However, emerging evidence, which is extensively discussed in Chapter 5, challenges such a long discontinuation period for ticagrelor before a safe surgical procedure can be undertaken (Figure 9). ${ }^{152,153}$

Although these data refer to patients undergoing cardiac surgery, it is rational to extend these findings to the non-cardiac surgery population, given the same offset kinetics and principally lower risk of bleeding in non-cardiac surgeries relative to cardiac surgery procedures (Figure 9). In cases where the consequences of even minor bleeding would be unacceptable (e.g. spinal surgery or other neurosurgical procedures) or the bleeding risk largely outweighs the ischaemic risk (e.g. a medium- to high-risk surgical bleeding procedure is undertaken 6 months or more after single stent implantation for stable CAD indication), P2Y 12 inhibitors may be discontinued for a 


\section{$\mathbf{P 2 Y}_{12}$ inhibitor interruption after $P C l$ for elective non-cardiac surgery'}

\section{$\downarrow$}

ACS at index $\mathrm{PCl}$ or other high ischaemic risk features? $?^{2}$

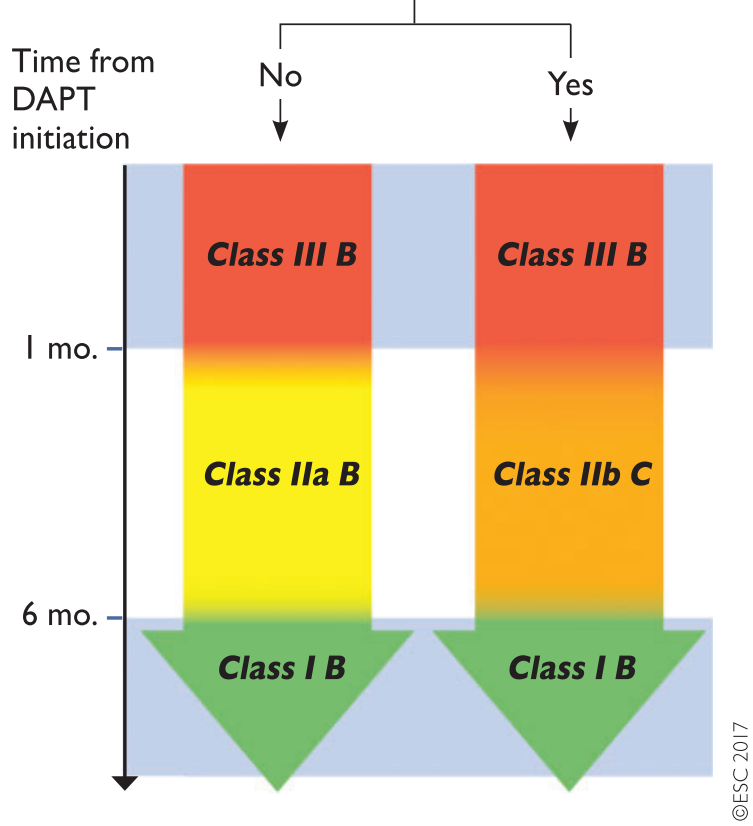

Figure 8 Timing for elective non-cardiac surgery in patients treated with dual antiplatelet therapy (DAPT) after percutaneous coronary intervention $(\mathrm{PCl})$. Colour-coding refers to the ESC Classes of Recommendations (green = Class I; yellow = lla; orange = Class Ilb).

ACS = acute coronary syndromes.

${ }^{1}$ Availability of $\mathrm{H} 24$ cath-lab service in place is suggested in case of major surgery within 6 months after $\mathrm{PCl}$.

${ }^{2} \mathrm{High}$ ischaemic risk features are presented in Table 5.

longer duration of time to ensure no residual platelet inhibition at the time of planned surgery. For patients with a very high risk of stent thrombosis, bridging therapy with intravenous, reversible glycoprotein inhibitors, such as eptifibatide or tirofiban, ${ }^{229}$ may be considered. Cangrelor, a reversible intravenous $\mathrm{P}_{2} \mathrm{Y}_{12}$ inhibitor, has been shown to provide effective platelet inhibition ${ }^{230}$ and is an appealing alternative to glycoprotein Ilb/Illa inhibitors, ${ }^{231}$ given the well-known role of $\mathrm{P}_{2} \mathrm{Y}_{12}$ inhibition in preventing stent thrombosis and the quicker offset of action as compared to tirofiban or eptifibatide. Concomitant parenteral anticoagulation therapy in conjunction with cangrelor or reversible glycoprotein inhibitors is not recommended to minimize bleeding risk while awaiting surgical procedures.

Restart after surgery: If $\mathrm{P}_{2} \mathrm{Y}_{12}$ inhibitor therapy has been stopped before a surgical procedure, therapy should be restarted as soon as possible (within $48 \mathrm{~h}$ ), given the substantial thrombotic hazard associated with lack of platelet inhibition early after surgery in patients with recent stent implantation and/or an ACS episode (Figure 9). ${ }^{232,233}$

The time for restarting $\mathrm{P}_{2} \mathrm{Y}_{12}$ inhibitors after surgery should ultimately be determined via a multidisciplinary discussion before surgery and traced in the patient file.

\section{Dual antiplatelet therapy in patients undergoing elec-} tive non-cardiac surgery

\begin{tabular}{|c|c|c|}
\hline Recommendations & Class $^{a}$ & Level $^{b}$ \\
\hline $\begin{array}{l}\text { It is recommended to continue aspirin peri- } \\
\text { operatively if the bleeding risk allows, and to } \\
\text { resume the recommended } \\
\text { antiplatelet therapy as soon as possible } \\
\text { post-operatively. }{ }^{232-236}\end{array}$ & I & B \\
\hline $\begin{array}{l}\text { After coronary stent implantation, elective } \\
\text { surgery requiring discontinuation of the } \\
\mathrm{P}_{2 Y_{12}} \text { inhibitor should be considered after } \\
1 \text { month, irrespective of the stent type, if } \\
\text { aspirin can be maintained throughout the } \\
\text { perioperative period. }{ }^{227}\end{array}$ & Ila & B \\
\hline $\begin{array}{l}\text { Discontinuation of } \mathrm{P}_{2} \mathrm{Y}_{12} \text { inhibitors should } \\
\text { be considered at least } 3 \text { days before surgery } \\
\text { for ticagrelor, at least } 5 \text { days for } \\
\text { clopidogrel, and at least } 7 \text { days for } \\
\text { prasugrel. }^{152,153,160}\end{array}$ & Ila & $\mathbf{B}$ \\
\hline $\begin{array}{l}\text { A multidisciplinary expert team should be } \\
\text { considered for pre-operative evaluation of } \\
\text { patients with an indication for DAPT before } \\
\text { elective surgery. }\end{array}$ & Ila & C \\
\hline $\begin{array}{l}\text { In patients with recent } \mathrm{Ml} \text { or other high } \\
\text { ischaemic risk features }{ }^{c} \text { requiring DAPT, } \\
\text { elective surgery may be postponed for up } \\
\text { to } 6 \text { months. } 17,214,215,234\end{array}$ & Ilb & C \\
\hline $\begin{array}{l}\text { If both oral antiplatelet agents have to be } \\
\text { discontinued perioperatively, a bridging } \\
\text { strategy with intravenous antiplatelet agents } \\
\text { may be considered, especially if surgery has } \\
\text { to be performed within } 1 \text { month after stent } \\
\text { implantation. }\end{array}$ & IIb & C \\
\hline $\begin{array}{l}\text { It is not recommended to discontinue } \\
\text { DAPT within the first month of treatment } \\
\text { in patients undergoing elective non-cardiac } \\
\text { surgery. }\end{array}$ & III & B \\
\hline
\end{tabular}

DAPT = dual antiplatelet therapy; $\mathrm{MI}=$ myocardial infarction

${ }^{\text {a }}$ Class of recommendation.

'Level of evidence.

'High ischaemic risk features are provided in Table 5. 


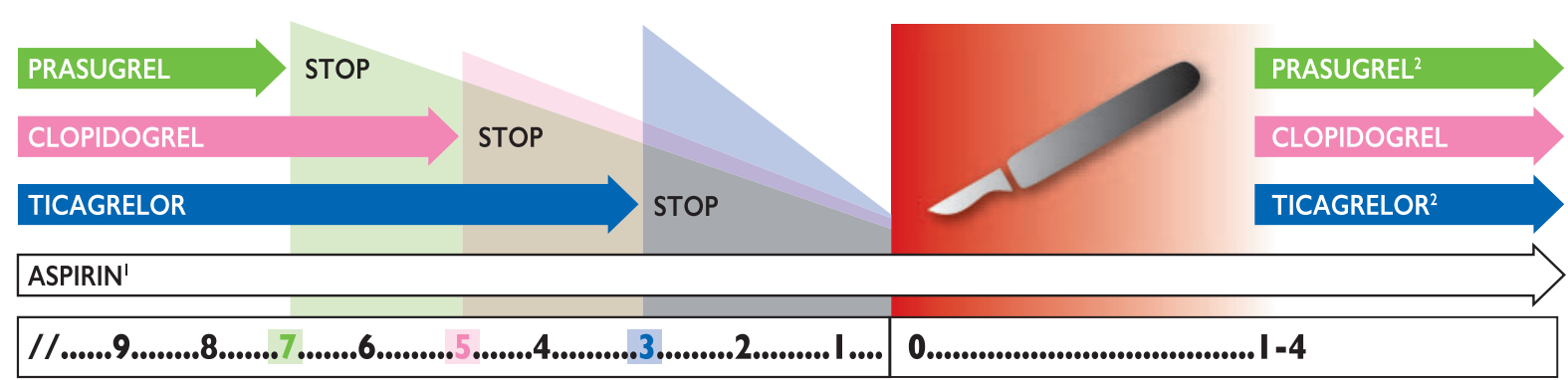

Minimal delay for $\mathrm{P}_{2} \mathrm{Y}_{12}$ interruption

Days after surgery

\footnotetext{
$=$ Expected average platelet function recovery

I Decision to stop aspirin throughout surgery should be made on a single case basis taking into account the surgical bleeding risk. 2 In patients not requiring $O A C$
}

Figure 9 Minimal discontinuation and re-implementation time frames of dual antiplatelet therapy (DAPT) for patients undergoing elective surgery $\mathrm{OAC}=$ oral anticoagulant.

\section{Gender consideration and special populations}

\subsection{Gender specificities}

There is no convincing evidence for a gender-related difference in the efficacy and safety of currently available DAPT type or duration across studies. No single trial or pooled analysis of investigations assessing a shorter than 1 year vs. at least 1 year DAPT duration has shown heterogeneous findings across genders. ${ }^{26,112,240,241}$ In the DAPT trial, there was a borderline quantitative interaction suggesting a lower relative treatment benefit for stent thrombosis reduction with prolonged DAPT in female as compared to male patients $\left(P_{\text {int }}=0.04\right){ }^{26}$ However, no such signal was apparent for MACCE $\left(P_{\text {int }}=0.46\right)$ or bleeding $\left(P_{\text {int }}=0.40\right)$ endpoints. Within the PEGASUS trial, there was no signal suggesting heterogeneity across the primary study endpoint with respect to gender $\left(P_{\text {int }}=0.84\right) .{ }^{29}$ On the other hand, there was a positive quantitative interaction $\left(P_{\text {int }}=0.03\right)$ suggesting that female patients may derive a relatively greater treatment benefit with respect to stroke prevention from prolonged treatment with aspirin and ticagrelor as compared to aspirin alone. However, no such signal was evident for cardiovascular death, Ml, or safety endpoints.

\subsection{Diabetes mellitus}

Patients with diabetes mellitus presenting with both stable and unstable CAD carry a worse prognosis in terms of short- and long-term risks of fatal and non-fatal ischaemic events, with enhanced platelet hyperactivity playing a putative causal role. In the CURE trial, patients with diabetes derived a similar treatment benefit from the addition of clopidogrel on top of aspirin as compared to patients without. ${ }^{40}$ No signal for greater treatment benefit was apparent in TRITON-TIMI 38 in patients with diabetes as compared to those without with respect to the study primary endpoint, and a consistent lack of heterogeneity signal with respect to diabetes mellitus was observed in the PLATO trial. $^{20,23}$ Hence, there is no convincing evidence that the presence of diabetes should affect decision making with respect to the choice of P2Y 12 inhibitors.

As it related to DAPT duration, the DAPT study found a slightly lower relative risk reduction for $\mathrm{Ml}$ endpoint in patients with diabetes as compared to those without diabetes $\left(P_{\text {int }}=0.02\right) .{ }^{242}$ However, there was no signal for heterogeneity with respect to the concomitant presence of diabetes mellitus across all other ischaemic or safety endpoints. Finally, no difference with respect to the presence or absence of diabetes was observed for the primary efficacy endpoint in the PEGASUS study $\left(P_{\text {int }}=0.99\right) .{ }^{145}$ Altogether, current evidence suggests that diabetes mellitus should not be the only appraised patient-specific feature when deciding upon the type or duration of DAPT.

\subsection{Lower-extremities artery disease}

Patients with LEAD are at heightened risk of ischaemic complications and mortality. The combination of symptomatic LEAD and CAD is associated with further heightened ischaemic risk beyond that associated with symptomatic disease in either vascular bed alone. ${ }^{243}$ In 3096 patients with LEAD included in the CHARISMA trial, DAPT was associated with a lower rate of $\mathrm{Ml}$ and hospitalization for ischaemic events but not the overall composite primary endpoint. There was no difference between the groups in moderate, severe, or fatal bleeding, but there was an increase in minor bleeding in the DAPT group. ${ }^{244}$ The PEGASUS investigators recently examined a subgroup of 1143 patients with LEAD and found that patients with prior MI with LEAD had a $60 \%$ increased risk of MACE relative to patients without LEAD, even after adjusting for differences in baseline characteristics. ${ }^{140}$ This increased ischaemic risk translated into a robust absolute risk reduction of $5.2 \%$ at 3 years with ticagrelor $60 \mathrm{mg}$ b.i.d. compared with placebo. In the setting of this robust ischaemic risk reduction, there were significant reductions in cardiovascular and all-cause mortality. Treatment with ticagrelor vs. placebo reduced the risk of adverse limb events in addition to the benefits observed for MACE and mortality. Reductions in acute limb ischaemia have also been shown with other antiplatelet 
Bleeding during treatment with dual antiplatelet therapy \pm OAC

\begin{tabular}{|c|c|c|}
\hline \\
\hline$\downarrow$ & $\downarrow$ & $\downarrow$ \\
\hline $\begin{array}{l}\text { TRIVIAL BLEEDING } \\
\text { Any bleeding not requiring } \\
\text { medical intervention or further } \\
\text { evaluation }\end{array}$ & $\begin{array}{l}\text { MILD BLEEDING } \\
\text { Any bleeding that requires } \\
\text { medical attention without } \\
\text { requiring hospitalization }\end{array}$ & $\begin{array}{l}\text { MODERATE BLEEDING } \\
\text { Any bleeding associated with a significant } \\
\text { blood loss }(>3 \mathrm{~g} / \mathrm{dL} \mathrm{HB}) \text { and/or requiring } \\
\text { hospitalization, which is haemodynamically }\end{array}$ \\
\hline $\begin{array}{l}\text { e.g. skin bruising or ecchimosis, } \\
\text { self-resolving epistaxis, minimal } \\
\text { conjunctival bleeding }\end{array}$ & $\begin{array}{l}\text { e.g. not self resolving epistaxis, } \\
\text { moderate conjunctival bleeding, } \\
\text { genitourinary or upper/lower } \\
\text { gastrointestinal bleeding without } \\
\text { significant blood loss, mild } \\
\text { haemoptysis }\end{array}$ & $\begin{array}{l}\text { e.g. genitourinary, respiratory or upper/lower } \\
\text { gastrointestinal bleeding with significant blood } \\
\text { loss or requiring transfusion }\end{array}$ \\
\hline - Continue DAPT & \multirow{3}{*}{$\begin{array}{l}\text { - Continue DAPT } \\
\text { - Consider shortening DAPT } \\
\text { duration or switching to less } \\
\text { potent } \mathrm{P}_{12} \mathrm{Y}_{12} \text { inhibitor } \\
\text { (i.e. from ticagrelor/prasugrel } \\
\text { to clopidogrel), especially } \\
\text { if recurrent bleeding occurs }\end{array}$} & \multirow{3}{*}{$\begin{array}{l}\text { - Consider stopping DAPT and continue with } \\
\text { SAPT, preferably with the } P 2 Y_{12} \text { inhibitor } \\
\text { especially in case of upper GI bleeding } \\
\text { - Reinitiate DAPT as soon as deemed safe } \\
\text { - Consider shortening DAPT duration or } \\
\text { switching to less potent } \mathrm{P} 2 \mathrm{Y}_{12} \text { inhibitor } \\
\text { (i.e. from ticagrelor/prasugrel to clopidogrel), } \\
\text { especially if recurrent bleeding occurs }\end{array}$} \\
\hline $\begin{array}{l}\text { - Consider OAC continuation } \\
\text { or skip one single next pill }\end{array}$ & & \\
\hline \multirow{5}{*}{$\begin{array}{l}\text { - Reassure the patient } \\
\text { - Identify and discuss with the } \\
\text { patient possible preventive } \\
\text { strategies } \\
\text { - Counsel patient on the } \\
\text { importance of drug-adherence }\end{array}$} & & \\
\hline & $\begin{array}{l}\text { - In case of triple therapy } \\
\text { consider downgrading to } \\
\text { dual therapy, preferably with } \\
\text { clopidogrel and OAC }\end{array}$ & \multirow{3}{*}{$\begin{array}{l}\text { - Consider OAC discontinuation or even } \\
\text { reversal until bleeding is controlled, unless } \\
\text { very high thrombotic risk (i.e. mechanical } \\
\text { heart valves, cardiac assist device, } \\
\mathrm{CHA}_{2} \mathrm{DS}_{2} \text {-VASc } \geq 4 \text { ) } \\
\text { - Reinitiate treatment within one week if } \\
\text { clinically indicated. For Vitamin-K antagonist } \\
\text { consider a target INR of } 2.0-2.5 \text { unless } \\
\text { overriding indication (i.e. mechanical heart } \\
\text { valves or cardiac assist device) for NOAC } \\
\text { consider the lowest effective dose } \\
\text { - In case of triple therapy consider downgrading } \\
\text { to dual therapy, preferably with clopidogrel } \\
\text { and OAC } \\
\text { - If patients on dual therapy, consider stopping } \\
\text { antiplatelet therapy if deemed safe }\end{array}$} \\
\hline & $\begin{array}{l}\text { - Identify and possibly treat } \\
\text { concomitant conditions } \\
\text { associated with bleeding } \\
\text { (e.g. peptic ulcer, haemorrhoidal } \\
\text { plexus, neoplasm) } \\
\text { - Add PPI if not previously } \\
\text { implemented } \\
\text { - Counsel patient on the } \\
\text { importance of drug-adherence }\end{array}$ & \\
\hline & & \\
\hline & & \multirow{5}{*}{$\begin{array}{l}\text { - Consider i.v. PPI if GI bleeding occurred } \\
\text { - Identify and possibly treat concomitant } \\
\text { conditions associated with bleeding } \\
\text { (e.g. peptic ulcer, haemorrhoidal plexus, } \\
\text { neoplasm) } \\
\text { - Counsel patient on the importance of } \\
\text { drug-adherence }\end{array}$} \\
\hline Legend & & \\
\hline DAPT management & & \\
\hline $\mathrm{OAC}$ management & & \\
\hline General recommendations & & \\
\hline
\end{tabular}

Figure I 0 Practical recommendations for the management of bleeding in patients treated with dual antiplatelet therapy with or without concomitant oral anticoagulation. Practical recommendations for the management of bleeding in patients treated with dual antiplatelet therapy with or without concomitant oral anticoagulation. Blue boxes refer to management of antiplatelet therapy. Dark-red boxes refer to the management of oral anticoagulation. Light-green boxes refer to general recommendation for patients' safety.

$\mathrm{ACS}=$ acute coronary syndrome; $\mathrm{CHA}_{2} \mathrm{DS}_{2}-\mathrm{VASc}=$ cardiac failure, hypertension, age $\geq 75$ (2 points), diabetes, stroke (2 points)-vascular disease, age 65-74, sex category; DAPT = dual antiplatelet therapy; GI = gastrointestinal; HB = haemoglobin; INR = international normalized ratio; i.v. = intravenous; $\mathrm{OAC}=$ oral anticoagulant; $\mathrm{NOAC}=$ non-vitamin-K antagonist; $\mathrm{PPI}=$ proton pump inhibitor; $\mathrm{RBC}$ = red blood cell; SAPT $=$ single antiplatelet therapy. 


\section{Bleeding during treatment with dual antiplatelet therapy \pm OAC}

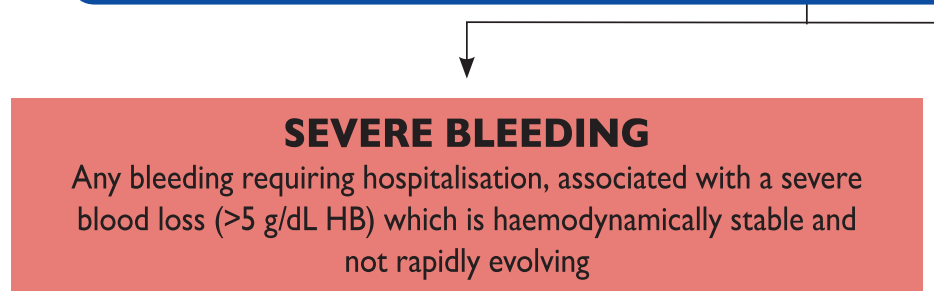

e.g. severe genitourinary, respiratory or upper/lower gastrointestinal bleeding

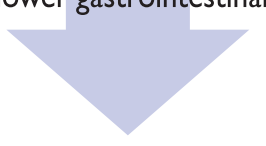

- Consider stopping DAPT and continue with SAPT, preferably with the $\mathrm{P}_{2} \mathrm{Y}_{12}$ inhibitor especially in case of upper GI bleeding

- If bleeding persists despite treatment or treatment is not possible, consider stopping all antithrombotic medications

- Once bleeding has ceased, re-evaluate the need for DAPT or SAPT, preferably with the $\mathrm{P}_{2} \mathrm{Y}_{12}$ inhibitor especially in case of upper $\mathrm{GI}$ bleeding

- If DAPT is re-started, consider shortening DAPT duration or switching to less potent $\mathrm{P}_{2} \mathrm{Y}_{12}$ inhibitor (i.e. from ticagrelor/ prasugrel to clopidogrel), especially if recurrent bleeding occurs

- Consider stopping and reversing OAC until bleeding is controlled unless prohibitive thrombotic risk (i.e. mechanical heart valve in mitral position, cardiac assist device)

- Reinitiate treatment within one week if clinically indicated. For vitamin-K antagonists consider a target INR of 2.0-2.5 unless overriding indication (i.e. mechanical heart valves or cardiac assist device) for NOAC consider the lowest effective dose

- If patient on triple therapy consider downgrading to dual therapy with clopidogrel and OAC. If patients on dual therapy, consider stopping antiplatelet therapy if deemed safe

- Consider i.v. PPI if GI bleeding occurred

- RBC transfusion if HB $<7-8 \mathrm{~g} / \mathrm{dL}$

- Consider platelet transfusion

- Urgent surgical or endoscopic treatment of bleeding source if deemed possible

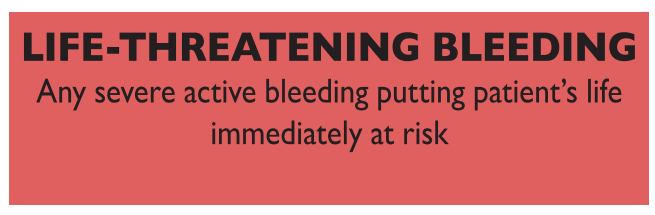

e.g. massive overt genitourinary, respiratory or upper/lower gastrointestinal bleeding, active intracranial, spinal or intraocular haemorrhage, or any bleeding causing haemodynamic instability

- Immediately discontinue all antithrombotic medications

- Once bleeding has ceased, re-evaluate the need for DAPT or SAPT, preferably with the $\mathrm{P}_{2} \mathrm{Y}_{12}$ inhibitor especially in case of upper $\mathrm{GI}$ bleeding

\section{- Stop and reverse OAC}

- Fluid replacement if hypotension

- Consider RBC transfusion irrespective of $\mathrm{HB}$ values

- Platelet transfusion

- Consider i.v. PPI if GI bleeding occurred

- Urgent surgical or endoscopic treatment of bleeding source if deemed possible

Figure 10 Continued

agents, such as vorapaxar, demonstrating that this morbidity is modifiable with potent and prolonged antithrombotic strategies. ${ }^{245}$ In the allcomer PRODIGY trial, 246 (12.5\%) patients were included with symptomatic LEAD. LEAD status was associated with a higher risk of death and ischaemic events (HR 2.80, 95\% Cl 2.05-3.83; $P<0.001){ }^{246}$
Prolonged vs. short DAPT conveyed a lower risk of the primary efficacy endpoint in LEAD patients (16.1\% vs. $27.3 \%$; $\mathrm{HR} 0.54,95 \% \mathrm{Cl}$ $0.31-0.95 ; P=0.03)$ but not in patients without LEAD (9.3\% vs. 7.4\%; HR 1.28, 95\% Cl 0.92-1.77; $P=0.14)$, with positive interaction ( $P=$ 0.01). The risk of definite or probable stent thrombosis as well as 
overall mortality was significantly lower in LEAD patients treated with prolonged DAPT as compared with those receiving short DAPT.

\subsection{Complex percutaneous coronary intervention}

While high $\mathrm{PCl}$ complexity intuitively represents a driver for favouring a prolonged over a shortened DAPT duration, the evidence regarding optimal DAPT duration based on complexity of intervention is limited. In a patient-level meta-analysis from six RCTs investigating DAPT durations after coronary stenting, including 9577 patients, complex $\mathrm{PCl}$ was defined as the composite of at least three stents implanted, at least three lesions treated, bifurcation with two stents implanted, total stent length $>60 \mathrm{~mm}$, and chronic total occlusion as target lesion. ${ }^{247}$ Patients who underwent complex $\mathrm{PCl}$ had a two-fold increase of MACE (5.0\% vs. $2.5 \% ; P=0.001)$. Long- and short-DAPT were defined as a DAPT duration $\geq 12$ months and $\leq 6$ months, respectively. Compared with short-DAPT, long-DAPT was associated with a significant reduction in MACE in the complex $\mathrm{PCl}$ group (4.0\% vs. $6.0 \%$; adjusted $\mathrm{HR} 0.56,95 \% \mathrm{Cl} 0.35-0.89)$ vs. the non-complex $\mathrm{PCl}$ group (2.5\% vs. $2.6 \%$; adjusted $\left.\mathrm{HR} 1.01,95 \% \mathrm{Cl} 0.75-1.35 ; P_{\text {int }}=0.01\right)$. The magnitude of the reduction in MACE with long-DAPT increased progressively as the degree of procedural complexity was greater. LongDAPT was overall associated with increased risk of major bleeding, which was uniform in magnitude between groups $\left(P_{\text {int }}=0.15\right)$.

\subsection{Dual antiplatelet therapy decision making in patients with stent thrombosis}

Patients presenting with stent thrombosis represent a challenging patient population in whom no randomized clinical evidence is available to guide decision making. Observational studies have shown that the risk of stent thrombosis recurrence after the first episode of stent thrombosis is worrisome. Armstrong et al reported on a combined retrospective and prospective observational California registry of angiographic definite stent thrombosis at five academic hospitals from 2005 to $2013 .^{248}$ The entry criterion was the occurrence of a definite stent thrombosis, which was observed in 221 patients overall out of an unknown number of patients at risk. With the important caveat of not knowing for each stent type the exact timing of the first stent thrombosis event after the index procedure, 104 (47\%) patients had received a first generation DES, 51 (23\%) a BMS, and 19 (9\%) a second generation DES. After a median follow-up of 3.3 years, 29 patients developed definite or probable recurrent stent thrombosis, while 19 presented angiographic definite recurrent stent thrombosis. The cumulative hazard of definite or probable recurrent stent thrombosis was $16 \%$ at 1 year and $24 \%$ at 5 years. The cumulative hazard of angiographic definite recurrent stent thrombosis was $11 \%$ at 1 year and $20 \%$ at 5 years. Taken together, these findings confirm the high risk of stent thrombosis recurrence after the first stent thrombosis. An additional piece of information, which is conveyed by this important analysis, is that the risk of recurrence is highest in the first few months after the first event and that it does not abate entirely over time. Both prasugrel and ticagrelor have been shown to be associated with a significant reduction of definite and definite or probable stent thrombosis as compared to clopidogrel. ${ }^{20,23}$ Moreover, both studies indicated that the number of recurrent events is also significantly decreased by treatment with ticagrelor or prasugrel as compared to clopidogrel. Hence, the use of clopidogrel after stent thrombosis cannot be regarded as an effective treatment option. Considering the long-term risk of recurrence after first stent thrombosis, it may be reasonable to make every effort to maintain DAPT for a very long-term period in this highly selected high-risk patient population, if tolerated.

\subsection{Patients who develop bleeding while on treatment}

Patients who develop bleeding complications while on DAPT represent a challenging patient population for whom no guidance from RCTs is available.

The decision to withhold or continue DAPT in this setting largely depends on ischaemic (e.g. indication for DAPT and time from last stent insertion, if any, to bleeding) vs. recurrent/prolonged bleeding risks. A practical flow chart in order to manage this challenging population is provided in Figure 10 and additional information on practical management can be found elsewhere. ${ }^{249}$ As bleeding is an independent predictor of recurrent bleeding, ${ }^{250}$ type, dose, and duration of DAPT should be reassessed in this setting.

\section{Gender considerations and special populations}

\begin{tabular}{|l|c|c|}
\hline Recommendations & Class $^{\mathrm{a}}$ & Level $^{\mathrm{b}}$ \\
\hline $\begin{array}{l}\text { Similar type and duration of DAPT are rec- } \\
\text { ommended in male and female } \\
\text { patients. }\end{array}$.6,240 & I & A \\
\hline $\begin{array}{l}\text { It is recommended to reassess the type, } \\
\text { dose, and duration of DAPT in patients with } \\
\text { actionable bleeding complications while on } \\
\text { treatment. }\end{array}$ & I & C \\
\hline $\begin{array}{l}\text { Similar type and duration of DAPT should } \\
\text { be considered in patients with and without } \\
\text { diabetes mellitus. }{ }^{14,242}\end{array}$ & Ila & B \\
\hline $\begin{array}{l}\text { Prolonged (i.e. }>12 \text { months } \\
\text { tion } \text { ) DAPT dura- } \\
\text { prior stent thrombosis, especially in the } \\
\text { absence of correctable causes (e.g. lack of } \\
\text { adherence or correctable mechanical stent- } \\
\text { related issues). }\end{array}$ & Ila & C \\
\hline $\begin{array}{l}\text { Prolonged (i.e. }>12 \text { months) DAPT duration } \\
\text { may be considered in CAD patients with } \\
\text { LEAD. }{ }^{140,246}\end{array}$ & Ilb & B \\
\hline $\begin{array}{l}\text { Prolonged (i.e. }>6 \text { months) DAPT duration } \\
\text { may be considered in patients who under- } \\
\text { went complex PCl. }\end{array}$ & Ilb & B \\
\hline
\end{tabular}

$\mathrm{CAD}=$ coronary artery disease; DAPT $=$ dual antiplatelet therapy; $\mathrm{LEAD}=$ lower-extremities artery disease; $\mathrm{PCl}=$ percutaneous coronary intervention. ${ }^{\mathrm{a} C}$ Class of recommendation.

bLevel of evidence.

'Possibly for as long as can be tolerated.

${ }^{d}$ Complex $\mathrm{PCl}$ defined as the composite of at least three stents implanted, at least three lesions treated, bifurcation with two stents implanted, total stent length $>60 \mathrm{~mm}$, and chronic total occlusion as target lesion. 


\section{Key messages}

(1) Benefits and risks of DAPT: DAPT reduces the risk of stent thrombosis across the entire spectrum of events, from acute to very late occurrences. However, treatment with DAPT beyond 1 year after $\mathrm{MI}$, or after $\mathrm{PCl}$, exerts the majority of its benefit by reducing the rate of spontaneous MI. The risk of bleeding in patients on DAPT is proportionally related to its duration both within and beyond 1 year of treatment duration. Since the benefits of prolonged DAPT, especially for mortality endpoints, appear highly dependent on prior cardiovascular history (such as prior ACS/MI vs. stable CAD), and prediction models to estimate on-DAPT bleeding risk have been developed, an individualized approach based on ischaemic vs. bleeding risk assessment is warranted.

(2) Bleeding mitigation strategy: Every effort should be pursued to mitigate the risk of bleeding complications while the patient is on DAPT, including access site selection, modulation of modifiable risk factors for bleeding, low dose aspirin, low dose of P2Y12 inhibitor as appropriate, and routine use of PPI.

(3) $\mathrm{P}_{2} Y_{12}$ inhibitor selection: Clopidogrel is considered the default $\mathrm{P} \mathrm{Y}_{12}$ inhibitor in patients with stable CAD treated with $\mathrm{PCl}$, those with indication to concomitant oral anticoagulation, as well as in ACS patients in whom ticagrelor or prasugrel are contraindicated. Ticagrelor or prasugrel is recommended in ACS patients unless drug-specific contraindications exist.

(4) Timing of $P_{2} Y_{12}$ inhibitor initiation: The timing of initiation of a $\mathrm{P}^{2} \mathrm{Y}_{12}$ inhibitor is both drug- (i.e. ticagrelor or clopidogrel vs. prasugrel) and disease-specific (i.e. SCAD vs. ACS and type thereof).

(5) Stable CAD patients treated with PCl: Irrespective of the type of metallic stent implanted, the duration of DAPT is $1-6$ month(s) depending on the bleeding risk. For patients in whom the ischaemic risk prevails over the risk of bleeding, a longer DAPT duration may be considered.

(6) Metallic stent type and DAPT duration: The need for a short DAPT regimen should no longer justify the use of BMS instead of newer-generation DES. DAPT duration in each individual patient should be guided by an individualized approach based on ischaemic vs. bleeding risk assessment and not by the stent type.

(7) Stable CAD patients treated with CABG: There is insufficient data to recommend DAPT in this patient population.

(8) ACS patients: Irrespective of the final revascularization strategy (e.g. medical therapy, $\mathrm{PCl}$, or $\mathrm{CABG}$ ), the default DAPT duration in these patients is 12 months. Six-month therapy duration should be considered in high bleeding risk patients, whereas $>12$-month therapy may be considered in ACS patients who have tolerated DAPT without a bleeding complication.

(9) Patients with indication for oral anticoagulation: Compared with OAC therapy alone, the addition of DAPT to OAC therapy results in at least a two- to three-fold increase in bleeding complications. Therefore, these patients should be considered at high risk of bleeding and the indication for OAC should be reassessed and treatment continued only if a compelling indication exists. The duration of triple therapy should be limited up to a maximum of 6 months or omitted after hospital discharge, taking into account the ischaemic (e.g. complexity of treated CAD, amount of disease left untreated, technical considerations regarding stent implantation techniques, and results) as well as the bleeding risk. The use of ticagrelor or prasugrel in this setting is not recommended.

(10) Patients undergoing elective non-cardiac surgery after coronary stent implantation: A multidisciplinary expert team should be considered for pre-operative evaluation of patients with an indication for DAPT before elective surgery. Scheduled surgery requiring discontinuation of the $\mathrm{P} 2 \mathrm{Y}_{12}$ inhibitor should be considered after at least 1 month, irrespective of the stent type, if aspirin can be maintained throughout the perioperative period. If both oral antiplatelet agents have to be discontinued perioperatively, a bridging strategy with cangrelor, tirofiban, or eptifibatide may be considered, especially if surgery has to be performed within 1 month after stent implantation.

(11) Gender consideration and special populations: Similar type and duration of DAPT are recommended in male and female patients, as well as in patients with and without diabetes mellitus. Patients with prior stent thrombosis, especially in the absence of correctable causes, should receive prolonged DAPT. A prolonged DAPT regimen may also be considered in patients with LEAD or who have undergone complex $\mathrm{PCl}$. It is recommended to reassess the type, dose, and duration of DAPT in patients with actionable bleeding complications while on treatment. In patients with active bleeding while on DAPT, the decision to stop both antiplatelet agents, especially if shortly after PCl, should be taken only if the bleeding is life-threatening and the source has not been or cannot be treated. In such a rare case scenario, the patient should be transferred to a primary PCI facility centre.

\section{Evidenced-based 'to do and not to do' messages}

\section{Recommendations that are class I or III with a level of evidence A or B}

\begin{tabular}{|c|c|c|}
\hline Recommendations on $\mathrm{P}_{2} \mathrm{Y}_{12}$ inhibitor selection and timing & Class $^{\mathrm{a}}$ & Level $^{\mathrm{b}}$ \\
\hline $\begin{array}{l}\text { In patients with ACS, ticagrelor ( } 180 \mathrm{mg} \text { loading dose, } 90 \mathrm{mg} \text { b.i.d.) on top of aspirin is recommended, regardless of initial } \\
\text { treatment strategy, including patients pre-treated with clopidogrel (which should be discontinued when ticagrelor is com- } \\
\text { menced) unless there are contraindications. }{ }^{c}\end{array}$ & $\mathbf{I}$ & B \\
\hline $\begin{array}{l}\text { In patients with ACS undergoing PCl, prasugrel }\left(60 \mathrm{mg} \text { loading dose, } 10 \mathrm{mg} \text { o.d.) on top of aspirin is recommended for } \mathrm{P} 2 \mathrm{Y}_{12} \text { inhibi- }\right. \\
\text { tor-naive patients with NSTE-ACS or initially conservatively managed STEMI if indication for PCl is established, or in STEMl patients } \\
\text { undergoing immediate coronary catheterization unless there is a high risk of life-threatening bleeding or other contraindications. }{ }^{c}\end{array}$ & $\mathbf{I}$ & B \\
\hline
\end{tabular}




\begin{tabular}{|c|c|c|}
\hline $\begin{array}{l}\text { Pre-treatment with a } \mathrm{P}_{2} \mathrm{Y}_{12} \text { inhibitor is generally recommended in patients in whom coronary anatomy is known and the } \\
\text { decision to proceed to } \mathrm{PCl} \text { is made, as well as in patients with STEMI. }\end{array}$ & $\mathbf{I}$ & A \\
\hline $\begin{array}{l}\text { Clopidogrel ( } 600 \mathrm{mg} \text { loading dose, } 75 \mathrm{mg} \text { o.d.) on top of aspirin is recommended in stable CAD patients undergoing } \\
\text { coronary stent implantation and in ACS patients who cannot receive ticagrelor or prasugrel, including those with prior } \\
\text { intracranial bleeding or indication for OAC. }\end{array}$ & I & $\mathbf{A}$ \\
\hline $\begin{array}{l}\text { Clopidogrel ( } 300 \mathrm{mg} \text { loading dose in patients aged } \leq 75,75 \mathrm{mg} \text { o.d.) is recommended on top of aspirin in STEMI patients } \\
\text { receiving thrombolysis. }\end{array}$ & $\mathbf{I}$ & A \\
\hline In NSTE-ACS patients in whom coronary anatomy is not known, it is not recommended to administer prasugrel. & III & B \\
\hline \multicolumn{3}{|l|}{ Measures to minimize bleeding while on dual antiplatelet therapy } \\
\hline Radial over femoral access is recommended for coronary angiography and $\mathrm{PCl}$ if performed by an expert radial operator. & $\mathbf{I}$ & A \\
\hline In patients treated with DAPT, a daily aspirin dose of $75-100 \mathrm{mg}$ is recommended. & $\mathbf{I}$ & A \\
\hline A PPI in combination with DAPT is recommended. ${ }^{d}$ & $\mathbf{I}$ & B \\
\hline Routine platelet function testing to adjust antiplatelet therapy before or after elective stenting is not recommended. & III & A \\
\hline \multicolumn{3}{|l|}{ Switching between oral $\mathbf{P} 2 Y_{12}$ inhibitors } \\
\hline $\begin{array}{l}\text { In patients with ACS who were previously exposed to clopidogrel, switching from clopidogrel to ticagrelor is } \\
\text { recommended early after hospital admission at a loading dose of } 180 \mathrm{mg} \text { irrespective of timing and loading dose of } \\
\text { clopidogrel, unless contraindications to ticagrelor exist. }{ }^{\text {c }}\end{array}$ & I & B \\
\hline
\end{tabular}

\title{
Dual antiplatelet therapy duration in patients with acute coronary syndrome treated with percutaneous coronary intervention
}

In patients with ACS treated with coronary stent implantation, DAPT with a P2Y 12 inhibitor on top of aspirin is recommended for 12 months unless there are contraindications such as excessive risk of bleeding (e.g. PRECISE-DAPT $\geq 25$ ).

\section{Dual antiplatelet therapy duration in patients with acute coronary syndrome undergoing medical therapy management}

\begin{abstract}
In patients with ACS who are managed with medical therapy alone and treated with DAPT, it is recommended to continue $\mathrm{P}_{2} \mathrm{Y}_{12}$ inhibitor therapy (either ticagrelor or clopidogrel) for 12 months.
\end{abstract}

Ticagrelor is recommended over clopidogrel, unless the bleeding risk outweighs the potential ischaemic benefit.

Prasugrel is not recommended in medically managed ACS patients.

\section{Dual antiplatelet therapy in patients undergoing elective cardiac and non-cardiac surgery}

It is recommended to continue aspirin perioperatively if the bleeding risk allows, and to resume the recommended antiplatelet therapy as soon as possible post-operatively.

It is not recommended to discontinue DAPT within the first month of treatment in patients undergoing elective non-cardiac surgery.

\begin{tabular}{|c|c|c|}
\hline I & A \\
\hline I & B \\
\hline III & B \\
\hline
\end{tabular}

\section{Gender considerations}

Similar type and duration of DAPT are recommended in male and female patients.

ACS = acute coronary syndrome; CAD = coronary artery disease; DAPT = dual antiplatelet therapy; NSTE-ACS = non-ST elevation acute coronary syndrome; OAC = oral anticoagulant; $\mathrm{PCl}=$ percutaneous coronary intervention; PPI = proton pump inhibitor; PRECISE-DAPT = PREdicting bleeding Complications In patients undergoing Stent implantation and subsEquent Dual Anti Platelet Therapy; STEMI = ST-elevation myocardial infarction; TIA = transient ischaemic attack.

${ }^{a}$ Class of recommendation.

'Level of evidence.

${ }^{c}$ Contraindications for ticagrelor: previous intracranial haemorrhage or ongoing bleeds. Contraindications for prasugrel: previous intracranial haemorrhage, previous ischaemic stroke or TIA, or ongoing bleeds; prasugrel is not recommended for patients $\geq 75$ years of age or with a body weight $<60 \mathrm{~kg}$.

dWhile the evidence that a PPI does not increase the risk of cardiovascular events was generated with omeprazole, based on drug-drug interaction studies, omeprazole and esomeprazole would appear to have the highest propensity for clinically relevant interactions, while pantoprazole and rabeprazole have the lowest. 


\section{What is new in the 2017 ESC focussed update on DAPT?}

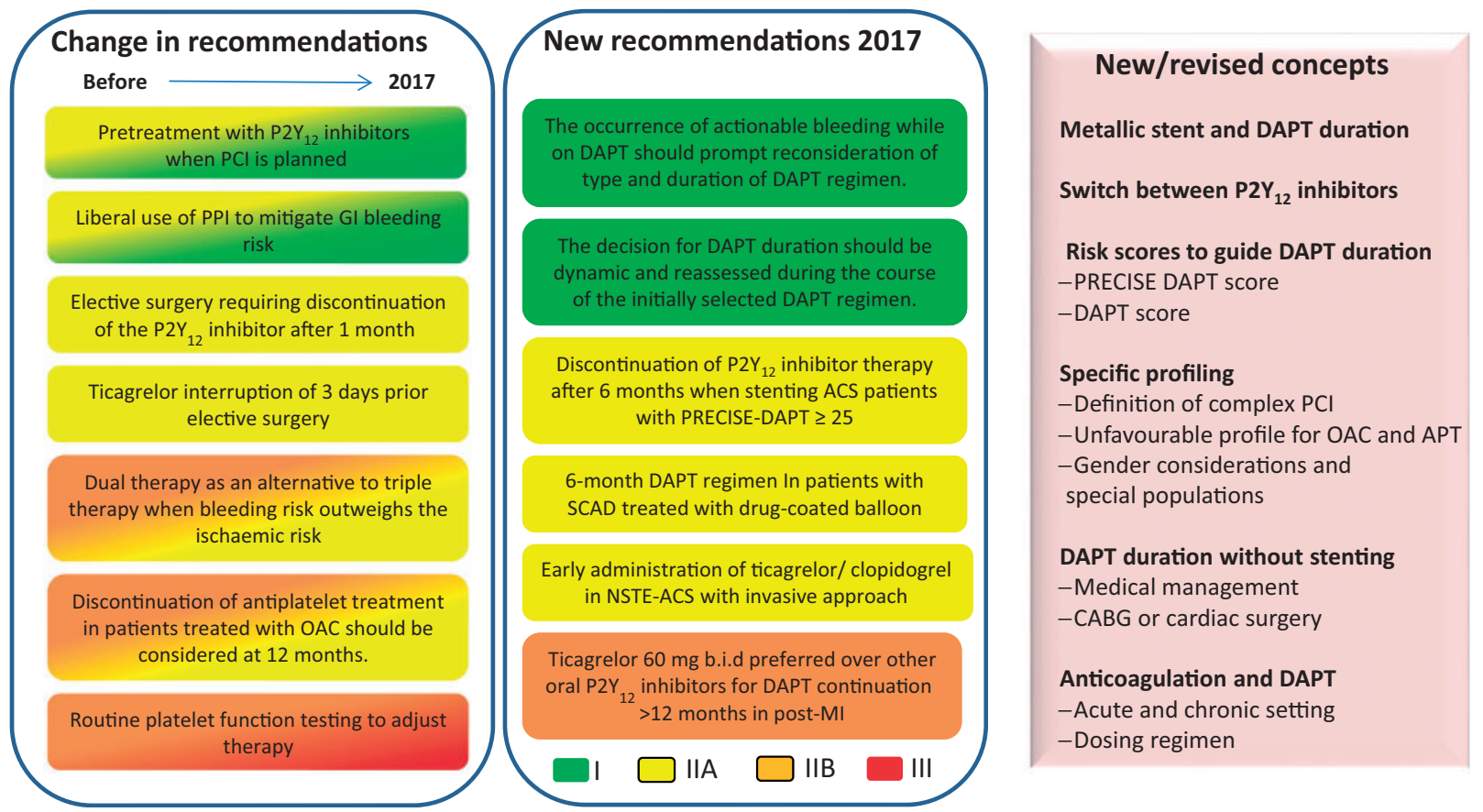

ACS $=$ acute coronary syndrome; APT $=$ anti-platelet therapy; $C A B G=$ coronary artery bypass graft; DAPT $=$ dual antiplatelet therapy; $\mathrm{MI}=$ myocardial infarction; NSTE $=$ Non-st-segment elevation; $\mathrm{OAC}=$ oral anti-coagulant; $\mathrm{PCl}=$ percuatenous coronary intervention; PRECISE-DAPT = PREdicting bleeding Complications In patients undergoing Stent implantation and subsEquent Dual Anti Platelet Therapy; Stable CAD = stable coronary artery disease.

\section{Web addenda and Clinical Cases companion document}

All Web figures, Web tables, and the Clinical Cases companion document are available at the European Heart Journal online and also via the ESC Web site at: www.escardio.org/guidelines

\section{Appendix}

ESC Committee for Practice Guidelines (CPG): Stephan Windecker (Chairperson) (Switzerland), Victor Aboyans (France), Stefan Agewall (Norway), Emanuele Barbato (Italy), Héctor Bueno (Spain), Antonio Coca (Spain), Jean-Philippe Collet (France), loan Mircea Coman (Romania), Veronica Dean (France), Victoria Delgado (The Netherlands), Donna Fitzsimons (UK), Oliver Gaemperli (Switzerland), Gerhard Hindricks (Germany), Bernard lung (France), Peter Jüni (Canada), Hugo A. Katus (Germany), Juhani Knuuti (Finland), Patrizio Lancellotti (Belgium), Christophe Leclercq (France), Theresa McDonagh (UK), Massimo Francesco Piepoli (Italy), Piotr Ponikowski (Poland), Dimitrios J. Richter (Greece), Marco Roffi (Switzerland), Evgeny Shlyakhto (Russia), lain A. Simpson (UK), and Jose Luis Zamorano (Spain)

ESC National Cardiac Societies actively involved in the review process of the 2017 ESC focused update on dual antiplatelet therapy in coronary artery disease in collaboration with EACTS:

Austria: Austrian Society of Cardiology, Franz Xaver Roithinger; Azerbaijan: Azerbaijan Society of Cardiology, Farid Aliyev; Belarus: Belorussian Scientific Society of Cardiologists, Valeriy Stelmashok; Belgium: Belgian Society of Cardiology, Walter Desmet; Bulgaria: Bulgarian Society of Cardiology, Arman Postadzhiyan; Cyprus: Cyprus Society of Cardiology, Georgios P. Georghiou; Czech Republic: Czech Society of Cardiology, Zuzana Motovska; Denmark: Danish Society of Cardiology, Erik Lerkevang Grove; Estonia: Estonian Society of Cardiology, Toomas Marandi; Finland: Finnish Cardiac Society, Tuomas Kiviniemi; The Former Yugoslar Republic of Macedonia: Macedonian Society of Cardiology, Sasko Kedev; France: French Society of Cardiology, Martine Gilard; Germany: German Cardiac Society, Steffen Massberg; Greece: Hellenic Society of Cardiology, Dimitrios Alexopoulos; Hungary: Hungarian Society of Cardiology, Robert Gabor Kiss; Iceland: Icelandic Society of Cardiology, Ingibjorg Jona Gudmundsdottir; Ireland: Irish Cardiac Society, Eugène P. McFadden; Israel: Israel Heart Society, Eli Lev; Italy: Italian Federation of Cardiology, Leonardo De Luca; Kazakhstan: Association of Cardiologists of Kazakhstan, Akhmetzhan Sugraliyev; Kosovo: Kosovo Society of Cardiology, Edmond Halit; Kyrgyzstan: Kyrgyz Society of Cardiology, Erkin Mirrakhimov, Latvia: Latvian Society of Cardiology, Gustavs Latkovskis; 
Lithuania: Lithuanian Society of Cardiology, Birute Petrauskiene; Luxembourg: Luxembourg Society of Cardiology, Steve Huijnen; Malta: Maltese Cardiac Society, Caroline Jane Magri; Morocco: Moroccan Society of Cardiology, Rhizlan Cherradi; The Netherlands: Netherlands Society of Cardiology, Jurrien M Ten Berg; Norway: Norwegian Society of Cardiology, Jan Eritsland; Poland: Polish Cardiac Society, Andrzej Budaj; Portugal: Portuguese Society of Cardiology, Carlos Tavares Aguiar; Russian Federation: Russian Society of Cardiology, Dmitry Duplyakov; San Marino: San Marino Society of Cardiology, Marco Zavatta; Serbia: Cardiology Society of Serbia, Nebojsa M. Antonijevic; Slovakia: Slovak Society of Cardiology, Zuzana Motovska; Slovenia: Slovenian Society of Cardiology, Zlatko Fras; Spain: Spanish Society of Cardiology, Antonio Tello Montoliu; Sweden: Swedish Society of Cardiology, Christoph Varenhorst; Switzerland: Swiss Society of Cardiology, Dimitri Tsakiris; Tunisia: Tunisian Society of Cardiology and Cardio-Vascular Surgery, Faouzi Addad; Turkey: Turkish Society of Cardiology, Sinan Aydogdu; Ukraine: Ukrainian Association of Cardiology, Alexander Parkhomenko; United Kingdom: British Cardiovascular Society, Tim Kinnaird.

\section{References}

1. Population Division. Department of Economic and Social Affairs. United Nations. Revision of World Population Prospects. https://esa.un.org/unpd/wpp/.

2. Schomig A, Neumann FJ, Kastrati A, Schuhlen H, Blasini R, Hadamitzky M, Walter H, Zitzmann-Roth EM, Richardt G, Alt E, Schmitt C, Ulm K. A randomized comparison of antiplatelet and anticoagulant therapy after the placement of coronary-artery stents. N Engl J Med 1996;334:1084-1089.

3. McFadden EP, Stabile E, Regar E, Cheneau E, Ong AT, Kinnaird T, Suddath WO, Weissman NJ, Torguson R, Kent KM, Pichard AD, Satler LF, Waksman R, Serruys PW. Late thrombosis in drug-eluting coronary stents after discontinuation of antiplatelet therapy. Lancet 2004;364:1519-1521.

4. Valgimigli M, Costa F, Byrne R, Haude M, Baumbach A, Windecker S. Dual antiplatelet therapy duration after coronary stenting in clinical practice: results of an EAPCI survey. Eurolntervention 2015;11:68-74.

5. Mozaffarian D, Benjamin EJ, Go AS, Arnett DK, Blaha MJ, Cushman M, Das SR, de Ferranti S, Despres JP, Fullerton HJ, Howard VJ, Huffman MD, Isasi CR, Jimenez MC, Judd SE, Kissela BM, Lichtman JH, Lisabeth LD, Liu S, Mackey RH, Magid DJ, McGuire DK, Mohler ER III, Moy CS, Muntner P, Mussolino ME, Nasir K, Neumar RW, Nichol G, Palaniappan L, Pandey DK, Reeves MJ, Rodriguez CJ, Rosamond W, Sorlie PD, Stein J, Towfighi A, Turan TN, Virani SS, Woo D, Yeh RW, Turner MB; the American Heart Association Statistics Committee; Stroke Statistics Subcommittee. Heart disease and stroke statistics-2016 update: a report from the American Heart Association. Circulation 2016;133:e38-e360.

6. Bueno $\mathrm{H}$, Fernandez-Aviles F. Use of risk scores in acute coronary syndromes. Heart 2012;98:162-168.

7. Antman EM, Cohen M, Bernink PJ, McCabe CH, Horacek T, Papuchis G, Mautner B, Corbalan R, Radley D, Braunwald E. The TIMI risk score for unstable angina/non-ST elevation MI: A method for prognostication and therapeutic decision making. JAMA 2000;284:835-842.

8. Boersma E, Pieper KS, Steyerberg EW, Wilcox RG, Chang WC, Lee KL, Akkerhuis KM, Harrington RA, Deckers JW, Armstrong PW, Lincoff AM, Califf RM, Topol EJ, Simoons ML. Predictors of outcome in patients with acute coronary syndromes without persistent ST-segment elevation. Results from an international trial of 9461 patients. The PURSUIT Investigators. Circulation 2000;101:2557-2567.

9. Fox KA, Dabbous OH, Goldberg RJ, Pieper KS, Eagle KA, Van de Werf F, Avezum A, Goodman SG, Flather MD, Anderson FA Jr, Granger CB. Prediction of risk of death and myocardial infarction in the six months after presentation with acute coronary syndrome: prospective multinational observational study (GRACE). BMJ 2006;333:1091.

10. Subherwal S, Bach RG, Chen AY, Gage BF, Rao SV, Newby LK, Wang TY, Gibler WB, Ohman EM, Roe MT, Pollack CV Jr, Peterson ED, Alexander KP. Baseline risk of major bleeding in non-ST-segment-elevation myocardial infarction: the CRUSADE (Can Rapid risk stratification of
Unstable angina patients Suppress ADverse outcomes with Early implementation of the ACC/AHA Guidelines) bleeding score. Circulation 2009;119:1873-1882.

11. Mehran R, Pocock SJ, Nikolsky E, Clayton T, Dangas GD, Kirtane AJ, Parise H, Fahy M, Manoukian SV, Feit F, Ohman ME, Witzenbichler B, Guagliumi G, Lansky AJ, Stone GW. A risk score to predict bleeding in patients with acute coronary syndromes. J Am Coll Cardiol 2010;55:2556-2566.

12. Mathews R, Peterson ED, Chen AY, Wang TY, Chin CT, Fonarow GC, Cannon CP, Rumsfeld JS, Roe MT, Alexander KP. In-hospital major bleeding during STelevation and non-ST-elevation myocardial infarction care: derivation and validation of a model from the ACTION Registry(R)-GWTG. Am J Cardiol 2011;107:1136-1143.

13. Costa F, Tijssen JG, Ariotti S, Giatti S, Moscarella E, Guastaroba P, De Palma R, Ando G, Oreto G, Zijlstra F, Valgimigli M. Incremental value of the CRUSADE, ACUITY, and HAS-BLED risk scores for the prediction of hemorrhagic events after coronary stent implantation in patients undergoing long or short duration of dual antiplatelet therapy. J Am Heart Assoc 2015;4:e002524.

14. Mahaffey KW, Yang Q, Pieper KS, Antman EM, White HD, Goodman SG, Cohen M, Kleiman NS, Langer A, Aylward PE, Col JJ, Reist C, Ferguson JJ, Califf RM, Synergy Trial Investigators. Prediction of one-year survival in high-risk patients with acute coronary syndromes: results from the SYNERGY trial. J Gen Intern Med 2008;23:310-316.

15. Yeh RW, Secemsky EA, Kereiakes DJ, Normand SL, Gershlick AH, Cohen DJ, Spertus JA, Steg PG, Cutlip DE, Rinaldi MJ, Camenzind E, Wijns W, Apruzzese PK, Song Y, Massaro JM, Mauri L, DAPT Study Investigators. Development and validation of a prediction rule for benefit and harm of dual antiplatelet therapy beyond 1 year after percutaneous coronary intervention. JAMA 2016;315:1735-1749.

16. Baber U, Mehran R, Giustino G, Cohen DJ, Henry TD, Sartori S, Ariti C, Litherland C, Dangas G, Gibson CM, Krucoff MW, Moliterno DJ, Kirtane AJ, Stone GW, Colombo A, Chieffo A, Kini AS, Witzenbichler B, Weisz G, Steg PG, Pocock S. Coronary thrombosis and major bleeding after PCl with drugeluting stents: risk scores from PARIS. J Am Coll Cardiol 2016;67:2224-2234.

17. Mehran R, Baber U, Steg PG, Ariti C, Weisz G, Witzenbichler B, Henry TD, Kini AS, Stuckey T, Cohen DJ, Berger PB, lakovou I, Dangas G, Waksman R, Antoniucci D, Sartori S, Krucoff MW, Hermiller JB, Shawl F, Gibson CM, Chieffo A, Alu M, Moliterno DJ, Colombo A, Pocock S. Cessation of dual antiplatelet treatment and cardiac events after percutaneous coronary intervention (PARIS): 2 year results from a prospective observational study. Lancet 2013;382:1714-1722.

18. Costa F, van Klaveren D, James S, Heg D, Raber L, Feres F, Pilgrim T, Hong MK, Kim HS, Colombo A, Steg G, Zanchin T, Palmerini T, Wallentin L, Bhatt DL, Stone GW, Windecker S, Steyerberg EW, Valgimigli M. Derivation and validation of the predicting bleeding complications in patients undergoing stent implantation and subsequent dual antiplatelet therapy (PRECISE-DAPT) score: a pooled analysis of individual-patient datasets from clinical trials. Lancet 2017;389:1025-1034.

19. Koskinas KC, Raber L, Zanchin T, Wenaweser P, Stortecky S, Moschovitis A, Khattab AA, Pilgrim T, Blochlinger S, Moro C, Juni P, Meier B, Heg D, Windecker S. Clinical impact of gastrointestinal bleeding in patients undergoing percutaneous coronary interventions. Circ Cardiovasc Interv 2015;8(5):e002053.

20. Wallentin L, Becker RC, Budaj A, Cannon CP, Emanuelsson H, Held C, Horrow J, Husted S, James S, Katus H, Mahaffey KW, Scirica BM, Skene A, Steg PG, Storey RF, Harrington RA, PLATO Investigators. Ticagrelor versus clopidogrel in patients with acute coronary syndromes. N Engl J Med 2009;361:1045-1057.

21. Bertrand ME, Rupprecht HJ, Urban P, Gershlick AH, CLASSICS Investigators. Double-blind study of the safety of clopidogrel with and without a loading dose in combination with aspirin compared with ticlopidine in combination with aspirin after coronary stenting: the clopidogrel aspirin stent international cooperative study (CLASSICS). Circulation 2000;102:624-629.

22. Campo G, Valgimigli M, Gemmati D, Percoco G, Catozzi L, Frangione A, Federici F, Ferrari F, Tebaldi M, Luccarelli S, Parrinello G, Ferrari R. Poor responsiveness to clopidogrel: drug-specific or class-effect mechanism? Evidence from a clopidogrel-to-ticlopidine crossover study. J Am Coll Cardiol 2007;50:1132-1137.

23. Wiviott SD, Braunwald E, McCabe CH, Montalescot G, Ruzyllo W, Gottlieb S, Neumann FJ, Ardissino D, De Servi S, Murphy SA, Riesmeyer J, Weerakkody G, Gibson CM, Antman EM, TRITON-TIMI 38 Investigators. Prasugrel versus clopidogrel in patients with acute coronary syndromes. $N$ Engl J Med 2007;357:2001-2015.

24. Roe MT, Armstrong PW, Fox KA, White HD, Prabhakaran D, Goodman SG, Cornel JH, Bhatt DL, Clemmensen P, Martinez F, Ardissino D, Nicolau JC, Boden WE, Gurbel PA, Ruzyllo W, Dalby AJ, McGuire DK, Leiva-Pons JL, Parkhomenko A, Gottlieb S, Topacio GO, Hamm C, Pavlides G, Goudev AR, Oto A, Tseng CD, Merkely B, Gasparovic V, Corbalan R, Cinteza M, McLendon 
RC, Winters KJ, Brown EB, Lokhnygina Y, Aylward PE, Huber K, Hochman JS, Ohman EM; TRILOGY ACS Investigators TA. Prasugrel versus clopidogrel for acute coronary syndromes without revascularization. N Engl J Med 2012;367:1297-1309.

25. Montalescot G, Bolognese L, Dudek D, Goldstein P, Hamm C, Tanguay JF, ten Berg JM, Miller DL, Costigan TM, Goedicke J, Silvain J, Angioli P, Legutko J, Niethammer M, Motovska Z, Jakubowski JA, Cayla G, Visconti LO, Vicaut E, Widimsky P. Pretreatment with prasugrel in non-ST-segment elevation acute coronary syndromes. N Engl J Med 2013;369:999-1010.

26. Mauri L, Kereiakes DJ, Yeh RW, Driscoll-Shempp P, Cutlip DE, Steg PG, Normand SL, Braunwald E, Wiviott SD, Cohen DJ, Holmes DR Jr, Krucoff MW, Hermiller J, Dauerman HL, Simon DI, Kandzari DE, Garratt KN, Lee DP, Pow TK, Ver Lee P, Rinaldi MJ, Massaro JM, DAPT Study Investigators. Twelve or 30 months of dual antiplatelet therapy after drug-eluting stents. N Engl J Med 2014;371:2155-2166.

27. Garratt KN, Weaver WD, Jenkins RG, Pow TK, Mauri L, Kereiakes DJ, Winters $\mathrm{KJ}$, Christen T, Allocco DJ, Lee DP. Prasugrel plus aspirin beyond 12 months is associated with improved outcomes after TAXUS Liberté paclitaxel-eluting coronary stent placement. Circulation 2015;131:62-73.

28. Montalescot G, van 't Hof AW, Lapostolle F, Silvain J, Lassen JF, Bolognese L, Cantor WJ, Cequier A, Chettibi M, Goodman SG, Hammett C], Huber K, Janzon M, Merkely B, Storey RF, Zeymer U, Stibbe O, Ecollan P, Heutz WM, Swahn E, Collet JP, Willems FF, Baradat C, Licour M, Tsatsaris A, Vicaut E, Hamm CW. Prehospital ticagrelor in ST-segment elevation myocardial infarction. N EnglJ Med 2014;371:1016-1027.

29. Bonaca MP, Bhatt DL, Cohen M, Steg PG, Storey RF, Jensen EC, Magnani G, Bansilal S, Fish MP, Im K, Bengtsson O, Oude Ophuis T, Budaj A, Theroux P, Ruda M, Hamm C, Goto S, Spinar J, Nicolau JC, Kiss RG, Murphy SA, Wiviott SD, Held P, Braunwald E, Sabatine MS, PEGASUS-TIMI 54 Steering Committee and Investigators. Long-term use of ticagrelor in patients with prior myocardial infarction. N Engl J Med 2015;372:1791-1800.

30. Armstrong PW, Gershlick AH, Goldstein P, Wilcox R, Danays T, Lambert $Y$, Sulimov V, Rosell Ortiz F, Ostojic M, Welsh RC, Carvalho AC, Nanas J, Arntz HR, Halvorsen S, Huber K, Grajek S, Fresco C, Bluhmki E, Regelin A, Vandenberghe K, Bogaerts K, Van de Werf F; STREAM Investigative Team. Fibrinolysis or primary $\mathrm{PCl}$ in ST-segment elevation myocardial infarction. $\mathrm{N}$ EnglJ Med 2013;368:1379-1387.

31. Sabatine MS, Cannon CP, Gibson CM, Lopez-Sendon JL, Montalescot G, Theroux P, Claeys MJ, Cools F, Hill KA, Skene AM, McCabe CH, Braunwald E, CLARITY-TIMI 28 Investigators. Addition of clopidogrel to aspirin and fibrinolytic therapy for myocardial infarction with ST-segment elevation. N Engl J Med 2005;352:1179-1189.

32. Chen ZM, Jiang LX, Chen YP, Xie JX, Pan HC, Peto R, Collins R, Liu LS. Addition of clopidogrel to aspirin in 45,852 patients with acute myocardial infarction: randomised placebo-controlled trial. Lancet 2005;366:1607-1621.

33. Montalescot G, Wiviott SD, Braunwald E, Murphy SA, Gibson CM, McCabe $\mathrm{CH}$, Antman EM, TRITON-TIMI 38 Investigators. Prasugrel compared with clopidogrel in patients undergoing percutaneous coronary intervention for STelevation myocardial infarction (TRITON-TIMI 38): double-blind, randomised controlled trial. Lancet 2009;373:723-731.

34. Roffi M, Patrono C, Collet JP, Mueller C, Valgimigli M, Andreotti F, Bax JJ, Borger MA, Brotons C, Chew DP, Gencer B, Hasenfuss G, Kjeldsen K, Lancellotti P, Landmesser U, Mehilli J, Mukherjee D, Storey RF, Windecker S, Baumgartner H, Gaemperli O, Achenbach S, Agewall S, Badimon L, Baigent C, Bueno H, Bugiardini R, Carerj S, Casselman F, Cuisset T, Erol C, Fitzsimons D, Halle M, Hamm C, Hildick-Smith D, Huber K, lliodromitis E, James S, Lewis BS, Lip GY, Piepoli MF, Richter D, Rosemann T, Sechtem U, Steg PG, Vrints C, Luis Zamorano J. 2015 ESC Guidelines for the management of acute coronary syndromes in patients presenting without persistent ST-segment elevation: Task Force for the Management of Acute Coronary Syndromes in Patients Presenting without Persistent ST-Segment Elevation of the European Society of Cardiology (ESC). Eur Heart / 2016;37:267-315.

35. Valgimigli M. Pretreatment with P2Y12 inhibitors in non-ST-segment-elevation acute coronary syndrome is clinically justified. Circulation 2014;130:1891-1903; discussion 1903.

36. Collet JP, Silvain J, Bellemain-Appaix A, Montalescot G. Pretreatment with P2Y12 inhibitors in non-ST-Segment-elevation acute coronary syndrome: an outdated and harmful strategy. Circulation 2014;130:1904-1914; discussion 1914.

37. Rollini F, Franchi F, Angiolillo DJ. Switching P2Y12-receptor inhibitors in patients with coronary artery disease. Nat Rev Cardiol 2016;13:11-27.

38. Bellemain-Appaix A, O'Connor SA, Silvain J, Cucherat M, Beygui F, Barthelemy O, Collet JP, Jacq L, Bernasconi F, Montalescot G; ACTION Group. Association of clopidogrel pretreatment with mortality, cardiovascular events, and major bleeding among patients undergoing percutaneous coronary intervention: a systematic review and meta-analysis. JAMA 2012;308:2507-2516.
39. Steinhubl SR, Berger PB, Mann JT III, Fry ET, DeLago A, Wilmer C, Topol EJ. Early and sustained dual oral antiplatelet therapy following percutaneous coronary intervention: a randomized controlled trial. JAMA 2002;288 2411-2420.

40. Yusuf S, Zhao F, Mehta SR, Chrolavicius S, Tognoni G, Fox KK, Clopidogrel in Unstable Angina to Prevent Recurrent Events Trial Investigators. Effects of clopidogrel in addition to aspirin in patients with acute coronary syndromes without ST-segment elevation. N Engl J Med 2001;345:494-502.

41. Steg PG, Huber K, Andreotti F, Arnesen H, Atar D, Badimon L, Bassand JP, De Caterina R, Eikelboom JA, Gulba D, Hamon M, Helft G, Fox KA, Kristensen SD, Rao SV, Verheugt FW, Widimsky P, Zeymer U, Collet JP. Bleeding in acute coronary syndromes and percutaneous coronary interventions: position paper by the Working Group on Thrombosis of the European Society of Cardiology. Eur Heart J 2011;32:1854-1864.

42. Valgimigli M, Costa F, Lokhnygina $Y$, Clare R, Wallentin L, Moliterno D, Armstrong P, White H, Held C, Aylward P, Van de Werf F, Harrington R, Mahaffey K, Tricoci P. Trade-off of myocardial infarction vs. bleeding types on mortality after acute coronary syndrome: lessons from the Thrombin Receptor Antagonist for Clinical Event Reduction in Acute Coronary Syndrome (TRACER) randomized trial. Eur Heart J 2017:38:804-810.

43. Valgimigli M, Gagnor A, Calabro P, Frigoli E, Leonardi S, Zaro T, Rubartelli P, Briguori C, Ando G, Repetto A, Limbruno U, Cortese B, Sganzerla P, Lupi A, Galli M, Colangelo S, lerna S, Ausiello A, Presbitero P, Sardella G, Varbella F, Esposito G, Santarelli A, Tresoldi S, Nazzaro M, Zingarelli A, de Cesare N, Rigattieri S, Tosi P, Palmieri C, Brugaletta S, Rao SV, Heg D, Rothenbuhler M, Vranckx P, Juni P; MATRIX Investigators. Radial versus femoral access in patients with acute coronary syndromes undergoing invasive management: a randomised multicentre trial. Lancet 2015;385:2465-2476.

44. Ferrante G, Rao SV, Juni P, Da Costa BR, Reimers B, Condorelli G, Anzuini A, Jolly SS, Bertrand OF, Krucoff MW, Windecker S, Valgimigli M. Radial versus femoral access for coronary interventions across the entire spectrum of patients with coronary artery disease: a meta-analysis of randomized trials. JACC Cardiovasc Interv 2016;9:1419-1434.

45. Antithrombotic Trialists Collaboration. Collaborative meta-analysis of randomised trials of antiplatelet therapy for prevention of death, myocardial infarction, and stroke in high risk patients. BMJ 2002;324:71-86.

46. Lorenz RL, Schacky CV, Weber M, Meister W, Kotzur J, Reichardt B, Theisen K, Weber PC. Improved aortocoronary bypass patency by low-dose aspirin $(100 \mathrm{mg}$ daily). Effects on platelet aggregation and thromboxane formation. Lancet 1984;1:1261-1264.

47. Peters RJ, Mehta SR, Fox KA, Zhao F, Lewis BS, Kopecky SL, Diaz R, Commerford PJ, Valentin V, Yusuf S. Effects of aspirin dose when used alone or in combination with clopidogrel in patients with acute coronary syndromes: observations from the Clopidogrel in Unstable angina to prevent Recurrent Events (CURE) study. Circulation 2003;108:1682-1687.

48. Serebruany VL, Steinhubl SR, Berger PB, Malinin AI, Baggish JS, Bhatt DL, Topol EJ. Analysis of risk of bleeding complications after different doses of aspirin in 192,036 patients enrolled in 31 randomized controlled trials. Am J Cardiol 2005;95:1218-1222.

49. Jolly SS, Pogue J, Haladyn K, Peters RJ, Fox KA, Avezum A, Gersh BJ, Rupprecht HJ, Yusuf S, Mehta SR. Effects of aspirin dose on ischaemic events and bleeding after percutaneous coronary intervention: insights from the PCI-CURE study. Eur Heart J 2009;30:900-907.

50. Xian Y, Wang TY, McCoy LA, Effron MB, Henry TD, Bach RG, Zettler ME, Baker BA, Fonarow GC, Peterson ED. Association of discharge aspirin dose with outcomes after acute myocardial infarction: insights from the Treatment with ADP Receptor Inhibitors: Longitudinal Assessment of Treatment Patterns and Events after Acute Coronary Syndrome (TRANSLATE-ACS) Study. Circulation 2015;132:174-181.

51. Steinhubl SR, Bhatt DL, Brennan DM, Montalescot G, Hankey GJ, Eikelboom JW, Berger PB, Topol EJ, CHARISMA Investigators. Aspirin to prevent cardiovascular disease: the association of aspirin dose and clopidogrel with thrombosis and bleeding. Ann Intern Med 2009;150:379-386.

52. Mehta SR, Tanguay JF, Eikelboom JW, Jolly SS, Joyner CD, Granger CB, Faxon DP, Rupprecht HJ, Budaj A, Avezum A, Widimsky P, Steg PG, Bassand JP, Montalescot G, Macaya C, Di Pasquale G, Niemela K, Ajani AE, White HD, Chrolavicius S, Gao P, Fox KA, Yusuf S. Double-dose versus standarddose clopidogrel and high-dose versus low-dose aspirin in individuals undergoing percutaneous coronary intervention for acute coronary syndromes (CURRENT-OASIS 7): a randomised factorial trial. Lancet 2010;376: 1233-1243.

53. Montalescot G, Drobinski G, Maclouf J, Maillet F, Salloum J, Ankri A, Kazatchkine M, Eugene L, Thomas D, Grosgogeat Y. Evaluation of thromboxane production and complement activation during myocardial ischemia in patients with angina pectoris. Circulation 1991;84:2054-2062. 
54. Patrono C, Ciabattoni G, Patrignani P, Pugliese F, Filabozzi P, Catella F, Davi G, Forni L. Clinical pharmacology of platelet cyclooxygenase inhibition. Circulation 1985;72:1177-1184.

55. Mahaffey KW, Wojdyla DM, Carroll K, Becker RC, Storey RF, Angiolillo DJ, Held C, Cannon CP, James S, Pieper KS, Horrow J, Harrington RA, Wallentin L. Ticagrelor compared with clopidogrel by geographic region in the Platelet Inhibition and Patient Outcomes (PLATO) trial. Circulation 2011;124:544-554.

56. Aradi D, Kirtane A, Bonello L, Gurbel PA, Tantry US, Huber K, Freynhofer MK, ten Berg J, Janssen P, Angiolillo DJ, Siller-Matula JM, Marcucci R, Patti G, Mangiacapra F, Valgimigli M, Morel O, Palmerini T, Price MJ, Cuisset T, Kastrati A, Stone GW, Sibbing D. Bleeding and stent thrombosis on P2Y12-inhibitors: collaborative analysis on the role of platelet reactivity for risk stratification after percutaneous coronary intervention. Eur Heart J 2015;36:1762-1771.

57. Bonello L, Tantry US, Marcucci R, Blindt R, Angiolillo DJ, Becker R, Bhatt DL, Cattaneo M, Collet JP, Cuisset T, Gachet C, Montalescot G, Jennings LK, Kereiakes D, Sibbing D, Trenk D, Van Werkum JW, Paganelli F, Price MJ, Waksman R, Gurbel PA, the Working Group on High On-Treatment Platelet Reactivity. Consensus and future directions on the definition of high ontreatment platelet reactivity to adenosine diphosphate. J Am Coll Cardiol 2010;56:919-933.

58. Collet JP, Cuisset T, Range G, Cayla G, Elhadad S, Pouillot C, Henry P, Motreff P, Carrie D, Boueri Z, Belle L, Van Belle E, Rousseau H, Aubry P, Monsegu J, Sabouret P, O'Connor SA, Abtan J, Kerneis M, Saint-Etienne C, Barthelemy O, Beygui F, Silvain J, Vicaut E, Montalescot G. Bedside monitoring to adjust antiplatelet therapy for coronary stenting. N Engl J Med 2012;367:2100-2109.

59. Trenk D, Stone GW, Gawaz M, Kastrati A, Angiolillo DJ, Muller U, Richardt G, Jakubowski JA, Neumann FJ. A randomized trial of prasugrel versus clopidogrel in patients with high platelet reactivity on clopidogrel after elective percutaneous coronary intervention with implantation of drug-eluting stents: results of the TRIGGER-PCI (Testing Platelet Reactivity In Patients Undergoing Elective Stent Placement on Clopidogrel to Guide Alternative Therapy With Prasugrel) study. J Am Coll Cardiol 2012;59:2159-2164.

60. Price MJ, Berger PB, Teirstein PS, Tanguay JF, Angiolillo DJ, Spriggs D, Puri S, Robbins M, Garratt KN, Bertrand OF, Stillabower ME, Aragon JR, Kandzari DE, Stinis CT, Lee MS, Manoukian SV, Cannon CP, Schork NJ, Topol EJ, GRAVITAS Investigators. Standard- vs high-dose clopidogrel based on platelet function testing after percutaneous coronary intervention: the GRAVITAS randomized trial. JAMA for 2011;305:1097-1105.

61. Montalescot G, Vicaut E, Collet JP. Bedside monitoring of antiplatelet therapy for coronary stenting. N Engl J Med 2013;368:871-872.

62. Lim GB. Antiplatelet therapy. ARCTIC leaves platelet testing out in the cold. Nat Rev Cardiol 2013;10:2

63. De Miguel Castro A, Nieto AD, Perez de Prado A. Letter by De Miguel Castro et al regarding article, "Platelet reactivity and cardiovascular outcomes after percutaneous coronary intervention: a time-dependent analysis of the gauging responsiveness with a VerifyNow P2Y12 assay: impact on Thrombosis and Safety (GRAVITAS) trial". Circulation 2012;125:e570; author reply e571-572.

64. Cayla G, Cuisset T, Silvain J, Leclercq F, Manzo-Silberman S, Saint-Etienne C, Delarche N, Bellemain-Appaix A, Range G, El Mahmoud R, Carrie D, Belle L, Souteyrand G, Aubry P, Sabouret P, du Fretay XH, Beygui F, Bonnet JL, Lattuca B, Pouillot C, Varenne O, Boueri Z, Van Belle E, Henry P, Motreff P, Elhadad S, Salem JE, Abtan J, Rousseau H, Collet JP, Vicaut E, Montalescot G. Platelet function monitoring to adjust antiplatelet therapy in elderly patients stented for an acute coronary syndrome (ANTARCTIC): an open-label, blinded-endpoint, randomised controlled superiority trial. Lancet 2016;388:2015-2022.

65. Mega JL, Simon T, Collet JP, Anderson JL, Antman EM, Bliden K, Cannon CP, Danchin N, Giusti B, Gurbel P, Horne BD, Hulot JS, Kastrati A, Montalescot G, Neumann FJ, Shen L, Sibbing D, Steg PG, Trenk D, Wiviott SD, Sabatine MS. Reduced-function CYP2C19 genotype and risk of adverse clinical outcomes among patients treated with clopidogrel predominantly for PCl: a meta-analysis. JAMA 2010;304:1821-1830.

66. Collet JP, Kerneis M, Hulot JS, O'Connor SA, Silvain J, Mansencal N, Brugier D, Abtan J, Barthelemy O, Vignalou JB, Payot L, Rousseau H, Vicaut E, Montalescot G. Point-of-care genetic profiling and/or platelet function testing in acute coronary syndrome. Thromb Haemost 2016;115:382-391.

67. Roberts JD, Wells GA, Le May MR, Labinaz M, Glover C, Froeschl M, Dick A, Marquis JF, O'Brien E, Goncalves S, Druce I, Stewart A, Gollob MH, So DY. Point-of-care genetic testing for personalisation of antiplatelet treatment (RAPID GENE): a prospective, randomised, proof-of-concept trial. Lancet 2012;379:1705-1711.

68. Shuldiner AR, O'Connell JR, Bliden KP, Gandhi A, Ryan K, Horenstein RB, Damcott CM, Pakyz R, Tantry US, Gibson Q, Pollin TI, Post W, Parsa A, Mitchell BD, Faraday N, Herzog W, Gurbel PA. Association of cytochrome P450 2C19 genotype with the antiplatelet effect and clinical efficacy of clopidogrel therapy. JAMA 2009;302:849-857.
69. Hochholzer W, Trenk D, Fromm MF, Valina CM, Stratz C, Bestehorn HP, Buttner HJ, Neumann FJ. Impact of cytochrome P4502C19 loss-of-function polymorphism and of major demographic characteristics on residual platelet function after loading and maintenance treatment with clopidogrel in patients undergoing elective coronary stent placement. I Am Coll Cardiol 2010;55:2427-2434.

70. Agewall S, Cattaneo M, Collet JP, Andreotti F, Lip GY, Verheugt FW, Huber K, Grove EL, Morais J, Husted S, Wassmann S, Rosano G, Atar D, Pathak A, Kjeldsen K, Storey RF. Expert position paper on the use of proton pump inhibitors in patients with cardiovascular disease and antithrombotic therapy. Eur Heart J 2013;34:1708-1713, 1713a-1713b.

71. Lai KC, Lam SK, Chu KM, Wong BC, Hui WM, Hu WH, Lau GK, Wong WM, Yuen MF, Chan AO, Lai CL, Wong J. Lansoprazole for the prevention of recurrences of ulcer complications from long-term low-dose aspirin use. N Engl J Med 2002;346:2033-2038.

72. Taha AS, McCloskey C, Prasad R, Bezlyak V. Famotidine for the prevention of peptic ulcers and oesophagitis in patients taking low-dose aspirin (FAMOUS): a phase III, randomised, double-blind, placebo-controlled trial. Lancet 2009;374:119-125.

73. Small DS, Farid NA, Payne CD, Weerakkody GJ, Li YG, Brandt JT, Salazar DE, Winters KJ. Effects of the proton pump inhibitor lansoprazole on the pharmacokinetics and pharmacodynamics of prasugrel and clopidogrel. J Clin Pharmacol 2008;48:475-484

74. Sibbing D, Morath T, Stegherr J, Braun S, Vogt W, Hadamitzky M, Schomig A, Kastrati A, von Beckerath N. Impact of proton pump inhibitors on the antiplatelet effects of clopidogrel. Thromb Haemost 2009;101:714-719.

75. Gilard M, Arnaud B, Cornily JC, Le Gal G, Lacut K, Le Calvez G, Mansourati J, Mottier D, Abgrall JF, Boschat J. Influence of omeprazole on the antiplatelet action of clopidogrel associated with aspirin: the randomized, double-blind OCLA (Omeprazole CLopidogrel Aspirin) study. I Am Coll Cardiol 2008;51:256-260.

76. O'Donoghue ML, Braunwald E, Antman EM, Murphy SA, Bates ER, Rozenman Y, Michelson AD, Hautvast RW, Ver Lee PN, Close SL, Shen L, Mega JL, Sabatine MS, Wiviott SD. Pharmacodynamic effect and clinical efficacy of clopidogrel and prasugrel with or without a proton-pump inhibitor: an analysis of two randomised trials. Lancet 2009;374:989-997.

77. Norgard NB, Mathews KD, Wall GC. Drug-drug interaction between clopidogrel and the proton pump inhibitors. Ann Pharmacother 2009;43:1266-1274.

78. Shah NH, LePendu P, Bauer-Mehren A, Ghebremariam YT, lyer SV, Marcus J, Nead KT, Cooke JP, Leeper NJ. Proton pump inhibitor usage and the risk of myocardial infarction in the general population. PLoS One 2015;10:e0124653.

79. Bhatt DL, Cryer BL, Contant CF, Cohen M, Lanas A, Schnitzer TJ, Shook TL, Lapuerta P, Goldsmith MA, Laine L, Scirica BM, Murphy SA, Cannon CP. Clopidogrel with or without omeprazole in coronary artery disease. N Engl J Med 2010;363:1909-1917.

80. Gargiulo G, Costa F, Ariotti S, Biscaglia S, Campo G, Esposito G, Leonardi S, Vranckx P, Windecker S, Valgimigli M. Impact of proton pump inhibitors on clinical outcomes in patients treated with a 6- or 24-month dual-antiplatelet therapy duration: Insights from the PROlonging Dual-antiplatelet treatment after Grading stent-induced Intimal hyperplasia studY trial. Am Heart J 2016;174:95-102

81. Goodman SG, Clare R, Pieper KS, Nicolau JC, Storey RF, Cantor WJ, Mahaffey KW, Angiolillo DJ, Husted S, Cannon CP, James SK, Kilhamn J, Steg PG, Harrington RA, Wallentin L; Platelet Inhibition and Patient Outcomes Trial Investigators. Association of proton pump inhibitor use on cardiovascular outcomes with clopidogrel and ticagrelor: insights from the platelet inhibition and patient outcomes trial. Circulation 2012;125:978-986.

82. Becker RC, Bassand JP, Budaj A, Wojdyla DM, James SK, Cornel JH, French J, Held C, Horrow J, Husted S, Lopez-Sendon J, Lassila R, Mahaffey KW, Storey RF, Harrington RA, Wallentin L. Bleeding complications with the P2Y12 receptor antagonists clopidogrel and ticagrelor in the PLATelet inhibition and patient Outcomes (PLATO) trial. Eur Heart J 2011;32:2933-2944.

83. de la Coba Ortiz C, Arguelles Arias F, Martin de Argila de Prados C, Judez Gutierrez J, Linares Rodriguez A, Ortega Alonso A, Rodriguez de Santiago E, Rodriguez-Tellez M, Vera Mendoza MI, Aguilera Castro L, Alvarez Sanchez A, Andrade Bellido RJ, Bao Perez F, Castro Fernandez M, Giganto Tome F. Proton-pump inhibitors adverse effects: a review of the evidence and position statement by the Sociedad Espanola de Patologia Digestiva. Rev Esp Enferm Dig 2016; 108:207-224.

84. Kerneis M, Silvain J, Abtan J, Cayla G, O'Connor SA, Barthelemy O, Vignalou JB, Beygui F, Brugier D, Martin R, Collet JP, Montalescot G. Switching acute coronary syndrome patients from prasugrel to clopidogrel. JACC Cardiovasc Interv 2013;6:158-165.

85. Stone GW, Witzenbichler B, Weisz G, Rinaldi MJ, Neumann FJ, Metzger DC, Henry TD, Cox DA, Duffy PL, Mazzaferri E, Gurbel PA, Xu K, Parise H, Kirtane 
AJ, Brodie BR, Mehran R, Stuckey TD, ADAPT-DES Investigators. Platelet reactivity and clinical outcomes after coronary artery implantation of drug-eluting stents (ADAPT-DES): a prospective multicentre registry study. Lancet 2013;382:614-623.

86. Moukarbel GV, Bhatt DL. Antiplatelet therapy and proton pump inhibition: clinician update. Circulation 2012;125:375-380.

87. Fortuna LA, Pawloski PA, Parker ED, Trower NK, Kottke TE. Proton pump inhibitor use by aspirin-treated coronary artery disease patients is not associated with increased risk of cardiovascular events. Eur Heart J Cardiovasc Pharmacother 2016;2:13-19.

88. Cannon CP, Harrington RA, James S, Ardissino D, Becker RC, Emanuelsson H, Husted S, Katus H, Keltai M, Khurmi NS, Kontny F, Lewis BS, Steg PG, Storey RF, Wojdyla D, Wallentin L; PLATelet inhibition and patient Outcomes Investigators. Comparison of ticagrelor with clopidogrel in patients with a planned invasive strategy for acute coronary syndromes (PLATO): a randomised double-blind study. Lancet 2010;375:283-293.

89. Bagai A, Peterson ED, Honeycutt E, Effron MB, Cohen DJ, Goodman SG, Anstrom KJ, Gupta A, Messenger JC, Wang TY. In-hospital switching between adenosine diphosphate receptor inhibitors in patients with acute myocardial infarction treated with percutaneous coronary intervention: Insights into contemporary practice from the TRANSLATE-ACS study. Eur Heart I Acute Cardiovasc Care 2015;4:499-508.

90. Clemmensen P, Grieco N, Ince H, Danchin N, Goedicke J, Ramos Y, Schmitt J, Goldstein P. MULTInational non-interventional study of patients with STsegment elevation myocardial infarction treated with PRimary Angioplasty and Concomitant use of upstream antiplatelet therapy with prasugrel or clopidogrel-the European MULTIPRAC Registry. Eur Heart J Acute Cardiovasc Care 2015;4:220-229.

91. Alexopoulos D, Xanthopoulou I, Deftereos S, Sitafidis G, Kanakakis I, Hamilos M, Angelidis C, Petousis S, Stakos D, Parissis H, Vavouranakis M, Davlouros P, Goudevenos J, Stefanadis C. In-hospital switching of oral P2Y12 inhibitor treatment in patients with acute coronary syndrome undergoing percutaneous coronary intervention: prevalence, predictors and short-term outcome. Am Heart J 2014; 167:68-76 e2

92. Franchi F, Faz GT, Rollini F, Park Y, Cho JR, Thano E, Hu J, Kureti M, Aggarwal N, Durairaj A, Been L, Zenni MM, Guzman LA, Suryadevara S, Antoun P, Bass TA, Angiolillo DJ. Pharmacodynamic effects of switching from prasugrel to ticagrelor: results of the prospective, randomized SWAP-3 study. JACC Cardiovasc Interv 2016;9:1089-1098.

93. Angiolillo DJ, Curzen N, Gurbel P, Vaitkus P, Lipkin F, Li W, Jakubowski JA, Zettler M, Effron MB, Trenk D. Pharmacodynamic evaluation of switching from ticagrelor to prasugrel in patients with stable coronary artery disease: results of the SWAP-2 study (Switching Anti Platelet-2). I Am Coll Cardiol 2014;63:1500-1509.

94. Angiolillo DJ, Saucedo JF, Deraad R, Frelinger AL, Gurbel PA, Costigan TM, Jakubowski JA, Ojeh CK, Effron MB, SWAP Investigators. Increased platelet inhibition after switching from maintenance clopidogrel to prasugrel in patients with acute coronary syndromes: results of the SWAP (SWitching Anti Platelet) study. J Am Coll Cardiol 2010;56:1017-1023.

95. Bhatt DL, Fox KA, Hacke W, Berger PB, Black HR, Boden WE, Cacoub P, Cohen EA, Creager MA, Easton JD, Flather MD, Haffner SM, Hamm CW, Hankey GJ, Johnston SC, Mak KH, Mas JL, Montalescot G, Pearson TA, Steg PG, Steinhubl SR, Weber MA, Brennan DM, Fabry-Ribaudo L, Booth J, Topol EJ, CHARISMA Investigators. Clopidogrel and aspirin versus aspirin alone for the prevention of atherothrombotic events. N Engl J Med 2006;354:1706-1717.

96. Bertrand ME, Legrand V, Boland J, Fleck E, Bonnier J, Emmanuelson H, Vrolix M, Missault L, Chierchia S, Casaccia M, Niccoli L, Oto A, White C, WebbPeploe M, Van Belle E, McFadden EP. Randomized multicenter comparison of conventional anticoagulation versus antiplatelet therapy in unplanned and elective coronary stenting. Circulation 1998;98:1597-1603.

97. Urban P, Macaya C, Rupprecht HJ, Kiemeneij F, Emanuelsson H, Fontanelli A, Pieper M, Wesseling T, Sagnard L. Randomized evaluation of anticoagulation versus antiplatelet therapy after coronary stent implantation in high-risk patients: the multicenter aspirin and ticlopidine trial after intracoronary stenting (MATTIS). Circulation 1998;98:2126-2132.

98. Yeh RW, Kereiakes DJ, Steg PG, Windecker S, Rinaldi MJ, Gershlick AH, Cutlip DE, Cohen DJ, Tanguay JF, Jacobs A, Wiviott SD, Massaro JM, lancu AC, Mauri L, DAPT Study Investigators. Benefits and risks of extended duration dual antiplatelet therapy after $\mathrm{PCl}$ in patients with and without acute myocardial infarction. J Am Coll Cardiol 2015;65:2211-2221.

99. Costa F, Vranckx P, Leonardi S, Moscarella E, Ando G, Calabro P, Oreto G, Zijlstra F, Valgimigli M. Impact of clinical presentation on ischaemic and bleeding outcomes in patients receiving 6- or 24-month duration of dual-antiplatelet therapy after stent implantation: a pre-specified analysis from the PRODIGY
(Prolonging Dual-Antiplatelet Treatment After Grading Stent-Induced Intimal Hyperplasia) trial. Eur Heart J 2015;36:1242-1251.

100. Gwon HC, Hahn JY, Park KW, Song YB, Chae IH, Lim DS, Han KR, Choi JH, Choi SH, Kang HJ, Koo BK, Ahn T, Yoon JH, Jeong MH, Hong TJ, Chung WY, Choi YJ, Hur SH, Kwon HM, Jeon DW, Kim BO, Park SH, Lee NH, Jeon HK, Jang Y, Kim HS. Six-month versus 12-month dual antiplatelet therapy after implantation of drug-eluting stents: the Efficacy of Xience/Promus Versus Cypher to Reduce Late Loss After Stenting (EXCELLENT) randomized, multicenter study. Circulation 2012;125:505-513.

101. Valgimigli M, Campo G, Monti M, Vranckx P, Percoco G, Tumscitz C, Castriota F, Colombo F, Tebaldi M, Fuca G, Kubbajeh M, Cangiano E, Minarelli M, Scalone A, Cavazza C, Frangione A, Borghesi M, Marchesini J, Parrinello G, Ferrari R. Short- versus long-term duration of dual-antiplatelet therapy after coronary stenting: a randomized multicenter trial. Circulation 2012;125:2015-2026.

102. Gilard M, Barragan P, Noryani AA, Noor HA, Majwal T, Hovasse T, Castellant P, Schneeberger M, Maillard L, Bressolette E, Wojcik J, Delarche N, Blanchard D, Jouve B, Ormezzano O, Paganelli F, Levy G, Sainsous J, Carrie D, Furber A, Berland J, Darremont O, Le Breton H, Lyuycx-Bore A, Gommeaux A, Cassat C, Kermarrec A, Cazaux P, Druelles P, Dauphin R, Armengaud J, Dupouy P, Champagnac D, Ohlmann P, Endresen K, Benamer H, Kiss RG, Ungi I, Boschat J, Morice MC. 6- versus 24-month dual antiplatelet therapy after implantation of drug-eluting stents in patients nonresistant to aspirin: the randomized, multicenter ITALIC trial. J Am Coll Cardiol 2015;65:777-786.

103. Colombo A, Chieffo A, Frasheri A, Garbo R, Masotti-Centol M, Salvatella N, Oteo Dominguez JF, Steffanon L, Tarantini G, Presbitero P, Menozzi A, Pucci E, Mauri J, Cesana BM, Giustino G, Sardella G. Second-generation drug-eluting stent implantation followed by 6- versus 12-month dual antiplatelet therapy: the SECURITY randomized clinical trial. J Am Coll Cardiol 2014;64:2086-2097.

104. Schulz-Schupke S, Byrne RA, Ten Berg JM, Neumann FJ, Han Y, Adriaenssens T, Tolg R, Seyfarth M, Maeng M, Zrenner B, Jacobshagen C, Mudra H, von Hodenberg E, Wohrle J, Angiolillo DJ, von Merzljak B, Rifatov N, Kufner S, Morath T, Feuchtenberger A, Ibrahim T, Janssen PW, Valina C, Li Y, Desmet W, Abdel-Wahab M, Tiroch K, Hengstenberg C, Bernlochner I, Fischer M, Schunkert H, Laugwitz KL, Schomig A, Mehilli J, Kastrati A. ISAR-SAFE: a randomized, double-blind, placebo-controlled trial of 6 vs. 12 months of clopidogrel therapy after drug-eluting stenting. Eur Heart J 2015;36:1252-1263.

105. Kim BK, Hong MK, Shin DH, Nam CM, Kim JS, Ko YG, Choi D, Kang TS, Park BE, Kang WC, Lee SH, Yoon JH, Hong BK, Kwon HM, Jang Y. A new strategy for discontinuation of dual antiplatelet therapy: the RESET Trial (REal Safety and Efficacy of 3-month dual antiplatelet Therapy following Endeavor zotarolimus-eluting stent implantation). J Am Coll Cardiol 2012;60:1340-1348.

106. Feres F, Costa RA, Abizaid A, Leon MB, Marin-Neto JA, Botelho RV, King SB III, Negoita M, Liu M, de Paula JE, Mangione JA, Meireles GX, Castello HJ Jr, Nicolela EL Jr, Perin MA, Devito FS, Labrunie A, Salvadori D Jr, Gusmao M, Staico R, Costa JR Jr, de Castro JP, Abizaid AS, Bhatt DL, OPTIMIZE Trial investigators. Three vs twelve months of dual antiplatelet therapy after zotarolimus-eluting stents: the OPTIMIZE randomized trial. JAMA 2013;310:2510-2522.

107. Palmerini T, Benedetto U, Bacchi-Reggiani L, Della Riva D, Biondi-Zoccai $G$, Feres F, Abizaid A, Hong MK, Kim BK, Jang Y, Kim HS, Park KW, Genereux P, Bhatt DL, Orlandi C, De Servi S, Petrou M, Rapezzi C, Stone GW. Mortality in patients treated with extended duration dual antiplatelet therapy after drugeluting stent implantation: a pairwise and Bayesian network meta-analysis of randomised trials. Lancet 2015;385:2371-2382.

108. Navarese EP, Andreotti F, Schulze V, Kolodziejczak M, Buffon A, Brouwer M, Costa F, Kowalewski M, Parati G, Lip GY, Kelm M, Valgimigli M. Optimal duration of dual antiplatelet therapy after percutaneous coronary intervention with drug eluting stents: meta-analysis of randomised controlled trials. BM 2015;350:h1618.

109. Giustino G, Baber U, Sartori S, Mehran R, Mastoris I, Kini AS, Sharma SK, Pocock SJ, Dangas GD. Duration of dual antiplatelet therapy after drug-eluting stent implantation: a systematic review and meta-analysis of randomized controlled trials. J Am Coll Cardiol 2015;65:1298-1310.

110. Mauri L, Yeh RW, Kereiakes DJ. Duration of dual antiplatelet therapy after drug-eluting stents. N Engl J Med 2015;372:1373-1374.

111. Lee CW, Ahn JM, Park DW, Kang SJ, Lee SW, Kim YH, Park SW, Han S, Lee SG, Seong IW, Rha SW, Jeong MH, Lim DS, Yoon JH, Hur SH, Choi YS, Yang JY, Lee NH, Kim HS, Lee BK, Kim KS, Lee SU, Chae JK, Cheong SS, Suh IW, Park HS, Nah DY, Jeon DS, Seung KB, Lee K, Jang JS, Park SJ. Optimal duration of dual antiplatelet therapy after drug-eluting stent implantation: a randomized, controlled trial. Circulation 2014;129:304-312.

112. Collet JP, Silvain J, Barthelemy O, Range G, Cayla G, Van Belle E, Cuisset T, Elhadad S, Schiele F, Lhoest N, Ohlmann P, Carrie D, Rousseau H, Aubry P, Monsegu J, Sabouret P, O'Connor SA, Abtan J, Kerneis M, Saint-Etienne C, Beygui F, Vicaut E, Montalescot G. Dual-antiplatelet treatment beyond 1 year 
after drug-eluting stent implantation (ARCTIC-Interruption): a randomised trial. Lancet 2014;384:1577-1585.

113. Bittl JA, Baber U, Bradley SM, Wijeysundera DN. Duration of dual antiplatelet therapy: a systematic review for the 2016 ACC/AHA Guideline Focused Update on Duration of Dual Antiplatelet Therapy in Patients With Coronary Artery Disease: A Report of the American College of Cardiology/American Heart Association Task Force on Clinical Practice Guidelines. J Am Coll Cardiol 2016;68:1116-1139.

114. Valgimigli M, Borghesi M, Tebaldi M, Vranckx P, Parrinello G, Ferrari R. Should duration of dual antiplatelet therapy depend on the type and/or potency of implanted stent? A pre-specified analysis from the PROlonging Dual antiplatelet treatment after Grading stent-induced Intimal hyperplasia studY (PRODIGY). Eur Heart J 2013;34:909-919.

115. Hermiller JB, Krucoff MW, Kereiakes DJ, Windecker S, Steg PG, Yeh RW, Cohen DJ, Cutlip DE, Massaro JM, Hsieh WH, Mauri L, DAPT Study Investigators. Benefits and risks of extended dual antiplatelet therapy after everolimus-eluting stents. JACC Cardiovasc Interv 2016;9:138-147.

116. Sharma A, Sharma SK, Vallakati A, Garg A, Lavie CJ, Mukherjee D, Marmur JD. Duration of dual antiplatelet therapy after various drug-eluting stent implantation. Int J Cardiol 2016;215:157-166.

117. Palmerini T, Stone GW. Optimal duration of dual antiplatelet therapy after drug-eluting stent implantation: conceptual evolution based on emerging evidence. Eur Heart J 2016;37:353-364.

118. Ellis SG, Kereiakes DJ, Metzger DC, Caputo RP, Rizik DG, Teirstein PS, Litt MR, Kini A, Kabour A, Marx SO, Popma JJ, McGreevy R, Zhang Z, Simonton C, Stone GW, ABSORB III Investigators. Everolimus-eluting bioresorbable scaffolds for coronary artery disease. N Engl J Med 2015;373:1905-1915.

119. Cassese S, Byrne RA, Ndrepepa G, Kufner S, Wiebe J, Repp J, Schunkert H, Fusaro M, Kimura T, Kastrati A. Everolimus-eluting bioresorbable vascular scaffolds versus everolimus-eluting metallic stents: a meta-analysis of randomised controlled trials. Lancet 2016;387:537-544.

120. Serruys PW, Chevalier B, Sotomi Y, Cequier A, Carrie D, Piek JJ, Van Boven AJ, Dominici M, Dudek D, McClean D, Helqvist S, Haude M, Reith S, de Sousa Almeida M, Campo G, Iniguez A, Sabate M, Windecker S, Onuma Y. Comparison of an everolimus-eluting bioresorbable scaffold with an everolimus-eluting metallic stent for the treatment of coronary artery stenosis (ABSORB II): a 3 year, randomised, controlled, single-blind, multicentre clinical trial. Lancet 2016;388:2479-2491.

121. Räber L, Brugaletta S, Yamaji K, O’Sullivan CJ, Otsuki S, Koppara T, Taniwaki M, Onuma Y, Freixa X, Eberli FR, Serruys PW, Joner M, Sabate M, Windecker S. Very late scaffold thrombosis: intracoronary imaging and histopathological and spectroscopic findings. J Am Coll Cardiol 2015;66:1901-1914.

122. Alfonso F, Perez-Vizcayno MJ, Cardenas A, Garcia del Blanco B, GarciaTouchard A, Lopez-Minguez JR, Benedicto A, Masotti M, Zueco J, Iniguez A, Velazquez M, Moreno R, Mainar V, Dominguez A, Pomar F, Melgares R, Rivero F, Jimenez-Quevedo P, Gonzalo N, Fernandez C, Macaya C. A prospective randomized trial of drug-eluting balloons versus everolimus-eluting stents in patients with in-stent restenosis of drug-eluting stents: the RIBS IV randomized clinical trial. J Am Coll Cardiol 2015;66:23-33.

123. Xu B, Gao R, Wang J, Yang Y, Chen S, Liu B, Chen F, Li Z, Han Y, Fu G, Zhao $Y, \mathrm{Ge}$. A prospective, multicenter, randomized trial of paclitaxel-coated balloon versus paclitaxel-eluting stent for the treatment of drug-eluting stent instent restenosis: results from the PEPCAD China ISR trial. JACC Cardiovasc Interv 2014;7:204-211.

124. Byrne RA, Neumann FJ, Mehilli J, Pinieck S, Wolff B, Tiroch K, Schulz S, Fusaro M, Ott I, Ibrahim T, Hausleiter J, Valina C, Pache J, Laugwitz KL, Massberg S, Kastrati A. Paclitaxel-eluting balloons, paclitaxel-eluting stents, and balloon angioplasty in patients with restenosis after implantation of a drug-eluting stent (ISAR-DESIRE 3): a randomised, open-label trial. Lancet 2013;381:461-467.

125. Wöhrle J, Zadura M, Mobius-Winkler S, Leschke M, Opitz C, Ahmed W, Barragan P, Simon JP, Cassel G, Scheller B. SeQuent Please World Wide Registry: clinical results of SeQuent Please paclitaxel-coated balloon angioplasty in a large-scale, prospective registry study. I Am Coll Cardiol 2012;18:1733-1738.

126. Han $Y$, Xu B, Xu K, Guan C, Jing Q, Zheng Q, Li X, Zhao X, Wang H, Zhao X, Li X, Yu P, Zang H, Wang Z, Cao X, Zhang J, Pang W, Li J, Yang Y, Dangas GD. Six versus 12 months of dual antiplatelet therapy after implantation of biodegradable polymer sirolimus-eluting stent: randomized substudy of the I-LOVEIT 2 trial. Circ Cardiovasc Interv 2016;9:e003145.

127. Hong SJ, Shin DH, Kim JS, Kim BK, Ko YG, Choi D, Her AY, Kim YH, Jang Y, Hong MK. 6-month versus 12-month dual-antiplatelet therapy following long everolimus-eluting stent implantation: the IVUS-XPL randomized clinical trial. JACC Cardiovasc Interv 2016;9:1438-1446.

128. Kereiakes DJ, Yeh RW, Massaro JM, Driscoll-Shempp P, Cutlip DE, Steg PG, Gershlick AH, Darius H, Meredith IT, Ormiston J, Tanguay JF, Windecker S, Garratt KN, Kandzari DE, Lee DP, Simon DI, lancu AC, Trebacz J, Mauri L,
Dual Antiplatelet Therapy (DAPT) Study Investigators. Antiplatelet therapy duration following bare metal or drug-eluting coronary stents: the dual antiplatelet therapy randomized clinical trial. JAMA 2015;313:1113-1121.

129. Urban P, Meredith IT, Abizaid A, Pocock SJ, Carrie D, Naber C, Lipiecki J, Richardt G, Iniguez A, Brunel P, Valdes-Chavarri M, Garot P, Talwar S, Berland J, Abdellaoui M, Eberli F, Oldroyd K, Zambahari R, Gregson J, Greene S, Stoll HP, Morice MC, LEADERS FREE Investigators. Polymer-free drug-coated coronary stents in patients at high bleeding risk. N Engl J Med 2015;373:2038-2047.

130. Valgimigli M, Patialiakas A, Thury A, McFadden E, Colangelo S, Campo G, Tebaldi M, Ungi I, Tondi S, Roffi M, Menozzi A, de Cesare N, Garbo R, Meliga E, Testa L, Gabriel HM, Airoldi F, Ferlini M, Liistro F, Dellavalle A, Vranckx P, Briguori C, ZEUS Investigators. Zotarolimus-eluting versus bare-metal stents in uncertain drug-eluting stent candidates. J Am Coll Cardiol 2015;65:805-815.

131. Valgimigli M, Sabate M, Kaiser C, Brugaletta S, de la Torre Hernandez JM, Galatius S, Cequier A, Eberli F, de Belder A, Serruys PW, Ferrante G. Effects of cobaltchromium everolimus eluting stents or bare metal stent on fatal and non-fatal cardiovascular events: patient level meta-analysis. BMJ 2014;349:g6427.

132. Bonaa KH, Mannsverk J, Wiseth R, Aaberge L, Myreng Y, Nygard O, Nilsen DW, Klow NE, Uchto M, Trovik T, Bendz B, Stavnes S, Bjornerheim R, Larsen Al, Slette M, Steigen T, Jakobsen OJ, Bleie O, Fossum E, Hanssen TA, DahlEriksen O, Njolstad I, Rasmussen K, Wilsgaard T, Nordrehaug JE, Norstent Investigators. Drug-eluting or bare-metal stents for coronary artery disease. N Engl J Med 2016;375:1242-1252.

133. Alfonso F, Perez-Vizcayno MJ, Cardenas A, Garcia Del Blanco B, Seidelberger B, Iniguez A, Gomez-Recio M, Masotti M, Velazquez MT, Sanchis J, GarciaTouchard A, Zueco J, Bethencourt A, Melgares R, Cequier A, Dominguez A, Mainar V, Lopez-Minguez JR, Moreu J, Marti V, Moreno R, Jimenez-Quevedo P, Gonzalo N, Fernandez C, Macaya C. A randomized comparison of drug-eluting balloon versus everolimus-eluting stent in patients with bare-metal stent-instent restenosis: the RIBS $\vee$ Clinical Trial (Restenosis Intra-stent of Bare Metal Stents: paclitaxel-eluting balloon vs. everolimus-eluting stent). J Am Coll Cardiol 2014;63:1378-1386.

134. Byrne RA, Serruys PW, Baumbach A, Escaned J, Fajadet J, James S, Joner M, Oktay S, Juni P, Kastrati A, Sianos G, Stefanini GG, Wijns W, Windecker S. Report of a European Society of Cardiology-European Association of Percutaneous Cardiovascular Interventions task force on the evaluation of coronary stents in Europe: executive summary. Eur Heart J 2015;36:2608-2620.

135. Bhatt DL, Flather MD, Hacke W, Berger PB, Black HR, Boden WE, Cacoub P, Cohen EA, Creager MA, Easton JD, Hamm CW, Hankey GJ, Johnston SC, Mak $\mathrm{KH}$, Mas JL, Montalescot G, Pearson TA, Steg PG, Steinhubl SR, Weber MA, Fabry-Ribaudo L, Hu T, Topol EJ, Fox KA, CHARISMA Investigators. Patients with prior myocardial infarction, stroke, or symptomatic peripheral arterial disease in the CHARISMA trial. J Am Coll Cardiol 2007;49:1982-1988.

136. Wiviott SD, White HD, Ohman EM, Fox KA, Armstrong PW, Prabhakaran D, Hafley G, Lokhnygina Y, Boden WE, Hamm C, Clemmensen P, Nicolau JC, Menozzi A, Ruzyllo W, Widimsky P, Oto A, Leiva-Pons J, Pavlides G, Winters KJ, Roe MT, Bhatt DL. Prasugrel versus clopidogrel for patients with unstable angina or non-ST-segment elevation myocardial infarction with or without angiography: a secondary, prespecified analysis of the TRILOGY ACS trial. Lancet 2013;382:605-613.

137. Scirica BM, Bonaca MP, Braunwald E, De Ferrari GM, Isaza D, Lewis BS, Mehrhof F, Merlini PA, Murphy SA, Sabatine MS, Tendera M, Van de Werf F, Wilcox R, Morrow DA, TRA $2^{\circ}$ P-TIMI 50 Steering Committee Investigators. Vorapaxar for secondary prevention of thrombotic events for patients with previous myocardial infarction: a prespecified subgroup analysis of the TRA $2^{\circ} \mathrm{P}$-TIMI 50 trial. Lancet 2012;380:1317-1324.

138. Ndrepepa G, Berger PB, Mehilli J, Seyfarth M, Neumann FJ, Schomig A, Kastrati A. Periprocedural bleeding and 1-year outcome after percutaneous coronary interventions: appropriateness of including bleeding as a component of a quadruple end point. J Am Coll Cardiol 2008;51:690-697.

139. Bonaca MP, Bhatt DL, Steg PG, Storey RF, Cohen M, Im K, Oude Ophuis T, Budaj A, Goto S, Lopez-Sendon J, Diaz R, Dalby A, Van de Werf F, Ardissino D, Montalescot G, Aylward P, Magnani G, Jensen EC, Held P, Braunwald E, Sabatine MS. Ischaemic risk and efficacy of ticagrelor in relation to time from P2Y12 inhibitor withdrawal in patients with prior myocardial infarction: insights from PEGASUS-TIMI 54. Eur Heart J 2016;37:1133-1142.

140. Bonaca MP, Bhatt DL, Storey RF, Steg PG, Cohen M, Kuder J, Goodrich E, Nicolau JC, Parkhomenko A, Lopez-Sendon J, Dellborg M, Dalby A, Spinar J, Aylward P, Corbalan R, Abola MT, Jensen EC, Held P, Braunwald E, Sabatine MS. Ticagrelor for prevention of ischemic events after myocardial infarction in patients with peripheral artery disease. J Am Coll Cardiol 2016;67:2719-2728.

141. Udell JA, Bonaca MP, Collet JP, Lincoff AM, Kereiakes DJ, Costa F, Lee CW, Mauri L, Valgimigli M, Park SJ, Montalescot G, Sabatine MS, Braunwald E, Bhatt DL. Long-term dual antiplatelet therapy for secondary prevention of cardiovascular events in the subgroup of patients with previous myocardial infarction: a collaborative meta-analysis of randomized trials. Eur Heart J 2016;37:390-399. 
142. Costa F, Adamo M, Ariotti S, Navarese EP, Biondi-Zoccai G, Valgimigli M. Impact of greater than 12-month dual antiplatelet therapy duration on mortality: drug-specific or a class-effect? A meta-analysis. Int J Cardiol 2015;201:179-181.

143. Palmerini T, Della Riva D, Benedetto U, Reggiani LB, Feres F, Abizaid A, Gilard M, Morice M, Valgimigli M, Hong M, Kim B, Jang Y, Kim H, Park KW, Colombo A, Chieffo A, Sangiorgi D, Biondi-Zoccai G, Genereux P, Angelini GD, White, Bhatt DL, Stone GW. Three, six or twelve months of dual antiplatelet therapy after drug-eluting stent implantation in patients with or without acute coronary syndromes: an individual patient data pairwise and network meta-analysis of six randomized trials and 11473 patients. Eur Heart / 2017;38:1034-1043.

144. Magnani G, Storey RF, Steg G, Bhatt DL, Cohen M, Kuder J, Im K, Aylward P, Ardissino D, Isaza D, Parkhomenko A, Goudev AR, Dellborg M, Kontny F, Corbalan R, Medina F, Jensen EC, Held P, Braunwald E, Sabatine MS, Bonaca MP. Efficacy and safety of ticagrelor for long-term secondary prevention of atherothrombotic events in relation to renal function: insights from the PEGASUS-TIMI 54 trial. Eur Heart J 2016;37:400-408.

145. Bhatt DL, Bonaca MP, Bansilal S, Angiolillo DJ, Cohen M, Storey RF, Im K, Murphy SA, Held P, Braunwald E, Sabatine MS, Steg PG. Reduction in ischemic events with ticagrelor in diabetic patients with prior myocardial infarction in PEGASUS-TIMI 54. J Am Coll Cardiol 2016;67:2732-2740.

146. Vranckx P, Valgimigli M, Windecker S, Steg PG, Hamm C, Juni P, Garcia-Garcia $H M$, van Es GA, Serruys PW. Long-term ticagrelor monotherapy versus standard dual antiplatelet therapy followed by aspirin monotherapy in patients undergoing biolimus-eluting stent implantation: rationale and design of the GLOBAL LEADERS trial. Eurolntervention 2015;12:1239-1245.

147. Fox KA, Mehta SR, Peters R, Zhao F, Lakkis N, Gersh BJ, Yusuf S. Benefits and risks of the combination of clopidogrel and aspirin in patients undergoing surgical revascularization for non-ST-elevation acute coronary syndrome: the Clopidogrel in Unstable angina to prevent Recurrent ischemic Events (CURE) Trial. Circulation 2004;110:1202-1208.

148. Verma S, Goodman SG, Mehta SR, Latter DA, Ruel M, Gupta M, Yanagawa B, Al-Omran M, Gupta N, Teoh H, Friedrich JO. Should dual antiplatelet therapy be used in patients following coronary artery bypass surgery? A meta-analysis of randomized controlled trials. BMC Surg 2015;15:112

149. Deo SV, Dunlay SM, Shah IK, Altarabsheh SE, Erwin PJ, Boilson BA, Park SJ, Joyce LD. Dual anti-platelet therapy after coronary artery bypass grafting: is there any benefit? A systematic review and meta-analysis. I Card Surg 2013;28:109-116

150. Held C, Asenblad N, Bassand JP, Becker RC, Cannon CP, Claeys MJ, Harrington RA, Horrow J, Husted S, James SK, Mahaffey KW, Nicolau JC, Scirica BM, Storey RF, Vintila M, Ycas J, Wallentin L. Ticagrelor versus clopidogrel in patients with acute coronary syndromes undergoing coronary artery bypass surgery: results from the PLATO (Platelet Inhibition and Patient Outcomes) trial. J Am Coll Cardiol 2011;57:672-684.

151. Smith PK, Goodnough LT, Levy JH, Poston RS, Short MA, Weerakkody G], Lenarz LA. Mortality benefit with prasugrel in the TRITON-TIMI 38 coronary artery bypass grafting cohort: risk-adjusted retrospective data analysis. J Am Coll Cardiol 2012;60:388-396.

152. Hansson EC, Jideus L, Aberg B, Bjursten H, Dreifaldt M, Holmgren A, Ivert T, Nozohoor S, Barbu M, Svedjeholm R, Jeppsson A. Coronary artery bypass grafting-related bleeding complications in patients treated with ticagrelor or clopidogrel: a nationwide study. Eur Heart J 2016;37:189-197.

153. Tomsic A, Schotborgh MA, Manshanden JS, Li WW, de Mol BA. Coronary artery bypass grafting-related bleeding complications in patients treated with dual antiplatelet treatment. Eur J Cardiothorac Surg 2016;50:849-856.

154. Pickard AS, Becker RC, Schumock GT, Frye CB. Clopidogrel-associated bleeding and related complications in patients undergoing coronary artery bypass grafting. Pharmacotherapy 2008;28:376-392.

155. Purkayastha S, Athanasiou T, Malinovski V, Tekkis P, Foale R, Casula R, Glenville B, Darzi A. Does clopidogrel affect outcome after coronary artery bypass grafting? A meta-analysis. Heart 2006;92:531-532.

156. Windecker S, Kolh P, Alfonso F, Collet JP, Cremer J, Falk V, Filippatos G, Hamm C, Head SJ, Juni P, Kappetein AP, Kastrati A, Knuuti J, Landmesser U, Laufer G, Neumann FJ, Richter DJ, Schauerte P, Sousa Uva M, Stefanini GG, Taggart DP, Torracca L, Valgimigli M, Wijns W, Witkowski A. 2014 ESC/ EACTS Guidelines on myocardial revascularization: The Task Force on Myocardial Revascularization of the European Society of Cardiology (ESC) and the European Association for Cardio-Thoracic Surgery (EACTS) Developed with the special contribution of the European Association of Percutaneous Cardiovascular Interventions (EAPCI). Eur Heart J 2014;35:2541-2619.

157. Ferraris VA, Saha SP, Oestreich JH, Song HK, Rosengart T, Reece TB, Mazer CD, Bridges CR, Despotis GJ, Jointer K, Clough ER, Society of Thoracic Surgeons. 2012 update to the Society of Thoracic Surgeons guideline on use of antiplatelet drugs in patients having cardiac and noncardiac operations. Ann Thorac Surg 2012;94:1761-1781.
158. Wallentin L. P2Y(12) inhibitors: differences in properties and mechanisms of action and potential consequences for clinical use. Eur Heart J 2009;30:1964-1977.

159. Gurbel PA, Bliden KP, Butler K, Tantry US, Gesheff T, Wei C, Teng R, Antonino MJ, Patil SB, Karunakaran A, Kereiakes DJ, Parris C, Purdy D, Wilson $\checkmark$, Ledley GS, Storey RF. Randomized double-blind assessment of the ONSET and OFFSET of the antiplatelet effects of ticagrelor versus clopidogrel in patients with stable coronary artery disease: the ONSET/OFFSET study. Circulation 2009;120:2577-2585.

160. Gherli R, Mariscalco G, Dalen M, Onorati F, Perrotti A, Chocron S, Verhoye JP, Gulbins H, Reichart D, Svenarud P, Faggian G, Santarpino G, Fischlein T, Maselli D, Dominici C, Musumeci F, Rubino AS, Mignosa C, De Feo M, Bancone C, Gatti G, Maschietto L, Santini F, Nicolini F, Gherli T, Zanobini M, Kinnunen EM, Ruggieri VG, Rosato S, Biancari F. Safety of preoperative use of ticagrelor with or without aspirin compared with aspirin alone in patients with acute coronary syndromes undergoing coronary artery bypass grafting. JAMA Cardiol 2016;1:921-928.

161. Steg PG, James SK, Atar D, Badano LP, Blomstrom-Lundqvist C, Borger MA, D Mario C, Dickstein K, Ducrocq G, Fernandez-Aviles F, Gershlick AH, Giannuzzi P, Halvorsen S, Huber K, Juni P, Kastrati A, Knuuti J, Lenzen MJ, Mahaffey KW, Valgimigli M, van 't Hof A, Widimsky P, Zahger D. ESC Guidelines for the management of acute myocardial infarction in patients presenting with ST-segment elevation. Eur Heart J 2012;33:2569-2619.

162. Kirchhof P, Benussi S, Kotecha D, Ahlsson A, Atar D, Casadei B, Castella M, Diener HC, Heidbuchel H, Hendriks J, Hindricks G, Manolis AS, Oldgren J, Popescu BA, Schotten U, Van Putte B, Vardas P. 2016 ESC Guidelines for the management of atrial fibrillation developed in collaboration with EACTS: The Task Force for the management of atrial fibrillation of the European Society of Cardiology (ESC) developed with the special contribution of the European Heart Rhythm Association (EHRA) of the ESC Endorsed by the European Stroke Organisation (ESO). Eur Heart J 2016;37:2893-2962.

163. Hastings S, Myles P, Mcllroy D. Aspirin and coronary artery surgery: a systematic review and meta-analysis. Br J Anaesth 2015;115:376-385.

164. Myles PS, Smith JA, Forbes A, Silbert B, Jayarajah M, Painter T, Cooper DJ, Marasco S, McNeil J, Bussieres JS, Wallace S, Atacas Investigators of the ANZCA Clinical Trials Network. Stopping vs. continuing aspirin before coronary artery surgery. N EnglJ Med 2016;374:728-737.

165. Dacey LJ, Munoz JJ, Johnson ER, Leavitt BJ, Maloney CT, Morton JR, Olmstead EM, Birkmeyer JD, O'Connor GT, the Northern New England Cardiovascular Disease Study Group. Effect of preoperative aspirin use on mortality in coronary artery bypass grafting patients. Ann Thorac Surg 2000;70:1986-1990.

166. Hansson EC, Shams Hakimi C, Astrom-Olsson K, Hesse C, Wallen H, Dellborg M, Albertsson P, Jeppsson A. Effects of ex vivo platelet supplementation on platelet aggregability in blood samples from patients treated with acetylsalicylic acid, clopidogrel, or ticagrelor. BrJ Anaesth 2014;112:570-575.

167. Martin AC, Berndt C, Calmette L, Philip I, Decouture B, Gaussem P, GouinThibault I, Samama CM, Bachelot-Loza C, Godier A. The effectiveness of platelet supplementation for the reversal of ticagrelor-induced inhibition of platelet aggregation: an in-vitro study. Eur J Anaesthesiol 2016;33:361-367.

168. O'Connor SA, Amour J, Mercadier A, Martin R, Kerneis M, Abtan J, Brugier D Silvain J, Barthelemy O, Leprince P, Montalescot G, Collet JP; ACTION Study Group. Efficacy of ex vivo autologous and in vivo platelet transfusion in the reversal of P2Y12 inhibition by clopidogrel, prasugrel, and ticagrelor: the APTITUDE study. Circ Cardiovasc Interv 2015;8:e002786.

169. Malm C, Hansson E, Åkesson J, Andersson M, Hesse C, Shams Hakimi C Jeppsson A. Preoperative platelet function predicts perioperative bleeding complications in ticagrelor-treated cardiac surgery patients: a prospective observational study. Br J Anaesth 2016;117:309-315.

170. Storey RF, Bliden KP, Ecob R, Karunakaran A, Butler K, Wei C, Tantry U Gurbel PA. Earlier recovery of platelet function after discontinuation of treatment with ticagrelor compared with clopidogrel in patients with high antiplatelet responses. J Thromb Haemost 2011;9:1730-1737.

171. Hansson EC, Malm CJ, Hesse C, Hornestam B, Dellborg M, Rexius H, Jeppsson A. Platelet function recovery after ticagrelor withdrawal in patients awaiting urgent coronary surgery. Eur J Cardiothorac Surg 2016;51:633-637.

172. Kwak YL, Kim JC, Choi YS, Yoo KJ, Song Y, Shim JK. Clopidogrel responsiveness regardless of the discontinuation date predicts increased blood loss and transfusion requirement after off-pump coronary artery bypass graft surgery. J Am Coll Cardiol 2010;56:1994-2002.

173. Ranucci M, Baryshnikova E, Soro G, Ballotta A, De Benedetti D, Conti D. Multiple electrode whole-blood aggregometry and bleeding in cardiac surgery patients receiving thienopyridines. Ann Thorac Surg 2011;91:123-129.

174. Ranucci M, Colella D, Baryshnikova E, Di Dedda U. Effect of preoperative P2Y12 and thrombin platelet receptor inhibition on bleeding after cardiac surgery. BrJ Anaesth 2014;113:970-976.

175. Mahla E, Suarez TA, Bliden KP, Rehak P, Metzler H, Sequeira AJ, Cho P, Sell J, Fan J, Antonino MJ, Tantry US, Gurbel PA. Platelet function measurementbased strategy to reduce bleeding and waiting time in clopidogrel-treated 
patients undergoing coronary artery bypass graft surgery: the timing based on platelet function strategy to reduce clopidogrel-associated bleeding related to CABG (TARGET-CABG) study. Circ Cardiovasc Interv 2012;5:261-269.

176. Vries MJ, Bouman HJ, Olie RH, Veenstra LF, Zwaveling S, Verhezen PW, Ten Cate-Hoek AJ, Ten Cate H, Henskens YM, van der Meijden PE. Determinants of agreement between proposed therapeutic windows of platelet function tests in vulnerable patients. Eur Heart J Cardiovasc Pharmacother 2017;3:11-17.

177. Nocerino AG, Achenbach S, Taylor AJ. Meta-analysis of effect of single versus dual antiplatelet therapy on early patency of bypass conduits after coronary artery bypass grafting. Am J Cardiol 2013;112:1576-1579.

178. Mannacio VA, Di Tommaso L, Antignan A, De Amicis V, Vosa C. Aspirin plus clopidogrel for optimal platelet inhibition following off-pump coronary artery bypass surgery: results from the CRYSSA (prevention of Coronary arteRY bypaSS occlusion After off-pump procedures) randomised study. Heart 2012;98:1710-1715.

179. Morrow DA, Braunwald E, Bonaca MP, Ameriso SF, Dalby AJ, Fish MP, Fox KA, Lipka LJ, Liu X, Nicolau JC, Ophuis AJ, Paolasso E, Scirica BM, Spinar J, Theroux $\mathrm{P}$, Wiviott SD, Strony J, Murphy SA. Vorapaxar in the secondary prevention of atherothrombotic events. N Engl J Med 2012;366:1404-1413.

180. James SK, Roe MT, Cannon CP, Cornel JH, Horrow J, Husted S, Katus H, Morais J, Steg PG, Storey RF, Stevens S, Wallentin L, Harrington RA, PLATO Investigators. Ticagrelor versus clopidogrel in patients with acute coronary syndromes intended for non-invasive management: substudy from prospective randomised PLATelet inhibition and patient Outcomes (PLATO) trial. BMJ 2011;342:d3527.

181. Prami T, Khanfir H, Deleskog A, Hasvold P, Kyto V, Reissell E, Airaksinen J. Clinical factors associated with initiation of and persistence with ADP receptorinhibiting oral antiplatelet treatment after acute coronary syndrome: a nationwide cohort study from Finland. BMJ Open 2016;6:e012604.

182. lannaccone M, Quadri G, Taha S, D'Ascenzo F, Montefusco A, Omede P, Jang IK, Niccoli G, Souteyrand G, Yundai C, Toutouzas K, Benedetto S, Barbero U, Annone U, Lonni E, Imori Y, Biondi-Zoccai G, Templin C, Moretti C, Luscher TF, Gaita F. Prevalence and predictors of culprit plaque rupture at OCT in patients with coronary artery disease: a meta-analysis. Eur Heart J Cardiovasc Imaging 2016;17:1128-1137.

183. Sorensen R, Hansen ML, Abildstrom SZ, Hvelplund A, Andersson C, Jorgensen C, Madsen JK, Hansen PR, Kober L, Torp-Pedersen C, Gislason $\mathrm{GH}$. Risk of bleeding in patients with acute myocardial infarction treated with different combinations of aspirin, clopidogrel, and vitamin $\mathrm{K}$ antagonists in Denmark: a retrospective analysis of nationwide registry data. Lancet 2009;374:1967-1974

184. Hansen ML, Sorensen R, Clausen MT, Fog-Petersen ML, Raunso J, Gadsboll N, Gislason GH, Folke F, Andersen SS, Schramm TK, Abildstrom SZ, Poulsen HE, Kober L, Torp-Pedersen C. Risk of bleeding with single, dual, or triple therapy with warfarin, aspirin, and clopidogrel in patients with atrial fibrillation. Arch Intern Med 2010;170:1433-1441.

185. Dans AL, Connolly SJ, Wallentin L, Yang S, Nakamya J, Brueckmann M, Ezekowitz M, Oldgren J, Eikelboom JW, Reilly PA, Yusuf S. Concomitant use of antiplatelet therapy with dabigatran or warfarin in the Randomized Evaluation of Long-Term Anticoagulation Therapy (RE-LY) trial. Circulation 2013;127:634-640.

186. Oldgren J, Budaj A, Granger CB, Khder Y, Roberts J, Siegbahn A, Tijssen JG, Van de Werf F, Wallentin L. Dabigatran vs. placebo in patients with acute coronary syndromes on dual antiplatelet therapy: a randomized, double-blind, phase II trial. Eur Heart J 2011;32:2781-2789.

187. Lopes RD, Al-Khatib SM, Wallentin L, Yang H, Ansell J, Bahit MC, De Caterina R, Dorian P, Easton JD, Erol C, Ezekowitz JA, Gersh BJ, Granger CB, Hohnloser $\mathrm{SH}$, Horowitz J, Hylek EM, McMurray JJ, Mohan P, Vinereanu D, Alexander JH. Efficacy and safety of apixaban compared with warfarin according to patient risk of stroke and of bleeding in atrial fibrillation: a secondary analysis of a randomised controlled trial. Lancet 2012;380:1749-1758.

188. Barnes GD, Gu X, Haymart B, Kline-Rogers E, Almany S, Kozlowski J, Besley D, Krol GD, Froehlich JB, Kaatz S. The predictive ability of the CHADS2 and CHA2DS2-VASc scores for bleeding risk in atrial fibrillation: the MAQI(2) experience. Thromb Res 2014;134:294-299.

189. Roldan V, Marin F, Manzano-Fernandez S, Gallego P, Vilchez JA, Valdes M, Vicente V, Lip GY. The HAS-BLED score has better prediction accuracy for major bleeding than CHADS2 or CHA2DS2-VASc scores in anticoagulated patients with atrial fibrillation. I Am Coll Cardiol 2013;62:2199-2204.

190. Hijazi Z, Oldgren J, Lindback J, Alexander JH, Connolly SJ, Eikelboom JW, Ezekowitz MD, Held C, Hylek EM, Lopes RD, Siegbahn A, Yusuf S, Granger CB, Wallentin L. The novel biomarker-based ABC (age, biomarkers, clinical history)-bleeding risk score for patients with atrial fibrillation: a derivation and validation study. Lancet 2016;387:2302-2311.

191. Gibson CM, Mehran R, Bode C, Halperin J, Verheugt FW, Wildgoose P, Birmingham M, lanus J, Burton P, van Eickels M, Korjian S, Daaboul Y, Lip GYH,
Cohen M, Husted S, Peterson ED, Fox KA. Prevention of bleeding in patients with atrial fibrillation undergoing PCI. N Engl J Med 2016;375:2423-2434

192. Sarafoff N, Martischnig A, Wealer J, Mayer K, Mehilli J, Sibbing D, Kastrati A. Triple therapy with aspirin, prasugrel, and vitamin $\mathrm{K}$ antagonists in patients with drug-eluting stent implantation and an indication for oral anticoagulation. J Am Coll Cardiol 2013:61:2060-2066.

193. Dewilde WJ, Oirbans T, Verheugt FW, Kelder JC, De Smet BJ, Herrman JP, Adriaenssens T, Vrolix M, Heestermans AA, Vis MM, Tijsen JG, van 't Hof AW, ten Berg JM, WOEST Study Investigators. Use of clopidogrel with or without aspirin in patients taking oral anticoagulant therapy and undergoing percutaneous coronary intervention: an open-label, randomised, controlled trial. Lancet 2013;381:1107-1115.

194. Gibson CM, Pinto DS, Chi G, Arbetter D, Yee M, Mehran R, Bode C, Halperin J, Verheugt FWA, Wildgoose P, Burton P, van Eickels M, Korjian S, Daaboul Y, Jain P, Lip GYH, Cohen M, Peterson ED, Fox KAA. Recurrent hospitalization among patients with atrial fibrillation undergoing intracoronary stenting treated with 2 treatment strategies of rivaroxaban or a dose-adjusted oral vitamin $\mathrm{K}$ antagonist treatment strategy. Circulation 2017;135:323-333.

195. Fiedler KA, Maeng M, Mehilli J, Schulz-Schupke S, Byrne RA, Sibbing D, Hoppmann P, Schneider S, Fusaro M, Ott I, Kristensen SD, Ibrahim T, Massberg S, Schunkert H, Laugwitz KL, Kastrati A, Sarafoff N. Duration of triple therapy in patients requiring oral anticoagulation after drug-eluting stent implantation: the ISAR-TRIPLE trial. J Am Coll Cardiol 2015;65:1619-1629.

196. Lamberts M, Olesen JB, Ruwald MH, Hansen CM, Karasoy D, Kristensen SL, Kober L, Torp-Pedersen C, Gislason GH, Hansen ML. Bleeding after initiation of multiple antithrombotic drugs, including triple therapy, in atrial fibrillation patients following myocardial infarction and coronary intervention: a nationwide cohort study. Circulation 2012;126:1185-1193.

197. Lamberts M, Gislason GH, Olesen JB, Kristensen SL, Schjerning Olsen AM, Mikkelsen A, Christensen CB, Lip GY, Kober L, Torp-Pedersen C, Hansen ML. Oral anticoagulation and antiplatelets in atrial fibrillation patients after myocardial infarction and coronary intervention. J Am Coll Cardiol 2013;62:981-989.

198. Lamberts M, Gislason GH, Lip GY, Lassen JF, Olesen JB, Mikkelsen AP, Sorensen R, Kober L, Torp-Pedersen C, Hansen ML. Antiplatelet therapy for stable coronary artery disease in atrial fibrillation patients taking an oral anticoagulant: a nationwide cohort study. Circulation 2014;129:1577-1585.

199. Connolly SJ, Ezekowitz MD, Yusuf S, Eikelboom J, Oldgren J, Parekh A, Pogue J, Reilly PA, Themeles E, Varrone J, Wang S, Alings M, Xavier D, Zhu J, Diaz R, Lewis BS, Darius H, Diener HC, Joyner CD, Wallentin L; RE-LY Steering Committee and Investigators. Dabigatran versus warfarin in patients with atrial fibrillation. N Engl J Med 2009;361:1139-1151.

200. Patel MR, Mahaffey KW, Garg J, Pan G, Singer DE, Hacke W, Breithardt G, Halperin JL, Hankey GJ, Piccini JP, Becker RC, Nessel CC, Paolini JF, Berkowitz SD, Fox KA, Califf RM, for the ROCKET-AF Study Investigators. Rivaroxaban versus warfarin in nonvalvular atrial fibrillation. $N$ Engl J Med 2011;365:883-891.

201. Granger CB, Alexander JH, McMurray JJ, Lopes RD, Hylek EM, Hanna M, AlKhalidi HR, Ansell J, Atar D, Avezum A, Bahit MC, Diaz R, Easton JD, Ezekowitz JA, Flaker G, Garcia D, Geraldes M, Gersh BJ, Golitsyn S, Goto S, Hermosillo AG, Hohnloser SH, Horowitz J, Mohan P, Jansky P, Lewis BS, Lopez-Sendon JL, Pais P, Parkhomenko A, Verheugt FW, Zhu J, Wallentin L; ARISTOTLE Committees and Investigators. Apixaban versus warfarin in patients with atria fibrillation. N Engl J Med 2011;365:981-992.

202. Giugliano RP, Ruff CT, Braunwald E, Murphy SA, Wiviott SD, Halperin JL, Waldo AL, Ezekowitz MD, Weitz JI, Spinar J, Ruzyllo W, Ruda M, Koretsune Y, Betcher J, Shi M, Grip LT, Patel SP, Patel I, Hanyok J], Mercuri M, Antman EM, for the ENGAGE-AF-TIMI 48 Study Investigators. Edoxaban versus warfarin in patients with atrial fibrillation. N Engl J Med 2013;369:2093-2104.

203. Hawn MT, Graham LA, Richman JS, Itani KM, Henderson WG, Maddox TM. Risk of major adverse cardiac events following noncardiac surgery in patients with coronary stents. JAMA 2013;310:1462-1472.

204. Ariotti S, Adamo M, Costa F, Patialiakas A, Briguori C, Thury A, Colangelo S, Campo G, Tebaldi M, Ungi I, Tondi S, Roffi M, Menozzi A, de Cesare N, Garbo R, Meliga E, Testa L, Gabriel HM, Ferlini M, Vranckx P, Valgimigli M, ZEUS Investigators. Is bare-metal stent implantation still justifiable in high bleeding risk patients undergoing percutaneous coronary intervention?: A pre-specified analysis from the ZEUS trial. JACC Cardiovasc Interv 2016;9:426-436.

205. Kristensen SD, Knuuti J, Saraste A, Anker S, Botker HE, Hert SD, Ford I, Gonzalez-Juanatey JR, Gorenek B, Heyndrickx GR, Hoeft A, Huber K, lung B, Kjeldsen KP, Longrois D, Luscher TF, Pierard L, Pocock S, Price S, Roffi M, Sirnes PA, Sousa-Uva M, Voudris V, Funck-Brentano C; Authors/Task Force Members. 2014 ESC/ESA Guidelines on non-cardiac surgery: cardiovascular assessment and management: The Joint Task Force on non-cardiac surgery: cardiovascular assessment and management of the European Society of Cardiology (ESC) and the European Society of Anaesthesiology (ESA). Eur Heart J 2014;35:2383-2431. 
206. Chee YL, Crawford JC, Watson HG, Greaves M. Guidelines on the assessment of bleeding risk prior to surgery or invasive procedures. British Committee for Standards in Haematology. Br J Haematol 2008;140:496-504.

207. Fleisher LA, Fleischmann KE, Auerbach AD, Barnason SA, Beckman JA, Bozkurt B, Davila-Roman VG, Gerhard-Herman MD, Holly TA, Kane GC, Marine JE, Nelson MT, Spencer CC, Thompson A, Ting HH, Uretsky BF, Wijeysundera DN, American College of Cardiology, American Heart Association. 2014 ACC/ AHA guideline on perioperative cardiovascular evaluation and management of patients undergoing noncardiac surgery: a report of the American College of Cardiology/American Heart Association Task Force on practice guidelines. J Am Coll Cardiol 2014;64:e77-137.

208. Siller-Matula JM, Petre A, Delle-Karth G, Huber K, Ay C, Lordkipanidze M, De Caterina R, Kolh P, Mahla E, Gersh BJ. Impact of preoperative use of P2Y12 receptor inhibitors on clinical outcomes in cardiac and non-cardiac surgery: A systematic review and meta-analysis. Eur Heart J Acute Cardiovasc Care 2015; DOl: https://doi.org/10.1177/2048872615585516.

209. Glance LG, Lustik SJ, Hannan EL, Osler TM, Mukamel DB, Qian F, Dick AW. The Surgical Mortality Probability Model: derivation and validation of a simple risk prediction rule for noncardiac surgery. Ann Surg 2012;255:696-702.

210. Rossini R, Musumeci G, Visconti LO, Bramucci E, Castiglioni B, De Servi S, Lettieri C, Lettino M, Piccaluga E, Savonitto S, Trabattoni D, Capodanno D, Buffoli F, Parolari A, Dionigi G, Boni L, Biglioli F, Valdatta L, Droghetti A, Bozzani A, Setacci C, Ravelli P, Crescini C, Staurenghi G, Scarone P, Francetti L, D'Angelo F, Gadda F, Comel A, Salvi L, Lorini L, Antonelli M, Bovenzi F, Cremonesi A, Angiolillo DJ, Guagliumi G, Italian Society of Invasive Cardiology, Italian Association of Hospital Cardiologists, Italian Society for Cardiac Surgery, Italian Society of Vascular and Endovascular Surgery, Italian Association of Hospital Surgeons, Italian Society of Surgery, Italian Society of Anaesthesia and Intensive Care Medicine, Lombard Society of Surgery, Italian Society of Maxillofacial Surgery, Italian Society of Reconstructive Plastic Surgery and Aesthetics, Italian Society of Thoracic Surgeons, Italian Society of Urology, Italian Society of Orthopaedics and Traumatology, Italian Society of Periodontology, Italian Federation of Scientific Societies of Digestive System Diseases Lombardia, Association of Obstetricians Gynaecologists Italian Hospital Lombardia, Society of Ophthalmology Lombardia. Perioperative management of antiplatelet therapy in patients with coronary stents undergoing cardiac and non-cardiac surgery: a consensus document from Italian cardiological, surgical and anaesthesiological societies. Eurolntervention 2014; 10:38-46.

211. Chapman TW, Bowley DM, Lambert AW, Walker AJ, Ashley SA, Wilkins DC. Haemorrhage associated with combined clopidogrel and aspirin therapy. Eur J Vasc Endovasc Surg 2001;22:478-479.

212. Ernst A, Eberhardt R, Wahidi M, Becker HD, Herth FJ. Effect of routine clopidogrel use on bleeding complications after transbronchial biopsy in humans. Chest 2006;129:734-737.

213. Moore M, Power M. Perioperative hemorrhage and combined clopidogrel and aspirin therapy. Anesthesiology 2004;101:792-794.

214. Burger W, Chemnitius JM, Kneissl GD, Rucker G. Low-dose aspirin for secondary cardiovascular prevention - cardiovascular risks after its perioperative withdrawal versus bleeding risks with its continuation - review and meta-analysis. J Intern Med 2005;257:399-414

215. Merritt JC, Bhatt DL. The efficacy and safety of perioperative antiplatelet therapy. J Thromb Thrombolysis 2004;17:21-27.

216. Berger PB, Kleiman NS, Pencina MJ, Hsieh WH, Steinhubl SR, Jeremias A, Sonel A, Browne K, Barseness G, Cohen DJ. Frequency of major noncardiac surgery and subsequent adverse events in the year after drug-eluting stent placement results from the EVENT (Evaluation of Drug-Eluting Stents and Ischemic Events) Registry. JACC Cardiovasc Interv 2010;3:920-927.

217. van Kuijk JP, Flu WJ, Schouten O, Hoeks SE, Schenkeveld L, de Jaegere PP, Bax JJ, van Domburg RT, Serruys PW, Poldermans D. Timing of noncardiac surgery after coronary artery stenting with bare metal or drug-eluting stents. Am J Cardiol 2009:104:1229-1234.

218. Diamantis T, Tsiminikakis N, Skordylaki A, Samiotaki F, Vernadakis S, Bongiorni C, Tsagarakis N, Marikakis F, Bramis I, Bastounis E. Alterations of hemostasis after laparoscopic and open surgery. Hematology 2007;12:561-570.

219. Rajagopalan S, Ford I, Bachoo P, Hillis GS, Croal B, Greaves M, Brittenden J. Platelet activation, myocardial ischemic events and postoperative non-response to aspirin in patients undergoing major vascular surgery. J Thromb Haemost 2007; 5:2028-2035.

220. Grines CL, Bonow RO, Casey DE Jr, Gardner TJ, Lockhart PB, Moliterno DJ, O'Gara P, Whitlow P. Prevention of premature discontinuation of dual antiplatelet therapy in patients with coronary artery stents: a science advisory from the American Heart Association, American College of Cardiology, Society for Cardiovascular Angiography and Interventions, American College of Surgeons, and American Dental Association, with representation from the American College of Physicians. Circulation 2007;115:813-818.
221. Levine GN, Bates ER, Blankenship JC, Bailey SR, Bittl JA, Cercek B, Chambers CE, Ellis SG, Guyton RA, Hollenberg SM, Khot UN, Lange RA, Mauri L, Mehran R, Moussa ID, Mukherjee D, Nallamothu BK, Ting HH, American College of Cardiology Foundation, American Heart Association Task Force on Practice Guidelines, Society for Cardiovascular Angiography and Interventions. 2011 ACCF/AHA/SCAI Guideline for Percutaneous Coronary Intervention. A report of the American College of Cardiology Foundation/American Heart Association Task Force on Practice Guidelines and the Society for Cardiovascular Angiography and Interventions. J Am Coll Cardiol 2011;58:e44-122.

222. Fleisher LA, Beckman JA, Brown KA, Calkins H, Chaikof EL, Fleischmann KE, Freeman WK, Froehlich JB, Kasper EK, Kersten JR, Riegel B, Robb JF, Smith SC Jr, Jacobs AK, Adams CD, Anderson JL, Antman EM, Buller CE, Creager MA, Ettinger SM, Faxon DP, Fuster V, Halperin JL, Hiratzka LF, Hunt SA, Lytle BW, Nishimura R, Ornato JP, Page RL, Riegel B, Tarkington LG, Yancy CW. ACC/ AHA 2007 guidelines on perioperative cardiovascular evaluation and care for noncardiac surgery: a report of the American College of Cardiology/American Heart Association Task Force on Practice Guidelines (Writing Committee to Revise the 2002 Guidelines on Perioperative Cardiovascular Evaluation for Noncardiac Surgery) developed in collaboration with the American Society of Echocardiography, American Society of Nuclear Cardiology, Heart Rhythm Society, Society of Cardiovascular Anesthesiologists, Society for Cardiovascular Angiography and Interventions, Society for Vascular Medicine and Biology, and Society for Vascular Surgery. J Am Coll Cardiol 2007;50:e159-241.

223. Brar SS, Kim J, Brar SK, Zadegan R, Ree M, Liu IL, Mansukhani P, Aharonian V, Hyett R, Shen AY. Long-term outcomes by clopidogrel duration and stent type in a diabetic population with de novo coronary artery lesions. J Am Coll Cardiol 2008;51:2220-2227.

224. Eisenstein EL, Anstrom KJ, Kong DF, Shaw LK, Tuttle RH, Mark DB, Kramer JM, Harrington RA, Matchar DB, Kandzari DE, Peterson ED, Schulman KA, Califf RM. Clopidogrel use and long-term clinical outcomes after drug-eluting stent implantation. JAMA 2007;297:159-168.

225. Navarese EP, Tandjung K, Claessen B, Andreotti F, Kowalewski M, Kandzari DE, Kereiakes DJ, Waksman R, Mauri L, Meredith IT, Finn AV, Kim HS, Kubica J, Suryapranata H, Aprami TM, Di Pasquale G, von Birgelen C, Kedhi E. Safety and efficacy outcomes of first and second generation durable polymer drug eluting stents and biodegradable polymer biolimus eluting stents in clinical practice: comprehensive network meta-analysis. BMJ 2013;347:f6530.

226. Egholm G, Kristensen SD, Thim T, Olesen KK, Madsen M, Jensen SE, Jensen LO, Sorensen HT, Botker HE, Maeng M. Risk associated with surgery within 12 months after coronary drug-eluting stent implantation. J Am Coll Cardiol 2016;68:2622-2632

227. Holcomb CN, Graham LA, Richman JS, Itani KM, Maddox TM, Hawn MT. The incremental risk of coronary stents on postoperative adverse events: a matched cohort study. Ann Surg 2016;263:924-930.

228. Kolh P, Windecker S, Alfonso F, Collet JP, Cremer J, Falk V, Filippatos G, Hamm C, Head SJ, Juni P, Kappetein AP, Kastrati A, Knuuti J, Landmesser U, Laufer G, Neumann FJ, Richter DJ, Schauerte P, Sousa Uva M, Stefanini GG, Taggart DP, Torracca L, Valgimigli M, Wijns W, Witkowski A. 2014 ESC/ EACTS Guidelines on myocardial revascularization: the Task Force on Myocardial Revascularization of the European Society of Cardiology (ESC) and the European Association for Cardio-Thoracic Surgery (EACTS). Developed with the special contribution of the European Association of Percutaneous Cardiovascular Interventions (EAPCI). Eur J Cardiothorac Surg 2014;46:517-592.

229. Savonitto S, D'Urbano M, Caracciolo M, Barlocco F, Mariani G, Nichelatti M, Klugmann S, De Servi S. Urgent surgery in patients with a recently implanted coronary drug-eluting stent: a phase II study of 'bridging' antiplatelet therapy with tirofiban during temporary withdrawal of clopidogrel. BrJ Anaesth 2010;104:285-291.

230. Qamar A, Bhatt DL. Current status of data on cangrelor. Pharmacol Ther 2016;159:102-109.

231. Angiolillo DJ, Firstenberg MS, Price MJ, Tummala PE, Hutyra M, Welsby IJ, Voeltz MD, Chandna H, Ramaiah C, Brtko M, Cannon L, Dyke C, Liu T, Montalescot G, Manoukian SV, Prats J, Topol EJ, BRIDGE Investigators. Bridging antiplatelet therapy with cangrelor in patients undergoing cardiac surgery: a randomized controlled trial. JAMA 2012;307:265-274.

232. Abualsaud AO, Eisenberg MJ. Perioperative management of patients with drugeluting stents. JACC Cardiovasc Interv 2010;3:131-142.

233. Dimitrova G, Tulman DB, Bergese SD. Perioperative management of antiplatelet therapy in patients with drug-eluting stents. HSR Proc Intensive Care Cardiovasc Anesth 2012;4:153-167.

234. Kaluza GL, Joseph J, Lee JR, Raizner ME, Raizner AE. Catastrophic outcomes of noncardiac surgery soon after coronary stenting. J Am Coll Cardiol 2000;35: 1288-1294.

235. van Werkum JW, Heestermans AA, Zomer AC, Kelder JC, Suttorp MJ, Rensing BJ, Koolen JJ, Brueren BR, Dambrink JH, Hautvast RW, Verheugt FW, ten Berg JM. Predictors of coronary stent thrombosis: the Dutch Stent Thrombosis Registry. J Am Coll Cardiol 2009;53:1399-1409. 
236. lakovou I, Schmidt T, Bonizzoni E, Ge L, Sangiorgi GM, Stankovic G, Airoldi F, Chieffo A, Montorfano M, Carlino M, Michev I, Corvaja N, Briguori C, Gerckens U, Grube E, Colombo A. Incidence, predictors, and outcome of thrombosis after successful implantation of drug-eluting stents. JAMA 2005; 293:2126-2130.

237. Bay C, Cyr PL, Jensen I. Estimating The value of cangrelor from eliminating preloading in coronary artery bypass graft (CABG) patients. Value Health 2014; 17:A480.

238. De Servi S, Morici N, Boschetti E, Rossini R, Martina P, Musumeci G, D'Urbano M, Lazzari L, La Vecchia C, Senni M, Klugmann S, Savonitto S. Bridge therapy or standard treatment for urgent surgery after coronary stent implantation: analysis of 314 patients. Vascul Pharmacol 2016;80:85-90.

239. Voeltz MD, Manoukian SV. Cangrelor in patients undergoing cardiac surgery: the BRIDGE study. Expert Rev Cardiovasc Ther 2013;11:811-816.

240. Sawaya FJ, Morice MC, Spaziano M, Mehran R, Didier R, Roy A, Valgimigli M, Kim HS, Woo Park K, Hong MK, Kim BK, Jang Y, Feres F, Abizaid A, Costa RA, Colombo A, Chieffo A, Giustino G, Stone GW, Bhatt DL, Palmerini T, Gilard $M$. Short-versus long-term dual antiplatelet therapy after drug-eluting stent implantation in women versus men: A sex-specific patient-level pooled-analysis of six randomized trials. Catheter Cardiovasc Interv 2017;89:178-189.

241. Gargiulo G, Ariotti S, Santucci A, Piccolo R, Baldo A, Franzone A, Magnani G, Marino M, Esposito G, Windecker S, Valgimigli M. Impact of sex on 2-year clinical outcomes in patients treated with 6-month or 24-month dual-antiplatelet therapy duration: a pre-specified analysis from the PRODIGY trial. JACC Cardiovasc Interv 2016;9:1780-1789.

242. Meredith IT, Tanguay JF, Kereiakes DJ, Cutlip DE, Yeh RW, Garratt KN, Lee DP, Steg PG, Weaver WD, Holmes DR Jr, Brindis RG, Trebacz J, Massaro JM, Hsieh WH, Mauri L; DAPT Study Investigators. Diabetes mellitus and prevention of late myocardial infarction after coronary stenting in the Randomized Dual Antiplatelet Therapy study. Circulation 2016;133:1772-1782.

243. Steg PG, Bhatt DL, Wilson PW, D'Agostino R, Sr., Ohman EM, Rother J, Liau CS, Hirsch AT, Mas JL, Ikeda Y, Pencina MJ, Goto S; REACH Registry Investigators. One-year cardiovascular event rates in outpatients with atherothrombosis. JAMA 2007;297:1197-1206.
244. Cacoub PP, Bhatt DL, Steg PG, Topol EJ, Creager MA; CHARISMA Investigators. Patients with peripheral arterial disease in the CHARISMA trial. Eur Heart J 2009;30:192-201.

245. Bonaca MP, Gutierrez JA, Creager MA, Scirica BM, Olin J. Murphy SA, Braunwald E, Morrow DA. Acute limb ischemia and outcomes with vorapaxar in patients with peripheral artery disease: results from the Trial to Assess the Effects of Vorapaxar in Preventing Heart Attack and Stroke in Patients With Atherosclerosis-Thrombolysis in Myocardial Infarction 50 (TRA2 P-TIMI 50). Circulation 2016;133:997-1005.

246. Franzone A, Piccolo R, Gargiulo G, Ariotti S, Marino M, Santucci A, Baldo A, Magnani G, Moschovitis A, Windecker S, Valgimigli M. Prolonged vs short duration of dual antiplatelet therapy after percutaneous coronary intervention in patients with or without peripheral arterial disease: a subgroup analysis of the PRODIGY randomized clinical trial. JAMA Cardiol 2016;1:795-803.

247. Giustino G, Chieffo A, Palmerini T, Valgimigli M, Feres F, Abizaid A, Costa RA, Hong MK, Kim BK, Jang Y, Kim HS, Park KW, Gilard M, Morice MC, Sawaya F, Sardella G, Genereux P, Redfors B, Leon MB, Bhatt DL, Stone GW, Colombo A. Efficacy and safety of dual antiplatelet therapy after complex PCl. J Am Coll Cardiol 2016;68:1851-1864.

248. Armstrong EJ, Sab S, Singh GD, Lim W, Yeo KK, Waldo SW, Patel M, Reeves R, MacGregor JS, Low RI, Shunk KA, Mahmud E, Rogers JH. Predictors and outcomes of recurrent stent thrombosis: results from a multicenter registry. JACC Cardiovasc Interv 2014;7:1105-1113.

249. Halvorsen S, Storey RF, Rocca B, Sibbing D, Ten Berg J, Grove EL, Weiss TW, Collet JP, Andreotti F, Gulba DC, Lip GY, Husted S, Vilahur G, Morais J, Verheugt FW, Lanas A, Al-Shahi Salman R, Steg PG, Huber K; European Society of Cardiology Working Group on Thrombosis. Management of antithrombotic therapy after bleeding in patients with coronary artery disease and/or atrial fibrillation: expert consensus paper of the European Society of Cardiology Working Group on Thrombosis. Eur Heart J 2016; 38:1455-1462.

250. Amin AP, Bachuwar A, Reid KJ. Chhatriwalla AK, Salisbury AC, Yeh RW, Kosiborod M, Wang TY, Alexander KP, Gosch K, Cohen DJ, Spertus JA, Bach RG. Nuisance bleeding with prolonged dual antiplatelet therapy after acute myocardial infarction and its impact on health status. J Am Coll Cardiol 2013;61:2130-2138. 